\title{
Interventional procrastination strategies for young adults in virtual settings: A systematic review
}

Apr 4, 2021

Joy Xu, ${ }^{1}$ Muntaha Marjia, ${ }^{2}$ Kundan Ahluwalia, ${ }^{1}$ Dana Kukje Zada, ${ }^{1}$ Nazgol Tavakolizadeh, ${ }^{3}$ Margaret Dao ${ }^{1}$

${ }^{1}$ McMaster University, Hamilton, ON, Canada

${ }^{2}$ Ryerson University, Toronto, ON, Canada

${ }^{3}$ University of British Columbia, Vancouver, BC, Canada

Contact Info: joyxu@gmail.com

\begin{abstract}
Background:

The phenomenon of academic procrastination affects nearly $95 \%$ of post-secondary students within academias with its association seen in reduced academic performance. Amidst the onset of COVID-19, this systematic review aims to provide insight towards potential virtual interventions to support personal management of academic procrastination for young adults.
\end{abstract}

\section{Methods:}

The selection procedure followed the Preferred Reporting Items for Systematic Review and MetaAnalyses (PRISMA) guidelines. The protocol was registered with PROSPERO (record ID:

CRD42021234243). Studies were reviewed based on a pre-determined and piloted screening tool with study screening and extraction performed independently among two authors. After reconciliation, disagreements were settled by a third author. Study quality was assessed using the Mixed Methods Appraisal Tool (MMAT) in duplicate. Heterogeneity in study designs, outcomes, and measurements precluded meta and statistical analyses; thus, a qualitative analysis of studies was provided.

\section{Results:}

A total of 49 studies were included with identification of two primary web-based interventions for reducing academic procrastination in a mobile app setting. The first intervention involves emotional management through increasing tolerance and modifying negative emotions which determines the activity with the highest likelihood of procrastination each day, and the subject's resilience or the subject's commitment should be emphasized. The second intervention involves mental imagery for subjects to feel an affinity for his/her future self, and thus decrease procrastination behaviours.

\section{Conclusion:}

Two main virtual interventions were determined from the systematic review: (1) an intervention for managing personal emotions, and (2) an intervention that involves creating a mental image of one's future self. 


\section{KEY WORDS}

Procrastination, young adults, virtual, psychology, interventions, students, college

\section{Background}

Procrastination is a commonly experienced phenomenon characterized as a maladaptive behavioural tendency where the individual may lack effective self-regulation. Consequently, the behavioural outcome resembles an irrational delay or avoidance of certain tasks. As a result, individuals may intentionally avoid responsibilities despite their awareness of potential ensuing consequences.

The underlying cause of procrastination is often theorized to stem from several factors. Firstly, the complex behaviour of procrastination may not merely reflect a lack of motivation or the inability to resist temptations. Rather, procrastination encompasses a lack of acting on intentions [1]. The correlation between procrastination and setting intentions can be seen by a study conducted by Steel et al. where a low measure of intention was observed in a cohort of 152 undergraduate students partaking in an online introductory psychology course. However, a strong correlation was observed between procrastinators and a lack of acting on intentions, which contrasted with non-procrastinators [2]. From an evolutionary lens, procrastinators approach life at a fast pace in comparison to non-procrastinators, which manifests physiologically and psychologically as a result of an individual's environment [3]. Thereby, procrastination is theorized as a beneficial behaviour within unpredictable environments where impulsivity and focus on immediate rewards is necessary. However, these behaviours are no longer consistent with present lifestyles, as self-regulation for future rewards/consequences are favoured. Thus, individuals experiencing habitual chronic procrastination become incompatible with modern day needs in a cycle of additive stress, worsened performance, and greater chances of developing mental or physical illnesses $[3,4]$.

Regardless of the numerous theories determining its cause, procrastination continues to serve as a prominent phenomenon, particularly in young adults within academia [5]. It has been found that approximately 80 - 95\% of post-secondary students have procrastinated in some capacity, while $50 \%$ of these students have experienced detrimental procrastination [6]. Zarick et al. explored the prevalence of procrastination in a sample of 200 students revealing that $95 \%$ of students procrastinate to some degree and $42 \%$ of students procrastinate chronically [5].

Its prevalence also yields academic decline, where these behaviours tend to negatively affect educational achievements in various ways, including lowered grades, withdrawal from courses, and reduced accuracy [5] . Zarick et al. showed that out of the 200 students in the study, one-third and one-fourth of students reported that procrastinative tendencies affected the quality of their assignments and exam results respectively [5].

Although procrastination is evidently prevalent in student populations, there is evidence suggesting that these young adults are willing to change these behaviours. Over half of the student population agreed 
that procrastination requires improvement and should not be a regular aspect of post-secondary education [5]. The prevalence of procrastination is also observed in adult populations where $15-20 \%$ of adults are classified as chronic procrastinators [7].

Despite the prevalence of literature on procrastination prevention strategies, procrastination continues to be an issue affecting productivity and wellbeing. Most interventional studies on procrastination share common challenges in participant compliance due to barriers in intervention designs. These barriers are often attributed to the lack of feasibility, guidance, or usability [8]. For example, interventions requiring attending training sessions or reading material surrounding procrastination tend to have high attrition as individuals feel less motivated and procrastinate on completing these additional tasks. Similarly, group interventions or even one-on-one guided interventions could deter those with heavy workloads, time constraints, social anxiety, and more [8]. Therefore, introducing procrastination intervention through a mobile application can serve as an accessible tool for individuals to take more control over the intervention. The feasibility of this intervention can also be accredited to the prevalence and usability of smartphones, which enable individuals to execute the intervention at their own time and preferred environments. Thus, a virtual intervention may reduce high attrition and dropout rates. Although there has been a dramatic rise in productivity applications, most applications are not rooted in evidence-based approaches nor research appraisal [8].

Therefore, this systematic review aims to assess effective procrastination prevention strategies to be integrated towards virtual interventions, particularly within the development of the Felicity App. The Felicity App is a virtual application targeting individuals aged 15 to 40 years of age to provide productivity enhancement through psychological interventions in various aspects, including procrastination. The systematic review will provide effective interventions for further research to be incorporated as a major feature within the application. This systematic review aims to answer the following research question: What are virtual procrastination prevention strategies that can be implemented into a mobile app setting to promote productive behaviour amongst individuals that are between 15 to 40 years old?

\section{METHODS}

The selection procedure followed the Preferred Reporting Items for Systematic Review and MetaAnalyses (PRISMA) guidelines. The protocol of this systematic review was registered with PROSPERO (record ID: CRD42021234243). Within this systematic review, three databases (Web of Science, PubMed, and ProQuest) were searched from January 4, 2020 to January 17, 2020. Grey implementationfocused literature was searched through the American Psychological Association (APA) webpage. Articles were limited to publication years between January 1995 to December 2020. The eligibility criteria ensured that only studies meeting the following standards were used: (i) Classified as a randomized controlled trial, meta-analysis, systematic reviews, and/or grey literature; (ii) Involved healthy human participants between the ages of 15 to 40; (iii) Included participants of both genders in 
developed countries; (iv) Strategies and concepts with potential for direct application or manipulation in a virtual setting. Using a convergent integrated synthesis approach, a combination of qualitative and quantitative studies were determined. Data screening, quality appraisal, and data extraction was performed by two independent authors followed-by reconciliation and third-author resolution for potential disagreements. Heterogeneity in study designs, outcomes, the scale of measurements, and times precluded meta-analysis. Thereby, a qualitative analysis of included studies was provided using a convergent integrated synthesis approach.

\section{Study design and setting}

This systematic review analyzed the effectiveness of virtual procrastination intervention strategies to determine the relevancy of included studies. A virtual application setting was chosen as the medium for optimal accessibility for the target audience of individuals between the ages of 25 and 40 . According to 2018 data from Statistics Canada, 88.1\% of Canadians from 15 years of age and older owned a smartphone [9]. Furthermore, within this age group, $45.4 \%$ of individuals check their phone every 30 minutes. Cellphones are frequently used amongst the target demographic and subsequently, presenting evidence-based procrastination interventions through a virtual application can enhance accessibility and convenience. Additionally, this application is optimal for adults and adolescents who are employed and/or are students residing in developed countries with greater access to cellphones as they are more likely to become frequent users of the application.

The mixed-method study design was used as qualitative and quantitative information were analyzed through a convergent integrated synthesis approach. Qualitative data provided identification of common characteristics and patterns associated with procrastination such as common habits amongst chronic procrastinators and opinions on various procrastination prevention strategies. Quantitative data presented measurable information for trends and patterns on subjects such as finding a potential correlation between procrastinating on an assignment. Using a mixed method research approach provided all forms of data to be processed, including numerical and observational data. A systematic review allows for a clear identification, selection and analysis of present literature that pertains to the research question being asked and extraction of data that could be implemented into the Felicity App.

\section{Information Sources and Searches}

Based on the eligibility criteria of this study, various forms of literature were reviewed including peerreviewed and grey literature. Searches in databases (Web of Science, PubMed, and ProQuest) were conducted by researchers from January 4, 2020 to January 17, 2020. Studies were limited to the publication period between January 1995 to December 2020. Searches utilized keywords for procrastination, therapy, student, psychology, intervention, and app. To optimize relevance of topics within studies, various keywords were customized for the search (refer to Appendix 1.). 
For grey implementation-focused literature, a search was conducted for documents on the American Psychological Association (APA) website (refer to Appendix 2.). The APA is an American organization consisting of over 122,000 professionals and students within the field of psychology in the United States. The APA works towards the advancement of psychological research, more effective communication of information relating to the field of psychology to the general public and the application of psychology to combat problems within society [10].

Documents found within each grey-implementation literature database were subjected to the same inclusion/exclusion criteria and research question as studies found within Pubmed, Web of Science and ProQuest. Grey literature sources were preferentially selected from government organizations and official organizations to enhance validity.

\section{Eligibility Criteria}

The inclusion and exclusion criteria for this study were predetermined. Studies were only included if written in English, psychology-based, and published between 1995 - 2020. Eligible study designs included randomized controlled trials, meta-analysis, systematic reviews, and grey literature. Studies were deemed suitable if the studied demographic consists of human subjects between the ages of 15 to 
40 that were not diagnosed with any medical complications. Studies consisted of both genders residing in developed countries. Furthermore, studies were only included if strategies and concepts were directly applied or could be manipulated for usage in a virtual setting.

\section{Data Collection Process and Abstraction}

The search results gathered from implementing the search strategy were converted into EndNote (X8) for manual removal of duplicates.

The inclusion and exclusion criteria were used to synthesize questions for the screening tool with the purpose of searching the literature for articles specific to predetermined aims. 25 articles were chosen and individually screened by three authors (MM, NT and KA) during the pilot screening. Modifications to the tool were made with 11 questions to enhance data extraction and reduce bias, where the review of research methods, results, dates, and population were analyzed. The three independent authors reconciled discrepancies and re-modified the screening tool to consolidate objectivity and accuracy of the tool. The finalized screening tool received an accuracy score of $82 \%$.

The modified abstract screening tool included two additional questions from the inclusion/exclusion criteria for enhanced specificity to suit the purpose of this systematic review. The abstract screening tool also adjusted inclusivity to various genders. Similarly, the tool was also modified to exclude literature which target profession-specific studies, as the application is intended to aid a diverse and general population. From various databases, 779 new articles were gathered through collecting related articles and references used in the included articles. 779 new article titles and abstracts were screened by two authors (NT and KA) using the refined screening tool. 
Figure 2. Diagram of screening and study selection process

\section{Identification of Relevant Studies and Data Extraction}

From December 2020 to January 2021, independent authors used Web of Science, Pubmed and ProQuest with keywords to target the specific areas of procrastination. In addition to these databases, the American Psychological Association database was searched as a source of grey literature. From January 20, 2021 to January 25, 2021, with the application of screening tools, two authors (NT and KA) independently screened titles and abstracts of studies where disagreements would be reconciled. Further resolution of disagreements were performed through a third author (MM). The second round of screening took place from January 29, 2021 to January 31, 2021, where included abstracts proceeded for full-text screening by two independent authors (NT and KA), followed by reconciliation and further resolution of disagreements with a third author (MM).The first round of hand search screening where 565 articles went through abstract screening by two authors (NT and KA) between January 31, 2021, and February 7, 2021, produced 40 articles. Between February 7, 2021, and February 14, 2021, Full texts of 40 articles were further screened by the two authors (NT and KA), reconciled, and further disagreements were resolved with the third author (MM). In total, 23 studies were included. Data extracted from these sources included articles titles, study population and location, intervention strategies, implementation of intervention strategies and results. 


\section{Quality Assessment}

Each included study was appraised for quality according to The Mixed Methods Appraisal Tool (MMAT) in order to reduce biases in the research process. The MMAT is a critical appraisal tool created for the appraisal of qualitative, quantitative, and mixed methods-based research based on methodspecific questions. Assessment based on 5-item study design specific questions will assign an overall quality score to each included study. The MMAT will serve as an appropriate measure of quality for included studies in this mixed methods systematic review regarding procrastination. Assessing research quality using the MMAT has been tested and improved for content validity and clarity, increasing confidence and reducing bias in the results of this systematic review. However, more research is needed to determine inter-rater reliability of the MMAT which is significant to allow for reliable appraisal. Using the MMAT to critically appraise various forms of research is essential to ensure interventions introduced in the Felicity App are supported by reliable data.

The quality appraisal process began in February 2021 with two authors (DK and MD) independently appraising and scoring each of the 49 studies based on the MMAT 2018 guide. These scores were then reconciled between the two authors to resolve any disagreements with disagreements resolved by a third author. The reconciled appraisal for each of the 49 studies are outlined in table 1.

\section{Data Extraction}

Following reconciliation, data extraction was performed independently by the two authors (DK and MD) in March 2021. Results from data extraction were reconciled between the two authors to resolve any disagreements. The reconciled data extraction for each of the 49 studies is summarized in table 2.

\section{RESULTS}

\section{Study Characteristics}

A total of 49 articles were included in this systematic review. Of all of the articles, 34 were quantitative, 9 were randomized controlled trials, 5 were mixed methods studies, 1 was a qualitative study, and 1 was a non-randomized study. Furthermore, 7 are from Turkey; 4 are from Sweden; 7 are from the United States; 4 are from Canada; 2 are from Australia, China, and Israel; 12 are from Germany; 1 is from Slovak Republic, Taiwan, Iran, and Ireland. Some studies are from more than one country. All studies passed the initial screening questions and based on the MMAT these studies were rated from a scale of five to one with five being the highest and one being the lowest. Out of the 49 studies, 29 were assigned an overall score of five, 13 were assigned an overall score of four, 5 were assigned an overall score of three, 3 were assigned an overall score of two, and none were assigned a score of one. Characteristics for each category were analyzed to aid in the determination of the appropriate study type, and also provided appropriate determination of articles with procrastination interventions to be added into the Felicity App. 


\section{DISCUSSION}

The systematic review analyzed 48 different articles to understand the psychological underpinnings of procrastination and various interventions that can be implemented for students to reduce academic procrastination. Academic procrastination is set within the context of actions/behaviours which delay academic activities, most commonly (but not limited to) studying for tests, doing homework, and more. This systematic review contributes to the already existing evidence base by extracting information on effective preventative measures for procrastination that have been tested and are supported by evidence. The quantity of relevant strategies combatting procrastination in present literature are limited due to the lack of virtual application or testing of these interventions. Most academic settings are reliant on inperson components, with few academic programs being completely online. From 2004 to 2016, the percent of American undergraduate students taking one or more online undergraduate classes went from $15.6 \%$ to $43.1 \%$ respectively [11]. The COVID-19 pandemic has further exacerbated this transition with online learning, given the requirements for social distancing and regulatory limitation for in-person interaction. With virtual delivery of education due to the COVID-19 pandemic, additional studies are required to fully explore procrastination interventions that can be applied to a virtual setting. In this systematic, many articles discuss potential causes and sources of procrastination rather than identify solutions for unproductive behaviour rooted in procrastination. Due to the heterogeneity of study design, times, participants, and outcomes, a meta-analysis on outcome measures was precluded. In Balkis et al.'s 2017 study Gender differences in the relationship between academic procrastination, satisfaction with academic life and academic performance, it was determined that male students are more vulnerable to the destructive effects of academic procrastination, both regarding academic performance and academic life satisfaction [12]. Ozer et al.'s 2020 study Exploring academic procrastination among Turkish students: Possible gender differences in prevalence and reasons and Özer et al.'s 2005 study Academic procrastination: Prevalence, self-reported reasons, gender difference and it's relation with academic achievement also corroborated this finding [13,14] . Balkis et al.'s 2009 study Prevalence of academic procrastination behavior among pre-service teachers, and its relation with demographics and individual preferences found that in pre-service teachers, there was a high degree of procrastination behaviour at 23\% of teachers [15]. Brownlow et al.'s 2000 study Putting off until tomorrow what is better done today: Academic procrastination as a function of motivation toward college work found that the most important factors that contributed to academic procrastination were perfectionism, low extrinsic motivation, and attribution of one's successes and failures to external factors [16]. Eckert et al.'s 2016 study Overcome procrastination: enhancing emotion regulation skills reduce procrastination found that procrastination involves a difficulty in emotion regulation [17]. This finding was corroborated by Constantin et al.'s 2018 study Anxiety, depression, and procrastination among students: rumination plays a larger mediating role than worry [18]. Häfner et al.'s study Avoiding procrastination through time management: an experimental intervention study found success in using a short-term time management intervention in treating academic procrastination [19]. Haghbin et al.'s 2012 study The Complexity of the Relation between Fear of Failure and Procrastination found that perceived 
competence has an effect on failing and procrastination [20]. Chun Chu et al.'s 2005 study Rethinking Procrastination: Positive Effects of "Active" Procrastination Behavior on Attitudes and Performance was able to identify 2 distinct types of procrastinators: active and passive procrastinators [21]. Similarly, Grunschel et al.'s 2013 study Exploring different types of academic delayers: A latent profile analysis found that there were 4 different types of procrastinators (instead of 2) [22]. Zacks et al.'s 2018 study Academic interventions for academic procrastination: A review of the literature found that instructional intervention from a teaching team is very effective in stopping academic procrastination, but more research is needed to determine individual interventions [23]. Svartdal et al.'s 2020 study How Study Environments Foster Academic Procrastination: Overview and Recommendations also looked at instructional interventions [24]. Kandemir et al.'s 2014 study Reasons of academic procrastination: selfregulation, academic self-efficacy, life satisfaction and demographics variables found that setting goals for the future served an important factor for motivating students [25]. Aznar-Díaz et al.'s 2020 study Mexican and Spanish university students' Internet addiction and academic procrastination: Correlation and potential factors found that increased internet usage correlated with increased academic procrastination [26]. Balkis et. al's 2012 study Analysis of the relation between academic procrastination, academic rational/irrational beliefs, time preferences to study for exams, and academic achievement: a structural model found that having rational beliefs about studying decreased the likelihood of academic procrastination [27]. Blouin $\square$ Hudon et al.’s 2017 study A mental imagery intervention to increase future self $\square$ continuity and reduce procrastination found that the most effective method of improving procrastination was perceiving one's future self, such as creating mental imagery [28]. Boysan et al's 2017 study Associations between procrastination, personality, perfectionism, selfesteem and locus of control emphasized the value of family support in treating academic procrastination [29]. Johnson et al.'s 1995 study An analysis of the contribution of the five factors of personality to variance in academic procrastination evaluated the role of personality characteristics, and found that procrastination scores were inversely correlated with conscientiousness and significantly correlated with neuroticism [30]. However, Steel et al.'s 2016 study Academic procrastination: Psychological antecedents revisited found that procrastination was correlated strongly with conscientiousness, and not any other personality traits [31].

\section{Emotional Management Process}

A web-based intervention to emphasize managing emotions would be helpful in reducing academic procrastination. This includes increasing toleration to and/or modifying harmful emotions [18]. The practical execution of this intervention would involve identification (by the subject) of the activity with the highest likelihood of procrastination each day, followed by identification of whether the task generates aversive emotions or negative affectivity (e.g. contempt, disgust, guilt, boredom). Aversive emotions are feelings of fear, shame, anger, sadness, or hurt, whereas negative affect refers to negative emotions and behaviours such as sadness, disgust, lethargy, fear, and distress [32,33].

This can be done through questionnaires involving the following questions:

1. At what time today do you feel like you may procrastinate?

2. What is the hardest item on your "To-Do" list today? 
3. What activities do you have to do today that you dread doing?

These questions are created to prime individuals to reflect on personal habits and thought patterns. Questionnaires may also incorporate visual cues to sustain the participation of individuals. For example, simple universal pictures such as emojis may be employed, due to its relatability and every-day usage among young adults. Within virtual settings, questionnaires may serve multiple choice questions to enhance simplicity and retain attention. The placement of multiple choice answers may also be considered, as certain ordering of answers may inadvertently influence decisions as well.

Upon assessing personal awareness, individuals should be prompted with potential next steps of identifying tasks which may elicit either aversive or affective emotions. Throughout the process, individuals should be educated on the theories behind procrastination and potential interventions. By gaining greater understanding of personal emotions and theories of procrastination, individuals may feel inclined to continue long-term pursuit of combating procrastination.

After filling in the questionnaire, the virtual space should provide solutions to suit either aversive or affective emotions. For potential aversive emotions, self-efficacy plays a major role in reminding individuals of personal resilience to overcome challenges. Self-efficacy may be bolstered through reminders of past achievements and personalized encouragement centralized on diligence (rather than intrinsic traits such as intelligence). For example, individuals may feel encouraged by reviewing previous accomplishments during adversity to be reminded of their potential. Individuals may also view analytics to see their present progress as a reminder of their diligence. For potential affective emotions, reminders of intention and commitment should serve as a stimulus for individuals to overcome delaying behaviours. For example, individuals may be encouraged to complete a task once recognizing how quickly/easily it can be done. Similarly, individuals may be reminded of their passion through reviewing long-term goals and future aspirations.

Additionally, emotions should also be modified in the long-term through regular maintenance for emotional control. Examples of these interventions include practicing relaxation exercises to alleviate build-up of negative emotions. Within a virtual setting, guided exercises can support an individual within supportive environments (i.e. ambient music and calming narrative to instruct appropriate techniques).

Upon completing the anticipated task, individuals should evaluate their effectiveness of coping and the particular solution that suited their need. A multiple choice reflection questionnaire with quantitative scales can be completed quickly and collect analytics on their behaviour. These statistics can be used to personalize their development and serve as future encouragement for their progress as well.

\section{Mental Imagery}

Mental imagery is an experience where an individual imagines visual depictions about a certain object, event, or scene. For example, thinking about what one will look like in 5 years is a form of creating 
mental imagery about the future. The practical execution of this intervention could involve designing an audio script that creates a mental representation of a student at a certain point in time (notably, at the end of the semester) [28]. The audio script amy include similar statements:

1. "Imagine yourself in _ months" ( $\_$- $=$the number of months the student is away from the end of the semester).

2. Imagine you're sitting at your final exam desk at this very moment.

3. Imagine you're getting your final grades back for this semester.

Within the virtual setting, these guided scripts could be pre-recorded and played for accessibility of the user according to preferences. The user may also set the average time they begin academic work on the average day in their preferences on the mobile app where notifications may remind users during those times. The mental images may cause the subject to feel an affinity for their future self, and thus decrease procrastination behaviours. One limitation to this approach, however, is that this intervention may only be useful for decreasing negative affect, and may not assist with reducing aversive thoughts. For example, when a subject is bored (which demonstrates negative affect), hearing this audio or reading these scripts may remind users of present goals. However, if the subject has a strong disinclination towards an activity they need to complete, this intervention may not serve as effectively.

\section{Barrier to Effective Procrastination Reduction}

Although these interventions show promise in reducing academic procrastination when applied to a mobile app basis, there may be barriers present that prevent effective procrastination reduction. The greatest barrier may be enforcing adherence to these interventions, especially since they are left to the free will of the subject. This occurs because subjects may avoid using the virtual interventions simply because they may desire procrastination rather than work. Circumstances that may trigger this behaviour include when the subject is feeling unmotivated to learn and would instead prefer to procrastinate which may overcome the initiation of these virtual interventions. For example, subjects may simply choose to not partake in certain interventions after some time. This problem can be addressed by introducing notifications and/or pop-ups to stimulate users to consider personal motivation. While this does not solve the problem (as subjects may simply turn off notification, a feature prevalent in most current smartphones, or subjects may simply turn off the app), the reminder serves to prevent simply forgetting the intervention as opposed to avoiding its usage. Another solution would be to implement a rewards system. This can take the form of a "points" that subjects receive when they successfully overcome the tendency to procrastinate on a specific day. However, there is no effective method to monitor if a subject is telling the truth when claiming that they overcame their tendency to procrastinate on certain days. So, this solution presents the problem that subjects can falsely claim that they overcame their tendency to procrastinate, despite not actually having overcome this tendency, leading to a false accumulation of "points" for individuals too focused on virtual incentives over personal growth. 


\section{Limitations}

This systematic review sought to search the literature for effective procrastination interventions that can be implemented through a mobile application. This systematic review was limited to three databases: Web of Science, ProQuest, and Pubmed, which does not serve to fully reflect all current literature of procrastination interventions that can be implemented in a mobile application targeting young adults. The exclusion of other databases from the search limits the procrastination interventions that were reviewed, thus limiting the designed intervention. Although the target population is young adults (15-40 year olds), the majority of the included studies were limited to student samples, specifically university students. Including a greater portion of student-centred studies in this qualitative review excludes a wide range of the target population. Therefore, the intervention might be biased towards a student population rather than the intended young adult population, offering more effective benefits to a smaller subgroup of the target population. When designing the intervention, only studies with a score above 4 in quality were considered. Further research is required for quantitative approaches, as well as room for reporting publication bias and wider external validity.

Using the MMAT to critically appraise the included studies offered several strengths while limiting the systematic review in certain aspects. Since the MMAT allows for assessment of various study designs, the virtual interventions are not as restricted during study selection, allowing for use of diverse data to design a procrastination intervention. Assessing research quality using the MMAT has been tested and improved for content validity and reliability, increasing confidence and reducing bias in the results of this systematic review. The MMAT also allows for clear communication of the quality of each study by providing a score out of five for each included study. Although there are several benefits to using the MMAT in completing this systematic review, there are important limitations which must be considered. The use of this critical appraisal tool necessitates experience with various forms of research and data collection; the lack of this experience may affect the results yielded using this tool. Another limitation of the MMAT is potential variability in the reported reliability and quality of the tool in critical appraisal, therefore increasing subjectivity of the tool and in effect increasing bias in study results. The assignment and use of overall scores through the MMAT may not always serve informatively where a descriptive study could be more useful in obtaining a holistic understanding of the studies being evaluated.

\section{Conclusion}

This systematic review included 49 studies to determine the most effective methods of reducing academic procrastination via mobile app settings. The two main interventions determined include an intervention to tolerate and/or modify harmful emotions, and an intervention to help create a mental image of one's future self. The former intervention is carried out by identification (by the subject) of the activity with the highest likelihood of procrastination each day, followed by identification of whether the task generates aversive emotions or negative affect, then either a reminder of resilience or an attempt to commit to the task. Additionally, modification of emotions through practicing relaxation exercises should also be performed. The latter intervention is carried out by playing an audio script (or text for reading) that creates a mental representation of a student at the end of the semester, which is to be 
played before the user begins doing academic work each day. Despite identification of the interventions required to reduce academic procrastination, more research needs to be done to determine whether these interventions are efficacious in reducing academic procrastination in a mobile-app setting.

\section{REFERENCES}

[1] Dewitte S, Schouwenburg HC. Procrastination, temptations, and incentives: The struggle between the present and the future in procrastinators and the punctual. Eur J Pers. 2002; https://doi.org/10.1002/per.461

[2] Sirois FM, van Eerde W, Argiropoulou MI. Is procrastination related to sleep quality? Testing an application of the procrastination-health model. Cogent Psychol. 2015; https://doi.org/10.1080/23311908.2015.1074776

[3] Svartdal F, Granmo S, Færevaag FS. On the Behavioral Side of Procrastination: Exploring Behavioral Delay in Real-Life Settings. Front Psychol. 2018; https://doi.org/10.3389/fpsyg.2018.00746

[4] Chen B, Chang L. Procrastination as a Fast Life History Strategy. J Evol Psychol. 2016; https://doi.org/10.1177/1474704916630314

[5] Kim KR, Seo EH. The relationship between procrastination and academic performance: A meta-analysis. Pers Individ Differ. 2015; https://doi.org/10.1016/j.paid.2015.02.038

[6] Taura A. Towards understanding procrastination. ATBU Journal of Science, Technology And Education. 2017.

[7] Steel P. The Nature of Procrastination: A Meta-Analytic and Theoretical Review of Quintessential Self-Regulatory Failure. Psychol Bull. 2007; https://doi.org/10.1037/0033-2909.133.1.65

[8] van Eerde W, Klingsieck KB. Overcoming procrastination? A meta-analysis of intervention studies. Rev Educ Res. 2018;

https://doi.org/10.1016/j.edurev.2018.09.002

[9] Canada S. Smartphone use and smartphone habits by gender and age group. Can J Stat. 2018; https://doi.org/10.25318/2210011501-eng 
[10] American Psychological Association. About APA. In: About APA. American Psychological Association. 2020. https://www.apa.org/about. Accessed 03 Apr 2021.

[11] Snyder TD, de Brey C, Dillow SA. Digest of Education Statistics 2018. NCES. 2019.

[12] Balkis M, Erdinç D. Gender Differences in the Relationship between Academic Procrastination, Satifaction with Academic Life and Academic Performance. Rev. electrón. investig. psicoeduc. psicopedag. 2017;

https://doi.org/10.14204/ejrep.41.16042

[13] Özer BU, Demir A, Ferrari JR. Exploring Academic Procrastination Among Turkish Students: Possible Gender Differences in Prevalence and Reasons. Eur J Soc Psychol. 2009; https://doi.org/10.3200/SOCP.149.2.241-257

[14] Özer BU. ACADEMIC PROCRASTINATION: PREVALENCE, SELF-REPORTED REASONS, GENDER DIFFERENCE AND IT'S RELATION WITH ACADEMIC ACHIEVEMENT. In: ACADEMIC PROCRASTINATION: PREVALENCE, SELFREPORTED REASONS, GENDER DIFFERENCE AND IT'S RELATION WITH ACADEMIC ACHIEVEMENT. The Graduate School of Social Sciences of Middle East Technical University. 2005. https://open.metu.edu.tr/bitstream/handle/11511/15238/index.pdf?sequence=1. Accessed $03 \mathrm{Apr}$ 2021.

[15] Balkis M, Erdinç D. Prevalence of academic procrastination behavior among pre-service teachers, and its relationship with demographics and individual preferences. Journal of Theory and Practice in Education. 2009.

[16] Brownlow S, Reasinger RD. Putting off Until Tomorrow What is Better Done Today: Academic Procrastination as a Function of Motivation Toward College Work. J Pers Soc Psychol. 2000.

[17] Eckert M, Ebert DD, Lehr D, Sieland B, Berking M. Overcome procrastination: Enhancing emotion regulation skills reduce procrastination. Learn Individ Differ. 2016; https://doi.org/10.1016/j.lindif.2016.10.001

[18] Constantin K, English MM, Mazmanian D. Anxiety, Depression, and Procrastination Among Students: Rumination Plays a Larger Mediating Role than Worry. J Ration Emot Cogn Behav Ther. 2018; https://doi.org/10.1007/s10942-017-0271-5 
[19] Häfner A, Oberst V, Stock A. Avoiding procrastination through time management: an experimental intervention study. Educ Stud. 2014;

https://doi.org/10.1080/03055698.2014.899487

[20]Haghbin M, McCaffrey A, Pychyl TA. The Complexity of the Relation between Fear of Failure and Procrastination. J Ration Emot Cogn Behav Ther. 2012;

https://doi.org/10.1007/s10942-012-0153-9

[21] Chun Chu AH, Choi JN. Rethinking Procrastination: Positive Effects of "Active" Procrastination Behavior on Attitudes and Performance. J Soc Psychol. 2005; https://doi.org/10.3200/SOCP.145.3.245-264

[22] Grunschel C, Patrzek J, Fries S. Exploring different types of academic delayers: A latent profile analysis. Learn Individ Differ. 2013; https://doi.org/10.1016/j.lindif.2012.09.014

[23] Zacks S, Hen M. Academic interventions for academic procrastination: A review of the literature. J Prev Interv Community. 2018; https://doi.org/10.1080/10852352.2016.1198154

[24] Svartdal F, Dahl TI, Gamst-Klaussen T, Koppenborg M, Klingsieck KB. How Study Environments Foster Academic Procrastination: Overview and Recommendations. Front Psychol. 2020;

https://doi.org/10.3389/fpsyg.2020.540910

[25] Kandemir M. Reasons of Academic Procrastination: Self-regulation, Academic Selfefficacy, Life Satisfaction and Demographics Variables. Procedia Soc Behav Sci. 2014; https://doi.org/10.1016/j.sbspro.2014.09.179

[26] Aznar-Díaz I, Romero-Rodríguez J, García-González A, Ramírez-Montoya M. Mexican and Spanish university students' Internet addiction and academic procrastination: Correlation and potential factors. PloS One. 2020; https://doi.org/10.1371/journal.pone.0233655

[27] Balkis M, Duru E, Bulus M. Analysis of the relation between academic procrastination, academic rational/irrational beliefs, time preferences to study for exams, and academic achievement: a structural model. Eur J Psychol Educ. 2013; https://doi.org/10.1007/s10212-012-0142-5 
[28] Blouin $\square$ Hudon EC, Pychyl TA. A Mental Imagery Intervention to Increase Future Self $\square$ Continuity and Reduce Procrastination. Appl Psychol. 2017;

https://doi.org/10.1111/apps.12088

[29] Boysan M, Kiral E. Associations between procrastination, personality, perfectionism, selfesteem and locus of control. Br J Guid Counc. 2017;

https://doi.org/10.1080/03069885.2016.1213374

[30] Johnson JL, Bloom AM. An analysis of the contribution of the five factors of personality to variance in academic procrastination. Pers Individ Differ.1995;

https://doi.org/10.1016/0191-8869(94)00109-6

[31] Steel P, Klingsieck KB. Academic Procrastination: Psychological Antecedents Revisited. Aust Psychol. 2016;

https://doi.org/10.1111/ap.12173

[32] Saarni C. Coping with Aversive Feelings. Motiv Emot. 1997;

https://doi.org/10.1023/A:1024474314409

[33] Ackerman CE. What is Positive and Negative Affect in Psychology?. In: Positive Emotions. Positive Psychology. 2021. https://positivepsychology.com/positive-negative-affect/. Accessed 03 Apr 2021.

[34] Bytamar JM, Saed O, Khakpoor S. Emotion Regulation Difficulties and Academic Procrastination. Front Psychol. 2020;

https://doi.org/10.3389/fpsyg.2020.524588

[35] Eckert M, Ebert DD, Lehr D, Sieland B, Berking M. Does SMS-Support Make a Difference? Effectiveness of a Two-Week Online-Training to Overcome Procrastination. A Randomized Controlled Trial. Frontiers Psychol 2018;

https://doi.org/10.3389/fpsyg.2018.01103

[36] Eisenbeck N, Carreno DF, Uclés-Juárez R. From psychological distress to academic procrastination: Exploring the role of psychological inflexibility. J Contextual Behav Sci. 2019; https://doi.org/10.1016/j.jcbs.2019.07.007

[37] Ferrari JR, Emmons RA. Methods of Procrastination and their Relation to Self-Control and Self-Reinforcement: An Exploratory Study. Soc Behav Pers. 1995. 
[38] Fritzsche BA, Rapp Young B, Hickson KC. Individual differences in academic procrastination tendency and writing success. Pers Individ Differ. 2003;

https://doi.org/10.1016/S0191-8869(02)00369-0

[39] Glick DM, Millstein DJ, Orsillo SM. A preliminary investigation of the role of psychological inflexibility in academic procrastination. J Contextual Behav Sci. 2014; https://doi.org/10.1016/j.jcbs.2014.04.002

[40] Grunschel C, Patrzek J, Fries S. Exploring reasons and consequences of academic procrastination: an interview study. Eur J Psychol Educ. 2013;

https://doi.org/10.1007/s10212-012-0143-4

[41] Grunschel C, Patrzek J, Klingsieck KB, Fries S. "I'll stop procrastinating now!" Fostering specific processes of self-regulated learning to reduce academic procrastination. J Prev Interv Community. 2018;

https://doi.org/10.1080/10852352.2016.1198166

[42] Jackson T, Fritch A, Nagasaka T, Pope L. Procrastination and perceptions of past, present, and future. Individ Differ Res. 2003.

[43] Jin H, Wang W, Lan X. Peer Attachment and Academic Procrastination in Chinese College Students: A Moderated Mediation Model of Future Time Perspective and Grit. Front Psychol. 2019;

https://doi.org/10.3389/fpsyg.2019.02645

[44] Job V, Walton GM, Bernecker K, Dweck CS. Implicit Theories About Willpower Predict Self-Regulation and Grades in Everyday Life. J Pers Soc Psychol. 2015; https://doi.org/10.1037/pspp0000014

[45]Krispenz A, Gort C, Schültke L, Dickhäuser O. How to Reduce Test Anxiety and Academic Procrastination Through Inquiry of Cognitive Appraisals: A Pilot Study Investigating the Role of Academic Self-Efficacy. Front Psychol. 2019;

https://doi.org/10.3389/fpsyg.2019.01917

[46] Küchler A, Albus P, Ebert DD, Baumeister H. Effectiveness of an internet-based intervention for procrastination in college students (StudiCare Procrastination): Study protocol of a randomized controlled trial. Internet Interv. 2019;

https://doi.org/10.1016/j.invent.2019.100245 
[47] Loeffler SN, Stumpp J, Grund S, Limberger MF, Ebner-Priemer UW. Fostering selfregulation to overcome academic procrastination using interactive ambulatory assessment.Learn Individ Differ. 2019; https://doi.org/10.1016/j.lindif.2019.101760

[48] Malouff JM, Schutte NS. The Efficacy of Interventions Aimed at Reducing Procrastination: A Meta $\square$ Analysis of Randomized Controlled Trials. J Couns Dev. 2019;

https://doi.org/10.1002/jcad.12243

[49] Martincekova L, Enright RD. The effects of self-forgiveness and shame-proneness on procrastination: exploring the mediating role of affect. Curr Psychol. 2020; https://doi.org/10.1007/s12144-018-9926-3

[50] Milgram N, Marshevsky S, Sadeh C. Correlates of Academic Procrastination: Discomfort, Task Aversiveness, and Task Capability.J Psychol. 1995; https://doi.org/10.1080/00223980.1995.9914954

[51] Rozental A, Forsell E, Svensson A, Forsström D, Andersson G, Carlbring P. Differentiating Procrastinators from Each Other: A Cluster Analysis.Cogn Behav Ther. 2015; https://doi.org/10.1080/16506073.2015.1059353

[52] Rozental A, Forsström D, Lindner P, et al. Treating Procrastination Using Cognitive Behavior Therapy: A Pragmatic Randomized Controlled Trial Comparing Treatment Delivered via the Internet or in Groups.Behav Ther. 2018;

https://doi.org/10.1016/j.beth.2017.08.002

[53] Vodanovich SJ, Seib HM. Relationship between Time Structure and Procrastination. Psychological reports. 1997;

https://doi.org/10.2466/pr0.1997.80.1.211

[54] Wessel J, Bradley GL, Hood M. A low $\square$ intensity, high $\square$ frequency intervention to reduce procrastination. Appl Psychol. 2020;

https://doi.org/10.1111/apps.12293

[55] Yile D. Characteristics and intervention of academic procrastination of students-An analysis based on 1192 effective samples of Huaqiao University. Int J Electr Eng Educ. 2020; https://doi.org/10.1177/0020720920930356 


\section{DECLARATIONS}

Funding: This systematic review was funded in part by a grant from The Duke of Edinburgh's International Award through the P2P program and in partnership with the federal government of Canada.

\section{Conflicts of interest/Competing interests:}

The authors declared no potential conflicts of interest with respect to the research, authorship, and/or publication of this article.

\section{Availability of data and material:}

\section{Code availability:}

\section{N/A}

\section{Authors' contributions:}

JX devised and supervised the project and secured funding acquisition. MM, KA, DZ, NT and MD wrote the manuscript. MM and NT created figures. DZ, NT and MD computed numerical data and created tables. KA and NT performed the article screening. DZ and MD carried out the quality assessment. All authors refined and approved the manuscript.

\section{Database Searches}

\section{Appendix 1}

\begin{tabular}{|l|l|l|}
\hline Database & Search & Results \\
\hline Web of Science & $\begin{array}{l}\text { (Procrastinat*) AND (psych*) } \\
\text { AND (student*) AND } \\
\text { (interven*) }\end{array}$ & 81 \\
\hline ProQuest & $\begin{array}{l}\text { (Procrastinat*) AND (stud*) } \\
\text { AND (psych*) AND (interven*) } \\
\text { AND (app*) }\end{array}$ & 77 \\
\hline PubMed & $\begin{array}{l}\text { (Procrastinat*) AND (stud*) } \\
\text { AND (psych*) }\end{array}$ & 175 \\
\hline
\end{tabular}

\section{Appendix 2}

\begin{tabular}{|l|l|l|}
\hline Database & Search & Results \\
\hline
\end{tabular}




\begin{tabular}{|l|l|l|}
\hline $\begin{array}{l}\text { American Psychological } \\
\text { Association }\end{array}$ & $\begin{array}{l}\text { (Procrastinat*) AND (stud*) } \\
\text { AND (psych*) AND (education) } \\
\text { AND (web page) }\end{array}$ & 48 \\
\hline
\end{tabular}

Table 1:

\begin{tabular}{|c|c|c|c|c|c|c|c|c|c|c|c|c|c|c|c|c|c|c|c|c|c|c|c|c|}
\hline & 1.1 & 1.2 & $|1.3|$ & 1.4 & 1.52 & 2.12 & \begin{tabular}{l|l}
2.2 & 2
\end{tabular} & \begin{tabular}{l|l}
2.3 & 2
\end{tabular} & $2.4 \mid 2$ & \begin{tabular}{l|l}
2.5 & 3
\end{tabular} & \begin{tabular}{l|l}
3.1 & 3.
\end{tabular} & \begin{tabular}{l|l}
3.2 & 3
\end{tabular} & \begin{tabular}{l|l|l}
3.3 & 3
\end{tabular} & \begin{tabular}{l|l}
3.4 & 3
\end{tabular} & 3.5 & 4.1 & 4.2 & 4.3 & 4.4 & 4.5 & 5.1 & 5.2 & 5.3 & \begin{tabular}{|l|l}
5.4 & 5
\end{tabular} \\
\hline $\begin{array}{l}\text { Balkis M, } \\
2017\end{array}$ & & & & & & & & & & & & & & & & * & * & * & * & * & & & & \\
\hline $\begin{array}{l}\text { Balkis M, } \\
2009\end{array}$ & & & & & & & & & & & & & & & & * & * & * & * & * & & & & \\
\hline $\begin{array}{l}\text { Brownlow } \\
\text { S, } 2000\end{array}$ & & & & & & & & & & & & & & & & * & * & * & * & * & & & & \\
\hline $\begin{array}{l}\text { Constantin } \\
\mathrm{K}, 2018\end{array}$ & & & & & & & & & & & & & & & & & & * & * & * & & & & \\
\hline $\begin{array}{l}\text { Eckert, } \\
2016\end{array}$ & & & & & & * & * & * & * & * & & & & & & & & & & & & & & \\
\hline $\begin{array}{l}\text { Ozer B, } \\
2009\end{array}$ & & & & & & & & & & & & & & & & * & * & * & * & * & & & & \\
\hline $\begin{array}{l}\text { Ferrari J, } \\
1995\end{array}$ & & & & & & & & & & & & & & & & * & * & * & * & * & & & & \\
\hline $\begin{array}{l}\text { Glick DM, } \\
2013\end{array}$ & & & & & & & & & & & & & & & & * & * & * & * & * & & & & \\
\hline $\begin{array}{l}\text { Hafner A, } \\
2014\end{array}$ & & & & & & & & * & & & & & & & & & & & & & & & & \\
\hline $\begin{array}{l}\text { Haghbin } \\
\text { M, } 2018\end{array}$ & & & & & & & & & & & & & & & & & & * & * & * & & & & \\
\hline $\begin{array}{l}\text { Chun Chu } \\
\text { A, } 2010\end{array}$ & & & & & & & & & & & & & & & & * & * & * & * & * & & & & \\
\hline $\begin{array}{l}\text { Zacks, } \\
2018\end{array}$ & * & * & * & * & & & & & & & & & & & & & & & & & & & & \\
\hline $\begin{array}{l}\text { Svartdal } \\
F, 2020\end{array}$ & & & & & & & & & & & & & & & & & & & & & * & * & * & * \\
\hline $\begin{array}{l}\text { Jackson, } \\
2003\end{array}$ & & & & & & & & & & & & & & & & * & * & * & * & * & & & & \\
\hline $\begin{array}{l}\text { Kandemir, } \\
2014\end{array}$ & & & & & & & & & & & & & & & & & * & * & * & * & & & & \\
\hline $\begin{array}{l}\text { Milgram } \\
\text { N, } 1995\end{array}$ & & & & & & & & & & & & & & & & t & & * & * & * & & & & \\
\hline
\end{tabular}




\begin{tabular}{|c|c|c|c|c|c|c|c|c|c|c|c|c|c|c|c|c|c|c|c|c|}
\hline \begin{tabular}{|l} 
Ozer B, \\
2005
\end{tabular} & & & & & & & & & & & & & & * & * & * & * & * & & \\
\hline $\begin{array}{l}\text { Rozental } \\
\text { A, } 2014\end{array}$ & & & & & & & & & & & & & & & & & & & * & \\
\hline $\begin{array}{l}\text { Webb T, } \\
2010\end{array}$ & & & & & & & & & & & & & & * & * & * & * & * & & \\
\hline $\begin{array}{l}\text { Wohl M, } \\
2010\end{array}$ & & & & & & & & & & & & & & * & & * & * & * & & \\
\hline $\begin{array}{l}\text { Aznar- } \\
\text { Diaz I, } \\
2020\end{array}$ & & & & & & & & & & & & & & * & * & * & * & * & & \\
\hline $\begin{array}{l}\text { Balkis M, } \\
2012\end{array}$ & & & & & & & & & & & & & & * & * & * & * & * & & \\
\hline $\begin{array}{l}\text { Balkis M, } \\
2019\end{array}$ & & & & & & & & & & & & & & * & * & * & * & * & & \\
\hline $\begin{array}{l}\text { Bennett C, } \\
2019\end{array}$ & & & & & & & & & & & & & & * & * & * & * & * & & \\
\hline \begin{tabular}{|l} 
Bloun \\
Houdin, \\
2018
\end{tabular} & & & & & * & & * & * & * & & & & & & & & & & & \\
\hline $\begin{array}{l}\text { Boysan, } \\
2016\end{array}$ & & & & & & & & & & & & & & * & * & * & * & * & & \\
\hline \begin{tabular}{|l} 
Eckert M, \\
2018
\end{tabular} & & & & & & * & & * & & & & & & & & & & & & \\
\hline \begin{tabular}{|l|} 
Elsenbeck \\
N, 2019
\end{tabular} & & & & & & & & & & & & & & * & * & * & * & * & & \\
\hline $\begin{array}{l}\text { Fritzche B, } \\
2003\end{array}$ & & & & & & & & & & & & & & * & * & * & * & * & & \\
\hline $\begin{array}{l}\text { Grund A, } \\
2018\end{array}$ & & & & & & & & & & & & & & * & & * & * & * & & \\
\hline $\begin{array}{l}\text { Grunschel } \\
\text { C, } 2013\end{array}$ & & & & & & & & & & * & & * & * & & & & & & & \\
\hline Jin H, 2019 & & & & & & & & & & & & & & * & * & * & * & * & & \\
\hline $\begin{array}{l}\text { Job V, } \\
2015\end{array}$ & & & & & & & & & & & & & & * & * & * & * & * & & \\
\hline $\begin{array}{l}\text { Johnson J, } \\
1995\end{array}$ & & & & & & & & & & & & & & * & * & * & * & * & & \\
\hline Ko C, 2019 & & & & & & * & & * & * & & & & & * & * & * & & * & & \\
\hline Krispenz A, & & & & & & & & & & & & & & & & & & & & \\
\hline
\end{tabular}




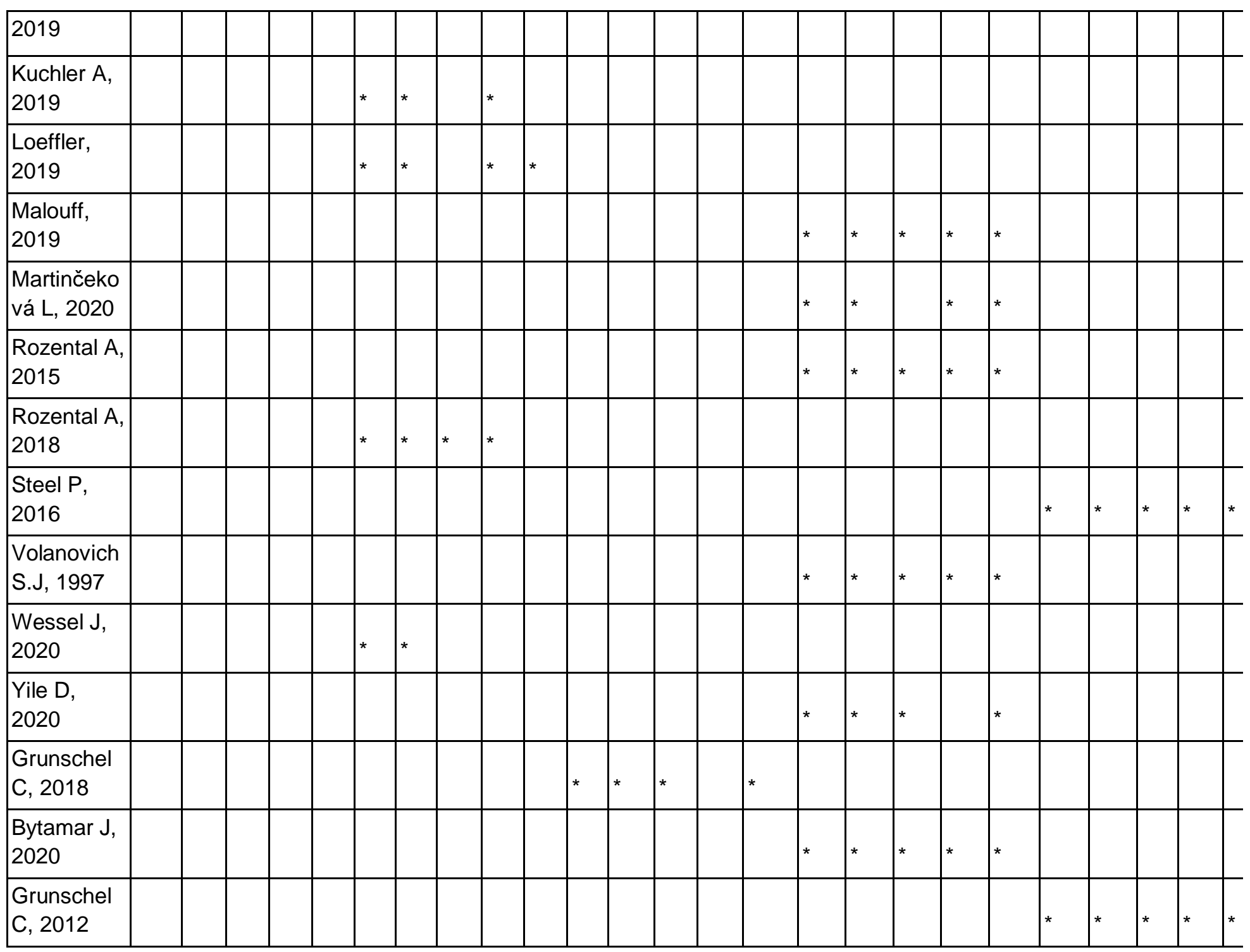

Table 2:

\begin{tabular}{|c|c|c|c|c|c|}
\hline $\begin{array}{l}\text { Study and } \\
\text { Author }\end{array}$ & Country & $\begin{array}{l}\text { Study Design, } \\
\text { Duration and } \\
\text { Participants }\end{array}$ & Type of Data & Outcome & Quality Score \\
\hline $\begin{array}{l}\text { Balkis M.. } \\
\text { Gender } \\
\text { differences in } \\
\text { the } \\
\text { relationship } \\
\text { between } \\
\text { academic }\end{array}$ & Turkey & $\begin{array}{l}\text { Participants: } \\
441 \\
\text { undergraduat } \\
\text { e students } 18- \\
26 \text { years old. } \\
49.4 \% \text { female } \\
\text { and } 50.6 \%\end{array}$ & $\begin{array}{l}\text { Quantitative. } \\
\text { Aitken } \\
\text { procrastinatio } \\
\text { n inventory } \\
\text { (API) } \\
\text { determined } \\
\text { students' level }\end{array}$ & $\begin{array}{l}\text { Male students } \\
\text { are more } \\
\text { vulnerable to } \\
\text { destructive } \\
\text { effects of } \\
\text { academic } \\
\text { procrastinatio }\end{array}$ & $4 / * * * * *$ \\
\hline
\end{tabular}




\begin{tabular}{|c|c|c|c|c|c|}
\hline $\begin{array}{l}\text { procrastinatio } \\
\mathrm{n} \text {, satisfaction } \\
\text { with } \\
\text { academic life } \\
\text { and academic } \\
\text { performance }\end{array}$ & & male. & $\begin{array}{l}\text { of academic } \\
\text { procrastinatio } \\
\mathrm{n} \text { through a } \\
\text { self-report } \mathrm{T} \\
\text { or F } \\
\text { questionnaire, } \\
\text { academic } \\
\text { satisfaction } \\
\text { scale and } \\
\text { demographic } \\
\text { information } \\
\text { form to } \\
\text { determine } \\
\text { age, gender, } \\
\text { and major. } \\
\text { Coefficient of } \\
\text { Pearson } \\
\text { Correlation } \\
\text { was utilized } \\
\text { to set the } \\
\text { relationships } \\
\text { between the } \\
\text { variables }\end{array}$ & $\begin{array}{l}\mathrm{n} \text { in terms of } \\
\text { academic } \\
\text { performance } \\
\text { and academic } \\
\text { life } \\
\text { satisfaction }\end{array}$ & \\
\hline $\begin{array}{l}\text { Balkis, M.. } \\
\text { Prevalence of } \\
\text { academic } \\
\text { procrastinatio } \\
\text { n behavior } \\
\text { among pre- } \\
\text { service } \\
\text { teachers, and } \\
\text { its relation } \\
\text { with } \\
\text { demographics } \\
\text { and } \\
\text { individual } \\
\text { preferences }\end{array}$ & Turkey & $\begin{array}{l}\text { Participants: } \\
580 \text { students } \\
\text { ( } 329 \text { female } \\
\text { and } 251 \\
\text { male) in } \\
\text { different } \\
\text { fields at the } \\
\text { Faculty of } \\
\text { Education in } \\
\text { Pamukkale } \\
\text { University. } \\
\text { Age range } \\
\text { 19-28. API } \\
\text { was designed } \\
\text { to measure } \\
\text { the tendency } \\
\text { of students to } \\
\text { procrastinate } \\
\text { in their } \\
\text { academic } \\
\text { tasks. The } \\
\text { completion }\end{array}$ & $\begin{array}{l}\text { Aitken's } \\
\text { procrastinatio } \\
\mathrm{n} \text { inventory } \\
\text { and personal } \\
\text { information } \\
\text { sheet were } \\
\text { used to gather } \\
\text { data. } \\
\text { Coefficient of } \\
\text { Pearson } \\
\text { Correlation } \\
\text { was utilized } \\
\text { to set the } \\
\text { relationships } \\
\text { between the } \\
\text { variables }\end{array}$ & $\begin{array}{l}23 \% \text { of pre- } \\
\text { service } \\
\text { teachers } \\
\text { exhibited } \\
\text { procrastinatio } \\
\mathrm{n} \text { behavior at } \\
\text { a high level } \\
\text { and } 27 \% \\
\text { experienced } \\
\text { average } \\
\text { levels of } \\
\text { procrastinatio } \\
\text { n. The } \\
\text { average } \\
\text { procrastinatio } \\
\mathrm{n} \text { behavior } \\
\text { scores for } \\
\text { male } \\
\text { prospective } \\
\text { teachers is } \\
\text { higher than } \\
\text { the average }\end{array}$ & $4 / * * * * * *$ \\
\hline
\end{tabular}




\begin{tabular}{|c|c|c|c|}
\hline & . & $\begin{array}{l}\text { lasted } 10-15 \\
\text { minutes. } \\
\text { Turkey test } \\
\text { analysis was } \\
\text { applied }\end{array}$ & $\begin{array}{l}\text { for females. } \\
\text { There is a } \\
\text { negative } \\
\text { correlation } \\
\text { between the } \\
\text { procrastinatio } \\
\text { n behavior } \\
\text { and age of } \\
\text { prospective } \\
\text { teachers. The } \\
\text { average } \\
\text { scores for the } \\
\text { students who } \\
\text { prefer to } \\
\text { study after } \\
\text { 8PM is } \\
\text { higher than } \\
\text { the average of } \\
\text { those who } \\
\text { prefer to } \\
\text { study early } \\
\text { morning, at } \\
\text { noon and the } \\
\text { evening. The } \\
\text { average } \\
\text { scores for the } \\
\text { prospective } \\
\text { students who } \\
\text { prefer to } \\
\text { study a week } \\
\text { before exams } \\
\text { is higher than } \\
\text { the average } \\
\text { scores for } \\
\text { those who } \\
\text { prefer to } \\
\text { study up to } \\
\text { the very day } \\
\text { and two } \\
\text { weeks before } \\
\text { the exam. } \\
\text { There is a } \\
\text { negative } \\
\text { correlation } \\
\text { between } \\
\text { procrastinatio }\end{array}$ \\
\hline
\end{tabular}




\begin{tabular}{|c|c|c|c|c|c|}
\hline & & & & $\begin{array}{l}\mathrm{n} \text { and } \\
\text { academic } \\
\text { achievement } \\
\text { of } \\
\text { prospective } \\
\text { teachers }\end{array}$ & \\
\hline $\begin{array}{l}\text { Brownlow, } \\
\text { S.. (2000). } \\
\text { Putting off } \\
\text { until } \\
\text { tomorrow } \\
\text { what is better } \\
\text { done today: } \\
\text { Academic } \\
\text { procrastinatio } \\
\text { n as a } \\
\text { function of } \\
\text { motivation } \\
\text { toward } \\
\text { college work }\end{array}$ & United States & $\begin{array}{l}\text { Participants: } \\
96 \\
\text { undergraduat } \\
\text { e students ( } 48 \\
\text { men and } 48 \\
\text { women) } \\
\text { Participants } \\
\text { completed the } \\
\text { study alone or } \\
\text { in mixed-sex } \\
\text { groups of 2- } \\
10 \text { people. }\end{array}$ & $\begin{array}{l}\text { The } \\
\text { procrastinatio } \\
n \text { assessment } \\
\text { scale-students } \\
\text { was used to } \\
\text { collect data } \\
\text { on the } \\
\text { frequency of } \\
\text { procrastinatio } \\
\text { n, the reasons } \\
\text { students } \\
\text { procrastinate } \\
\text { and an } \\
\text { indication of } \\
\text { how much } \\
\text { desire exists } \\
\text { to change. } \\
\text { Attributional } \\
\text { style was } \\
\text { measured } \\
\text { through the } \\
\text { multidimensi } \\
\text { onal-multi- } \\
\text { attributional } \\
\text { causality } \\
\text { scale. Fear of } \\
\text { negative } \\
\text { evaluation } \\
\text { was measured } \\
\text { through the } \\
\text { brief fear of } \\
\text { negative } \\
\text { evaluation } \\
\text { scale to } \\
\text { assess } \\
\text { concern and } \\
\text { apprehension } \\
\text { about } \\
\text { negative }\end{array}$ & $\begin{array}{l}\text { Lack of } \\
\text { extrinsic } \\
\text { motivation, } \\
\text { coupled with } \\
\text { perfectionism } \\
\text { and an } \\
\text { external } \\
\text { attributional } \\
\text { style } \\
\text { contributed to } \\
\text { procrastinatio } \\
\text { n. Low- } \\
\text { procrastinatin } \\
\text { g students } \\
\text { were more } \\
\text { motivated by } \\
\text { internal and } \\
\text { external } \\
\text { forces than } \\
\text { were high } \\
\text { procrastinator } \\
\text { s. Moreover, } \\
\text { high } \\
\text { procrastinator } \\
\text { s and those } \\
\text { low in } \\
\text { intrinsic } \\
\text { motivation } \\
\text { made external } \\
\text { attributions } \\
\text { (to context } \\
\text { and luck) for } \\
\text { their } \\
\text { successes, but } \\
\text { students who } \\
\text { were } \\
\text { intrinsically } \\
\text { motivated } \\
\text { took personal }\end{array}$ & $4 / * * * * *$ \\
\hline
\end{tabular}




\begin{tabular}{|c|c|c|c|c|c|}
\hline & & & $\begin{array}{l}\text { evaluations } \\
\text { received by } \\
\text { others }\end{array}$ & $\begin{array}{l}\text { responsibility } \\
\text { for both their } \\
\text { successes } \\
\text { and failures. } \\
\text { These } \\
\text { findings } \\
\text { suggest that } \\
\text { both salient } \\
\text { motivators } \\
\text { and stable } \\
\text { personality } \\
\text { factors } \\
\text { contribute to } \\
\text { academic } \\
\text { procrastinatio } \\
\text { n. }\end{array}$ & \\
\hline $\begin{array}{l}\text { Constantin K. } \\
(2018) \text {. } \\
\text { Anxiety, } \\
\text { depression, } \\
\text { and } \\
\text { procrastinatio } \\
\text { n among } \\
\text { students: } \\
\text { rumination } \\
\text { plays a larger } \\
\text { mediating } \\
\text { role than } \\
\text { worry }\end{array}$ & Canada & $\begin{array}{l}\text { It is a cross- } \\
\text { sectional } \\
\text { study. } \\
\text { Participants: } \\
250 \text { students } \\
\text { who } \\
\text { completed the } \\
\text { Tuckman } \\
\text { Procrastinatio } \\
\text { n Scale (TPS) } \\
\text { and } \\
\text { Difficulties in } \\
\text { Emotion } \\
\text { Regulation } \\
\text { Scale (DERS) }\end{array}$ & $\begin{array}{l}\text { Correlation } \\
\text { analyses }\end{array}$ & $\begin{array}{l}\text { Difficulty in } \\
\text { emotion } \\
\text { regulation is } \\
\text { important in } \\
\text { procrastinatio } \\
\text { n. Despite the } \\
\text { limited } \\
\text { association } \\
\text { between } \\
\text { DERS and } \\
\text { TPS, the } \\
\text { findings raise } \\
\text { some } \\
\text { potentially } \\
\text { useful } \\
\text { implications } \\
\text { for } \\
\text { procrastinatio } \\
\text { n studies and } \\
\text { interventions }\end{array}$ & $4 / * * *$ \\
\hline $\begin{array}{l}\text { Eckert } \\
\text { M.(2016). } \\
\text { Overcome } \\
\text { procrastinatio } \\
\text { n: enhancing } \\
\text { emotion }\end{array}$ & Germany & $\begin{array}{l}\text { Aimed to test } \\
\text { the } \\
\text { hypothesis } \\
\text { that the } \\
\text { availability of } \\
\text { adaptive }\end{array}$ & $\begin{array}{l}\text { Cross- } \\
\text { sectional } \\
\text { analyses of } \\
\text { first study. } \\
\text { Cross lagged } \\
\text { panel analysis }\end{array}$ & $\begin{array}{l}\text { ER skills and } \\
\text { procrastinatio } \\
\mathrm{n} \text { were } \\
\text { associated } \\
\text { and that these } \\
\text { connections }\end{array}$ & $2 / * * * * * *$ \\
\hline
\end{tabular}




\begin{tabular}{|c|c|c|c|c|c|}
\hline $\begin{array}{l}\text { regulation } \\
\text { skills reduce } \\
\text { procrastinatio } \\
\text { n }\end{array}$ & & $\begin{array}{l}\text { emotion } \\
\text { regulation } \\
\text { (ER) skills } \\
\text { prevents } \\
\text { procrastinatio } \\
\text { n. }\end{array}$ & $\begin{array}{l}\text { of second } \\
\text { study. Two- } \\
\text { arm } \\
\text { randomized } \\
\text { control trial } \\
\text { (RCT) was } \\
\text { conducted in } \\
\text { the third } \\
\text { study }\end{array}$ & $\begin{array}{l}\text { were } \\
\text { mediated by } \\
\text { the ability to } \\
\text { tolerate } \\
\text { aversive } \\
\text { emotions. } \\
\text { The ability to } \\
\text { modify } \\
\text { aversive } \\
\text { emotions } \\
\text { reduced } \\
\text { subsequent } \\
\text { procrastinatio } \\
\text { n and } \\
\text { procrastinatio } \\
\text { n affected the } \\
\text { subsequent } \\
\text { ability to } \\
\text { tolerate } \\
\text { aversive } \\
\text { emotion. } \\
\text { Systematic } \\
\text { training of the } \\
\text { ER skills to } \\
\text { tolerate and } \\
\text { modify } \\
\text { aversive } \\
\text { emotions } \\
\text { reduced } \\
\text { procrastinatio } \\
\text { n. Thus, in } \\
\text { order to } \\
\text { overcome } \\
\text { procrastinatio } \\
\text { n, emotion- } \\
\text { focused } \\
\text { strategies } \\
\text { should be } \\
\text { considered }\end{array}$ & \\
\hline $\begin{array}{l}\text { Ozer B.U. } \\
\text { Exploring } \\
\text { academic } \\
\text { procrastinatio } \\
\text { n among } \\
\text { Turkish }\end{array}$ & Turkey & $\begin{array}{l}\text { Examined the } \\
\text { prevalence of } \\
\text { and reasons } \\
\text { for academic } \\
\text { procrastinatio } \\
\mathrm{n} \text { as a }\end{array}$ & $\begin{array}{l}\text { Turkish } \\
\text { Procrastinatio } \\
\text { n Assessment } \\
\text { Scale- } \\
\text { Students }\end{array}$ & $\begin{array}{l}52 \% \text { of } \\
\text { students self- } \\
\text { reported } \\
\text { frequent } \\
\text { academic } \\
\text { procrastinatio }\end{array}$ & $4 / * * * * * *$ \\
\hline
\end{tabular}




\begin{tabular}{|c|c|c|c|c|c|}
\hline $\begin{array}{l}\text { students: } \\
\text { Possible } \\
\text { gender } \\
\text { differences in } \\
\text { prevalence } \\
\text { and reasons. }\end{array}$ & & $\begin{array}{l}\text { function of } \\
\text { gender and } \\
\text { academic } \\
\text { grade level. } \\
\text { Participants: } \\
\text { Study } 1 \text { - } 203 \\
\text { Turkish } \\
\text { undergraduat } \\
\text { e students. } \\
\text { Study } 2 \text { - } 784 \\
\text { students (363 } \\
\text { women and } \\
421 \text { men) }\end{array}$ & & $\begin{array}{l}\text { n. Males } \\
\text { reported more } \\
\text { frequent } \\
\text { procrastinatio } \\
\text { n on } \\
\text { academic } \\
\text { tasks than } \\
\text { females. } \\
\text { Significantly } \\
\text { more females } \\
\text { than males } \\
\text { reported } \\
\text { greater } \\
\text { academic } \\
\text { procrastinatio } \\
\text { n due to fear } \\
\text { of failure and } \\
\text { laziness. } \\
\text { Males } \\
\text { reported more } \\
\text { academic } \\
\text { procrastinatio } \\
\text { n as a result } \\
\text { of risk tasing } \\
\text { and rebellion } \\
\text { against } \\
\text { control than } \\
\text { did females }\end{array}$ & \\
\hline $\begin{array}{l}\text { Ferrari J. R. } \\
(1995) \text {. } \\
\text { Methods of } \\
\text { procrastinatio } \\
\mathrm{n} \text { and their } \\
\text { relation to } \\
\text { self-control } \\
\text { and self- } \\
\text { reinforcement } \\
\text { : an } \\
\text { exploratory } \\
\text { study. }\end{array}$ & United States & $\begin{array}{l}\text { Participants } \\
\text { consisted of } \\
277 \text { college } \\
\text { students ( } 205 \\
\text { female and } 72 \\
\text { male) who } \\
\text { were scored on } \\
\text { procrastination } \\
\text { measures } \\
\text { through a } \\
\text { series of } \\
\text { questionnaires }\end{array}$ & $\begin{array}{l}\text { The Decisional } \\
\text { Procrastination } \\
\text { Scale, The } \\
\text { Adult } \\
\text { Inventory of } \\
\text { Procrastination } \\
\text { The Self- } \\
\text { Control Scale, } \\
\text { Frequency of } \\
\text { Self- } \\
\text { Reinforcement } \\
\text { Scale }\end{array}$ & $\begin{array}{l}\text { Despite } \\
\text { different } \\
\text { methods of } \\
\text { procrastination } \\
\text {, all forms } \\
\text { seem to } \\
\text { correlate with } \\
\text { the need of } \\
\text { immediate } \\
\text { pleasure and } \\
\text { the avoidance } \\
\text { of pain. }\end{array}$ & $4 / * * * * *$ \\
\hline $\begin{array}{l}\text { Glick D.M. } \\
\text { (2013) A } \\
\text { preliminary } \\
\text { investigation }\end{array}$ & United States & $\begin{array}{l}\text { Aimed to } \\
\text { investigate } \\
\text { the } \\
\text { associations }\end{array}$ & $\begin{array}{l}\text { Procrastinatio } \\
\text { n Assessment } \\
\text { Scale- } \\
\text { Students, }\end{array}$ & $\begin{array}{l}\text { There are } \\
\text { significant } \\
\text { associations } \\
\text { among school }\end{array}$ & $4 / * * * * *$ \\
\hline
\end{tabular}




\begin{tabular}{|c|c|c|c|c|c|}
\hline $\begin{array}{l}\text { of the role of } \\
\text { psychological } \\
\text { inflexibility } \\
\text { in academic } \\
\text { procrastinatio } \\
n\end{array}$ & & $\begin{array}{l}\text { between } \\
\text { school } \\
\text { attachment, } \\
\text { academic } \\
\text { procrastinatio } \\
\text { n and life } \\
\text { satisfaction } \\
\text { and the } \\
\text { mediating } \\
\text { effect of } \\
\text { academic } \\
\text { procrastinatio } \\
\text { n. } \\
\text { Participants: } \\
\text { The } \\
\text { mediation } \\
\text { model was } \\
\text { tested with } \\
324 \\
\text { adolescents } \\
\text { (53.4\% } \\
\text { female, and } \\
46.6 \% \text { male) }\end{array}$ & $\begin{array}{l}\text { State-trait } \\
\text { Anxiety } \\
\text { Inventory- } \\
\text { Trait, Action } \\
\text { and } \\
\text { acceptance } \\
\text { Questionnaire } \\
\text {, Academic } \\
\text { Values } \\
\text { Questionnaire }\end{array}$ & $\begin{array}{l}\text { attachment, } \\
\text { academiC } \\
\text { procrastinatio } \\
\mathrm{n} \text { and life } \\
\text { satisfaction. } \\
\text { The results } \\
\text { show that } \\
\text { school } \\
\text { attachment } \\
\text { contributes to } \\
\text { a reduction in } \\
\text { academic } \\
\text { procrastinatio } \\
\text { n and an } \\
\text { increase in } \\
\text { life } \\
\text { satisfaction. } \\
\text { In this } \\
\text { respect, it can } \\
\text { be said that } \\
\text { attachment to } \\
\text { school has a } \\
\text { therapeutic } \\
\text { value for } \\
\text { students }\end{array}$ & \\
\hline $\begin{array}{l}\text { Häfner A. } \\
\text { Avoiding } \\
\text { procrastinatio } \\
\text { n through time } \\
\text { management: } \\
\text { an } \\
\text { experimental } \\
\text { intervention } \\
\text { study. }\end{array}$ & Germany & $\begin{array}{l}\text { Examined the } \\
\text { effects of } \\
\text { short term } \\
\text { time } \\
\text { management } \\
\text { intervention } \\
\text { on } \\
\text { procrastinatio } \\
\text { n. Randomly } \\
\text { assigned } 96 \\
\text { students to a } \\
\text { treatment and } \\
\text { control group. }\end{array}$ & & $\begin{array}{l}\text { Participants } \\
\text { of the control } \\
\text { group showed } \\
\text { procrastinatio } \\
\mathrm{n} \text {, and } \\
\text { participants } \\
\text { of the } \\
\text { treatment } \\
\text { group } \\
\text { allocated } \\
\text { their work } \\
\text { time more } \\
\text { equally and } \\
\text { did not show } \\
\text { procrastinatio } \\
\text { n. Therefore, } \\
\text { the explored } \\
\text { time } \\
\text { management } \\
\text { intervention }\end{array}$ & $2 / * *$ \\
\hline
\end{tabular}




\begin{tabular}{|c|c|c|c|c|c|}
\hline & & & & $\begin{array}{l}\text { prevents from } \\
\text { a serious time } \\
\text { management } \\
\text { problem }\end{array}$ & \\
\hline $\begin{array}{l}\text { Haghbin, M. } \\
\text { The } \\
\text { Complexity } \\
\text { of the } \\
\text { Relation } \\
\text { between Fear } \\
\text { of Failure and } \\
\text { Procrastinatio } \\
n\end{array}$ & Canada & $\begin{array}{l}\text { Investigated } \\
\text { the indirect } \\
\text { and } \\
\text { conditional } \\
\text { relation } \\
\text { between fear } \\
\text { of failure and } \\
\text { procrastinatio } \\
\text { n based on } \\
\text { constructs } \\
\text { from self- } \\
\text { determination } \\
\text { theory. } \\
\text { Participants: } \\
300 \\
\text { university } \\
\text { students }\end{array}$ & $\begin{array}{l}\text { Used } \\
\text { structural } \\
\text { equation } \\
\text { modeling to } \\
\text { analyze data }\end{array}$ & $\begin{array}{l}\text { The relation } \\
\text { between fear } \\
\text { of failure and } \\
\text { procrastinatio } \\
\text { n was } \\
\text { moderated by } \\
\text { perceived } \\
\text { competence. } \\
\text { The relation } \\
\text { was } \\
\text { positively } \\
\text { significant for } \\
\text { students with } \\
\text { low levels of } \\
\text { competence } \\
\text { and } \\
\text { negatively } \\
\text { significant for } \\
\text { those with } \\
\text { high level of } \\
\text { competence }\end{array}$ & $4 / * * *$ \\
\hline $\begin{array}{l}\text { Chun Chu, } \\
\text { A.H. 2005. } \\
\text { Rethinking } \\
\text { Procrastinatio } \\
\text { n: Positive } \\
\text { Effects of } \\
\text { "Active" } \\
\text { Procrastinatio } \\
\text { n Behavior on } \\
\text { Attitudes and } \\
\text { Performance. }\end{array}$ & Canada & $\begin{array}{l}\mathrm{n}=230 \text { (166 } \\
\text { women, } 64 \\
\text { men) from } 3 \\
\text { Canadian } \\
\text { universities } \\
\text { filled out a } \\
\text { series of } \\
\text { questionnaire } \\
\text { s. The } \\
\text { purpose of } \\
\text { the study was } \\
\text { to identify a } \\
\text { positive type } \\
\text { of } \\
\text { procrastinator } \\
\text { (active } \\
\text { procrastinator } \\
\text { s) }\end{array}$ & $\begin{array}{l}\text { Quantitative } \\
\text { descriptive } \\
\text { study: } \\
\text { questionnaire } \\
\text { s using a 7- } \\
\text { point Likert- } \\
\text { type scale. } \\
\text { They adopted } \\
\text { items from } \\
\text { different } \\
\text { scales to } \\
\text { measure each } \\
\text { parameter: } \\
\text { academic } \\
\text { procrastinatio } \\
n \text {, active } \\
\text { procrastinatio } \\
n, \text { patterns of } \\
\text { time use, }\end{array}$ & $\begin{array}{l}\text { The authors } \\
\text { identified two } \\
\text { distinct } \\
\text { groups of } \\
\text { procrastinator } \\
\text { s: passive vs } \\
\text { active } \\
\text { procrastinator } \\
\text { s. Active } \\
\text { procrastinator } \\
\text { s share a } \\
\text { similar level } \\
\text { of } \\
\text { procrastinatio } \\
\text { n with } \\
\text { passive } \\
\text { procrastinatio } \\
\text { n however } \\
\text { they more }\end{array}$ & $4 / * * * * * *$ \\
\hline
\end{tabular}




\begin{tabular}{|c|c|c|c|c|c|}
\hline & & & $\begin{array}{l}\text { perception of } \\
\text { time control, } \\
\text { self efficacy } \\
\text { belief, } \\
\text { motivational } \\
\text { orientations, } \\
\text { stress-coping } \\
\text { strategy, } \\
\text { stress, } \\
\text { depression, } \\
\text { life } \\
\text { satisfaction, } \\
\text { and academic } \\
\text { performance }\end{array}$ & $\begin{array}{l}\text { closely } \\
\text { resemble } \\
\text { non- } \\
\text { procrastinator } \\
\text { s in terms of } \\
\text { use of time, } \\
\text { self efficacy } \\
\text { belief, coping } \\
\text { styles, } \\
\text { academic } \\
\text { performance } \\
\text { and } \\
\text { outcomes. }\end{array}$ & \\
\hline $\begin{array}{l}\text { Zacks, S., } \\
2018 . \\
\text { Academic } \\
\text { interventions } \\
\text { for academic } \\
\text { procrastinatio } \\
\text { n: A review } \\
\text { of the } \\
\text { literature. }\end{array}$ & Israel & $\begin{array}{l}\text { Literature } \\
\text { review to } \\
\text { determine the } \\
\text { causes and } \\
\text { consequences } \\
\text { of } \\
\text { procrastinatio } \\
\mathrm{n} \text { as well as } \\
\text { effective } \\
\text { procrastinatio } \\
\mathrm{n} \\
\text { interventions }\end{array}$ & $\begin{array}{l}\text { Literature } \\
\text { review }\end{array}$ & $\begin{array}{l}\text { The authors } \\
\text { concluded } \\
\text { that more } \\
\text { research on } \\
\text { effective } \\
\text { teaching } \\
\text { strategies and } \\
\text { procrastinatio } \\
\text { n intervention } \\
\text { is necessary. } \\
\text { Instructional } \\
\text { intervention } \\
\text { tends to be } \\
\text { effective as it } \\
\text { helps with the } \\
\text { failure to self- } \\
\text { regulate in } \\
\text { procrastinator } \\
\text { s. }\end{array}$ & $1 / * * * *$ \\
\hline $\begin{array}{l}\text { Svartdal F, } \\
(2020) \text {. How } \\
\text { Study } \\
\text { Environments } \\
\text { Foster } \\
\text { Academic } \\
\text { Procrastinatio } \\
\text { n: Overview } \\
\text { and } \\
\text { Recommenda } \\
\text { tions. }\end{array}$ & Germany & $\begin{array}{l}\text { Created and } \\
\text { evaluated a } \\
\text { group } \\
\text { training based } \\
\text { on a cyclical } \\
\text { process } \\
\text { model of self- } \\
\text { regulated } \\
\text { learning to } \\
\text { reduce } \\
\text { procrastinatio }\end{array}$ & $\begin{array}{l}\text { Mixed } \\
\text { Methods: } \\
\text { Participants } \\
\text { engaged in } \\
\text { weekly } \\
\text { sessions each } \\
\text { with a } \\
\text { specific goal } \\
\text { (introduction, } \\
\text { goal and time } \\
\text { management, }\end{array}$ & $\begin{array}{l}\text { Students gave } \\
\text { the group } \\
\text { training a } \\
\text { good } \\
\text { evaluations } \\
\text { and found it } \\
\text { would be } \\
\text { helpful for } \\
\text { peers } \\
\text { suffering with } \\
\text { procrastinatio }\end{array}$ & $5 / * * * *$ \\
\hline
\end{tabular}




\begin{tabular}{|c|c|c|c|c|c|}
\hline & & $\begin{array}{l}\mathrm{n} \text { in students } \\
(\mathrm{n}=106)\end{array}$ & $\begin{array}{l}\text { self- } \\
\text { motivation, } \\
\text { distractions } \\
\text { and } \\
\text { temptations, } \\
\text { and } \\
\text { preparation } \\
\text { for the } \\
\text { future). } \\
\text { Students } \\
\text { completed a } \\
\text { modified 16- } \\
\text { item } \\
\text { Tuckman } \\
\text { procrastinatio } \\
\text { n scale, } \\
\text { subscales of } \\
\text { the German } \\
\text { adaptation of } \\
\text { the motivated } \\
\text { strategies for } \\
\text { learning } \\
\text { questionnaire, } \\
\text { and a } \\
\text { questionnaire } \\
\text { to evaluate } \\
\text { the training. }\end{array}$ & $\mathrm{n}$ & \\
\hline $\begin{array}{l}\text { Jackson, T. } \\
(2003) \text {. } \\
\text { Procrastinatio } \\
\text { n and } \\
\text { perceptions } \\
\text { of past, } \\
\text { present, and } \\
\text { future. }\end{array}$ & N/A & $\begin{array}{l}\text { Evaluated } \\
\text { assumptions } \\
\text { of self- } \\
\text { regulation } \\
\text { and specious } \\
\text { rewards } \\
\text { explanations } \\
\text { of } \\
\text { procrastinatio } \\
\text { n which } \\
\text { postulate that } \\
\text { a reduced } \\
\text { focus on the } \\
\text { future among } \\
\text { procrastinator } \\
\text { s is due to } \\
\text { their } \\
\text { increased }\end{array}$ & $\begin{array}{l}\text { Quantitative } \\
\text { descriptive } \\
\text { study: } \\
\text { questionnaire } \\
\text { s }\end{array}$ & $\begin{array}{l}\text { Explanations } \\
\text { invoking } \\
\text { failures in } \\
\text { self- } \\
\text { regulation or } \\
\text { pre- } \\
\text { occupations } \\
\text { with specious } \\
\text { rewards to } \\
\text { account for } \\
\text { procrastinator } \\
\text { s' reduced } \\
\text { focus on } \\
\text { setting and } \\
\text { carrying out } \\
\text { future goals } \\
\text { require } \\
\text { modification }\end{array}$ & $4 / * * * * * *$ \\
\hline
\end{tabular}




\begin{tabular}{|c|c|c|c|c|c|}
\hline & & $\begin{array}{l}\text { focus on } \\
\text { current } \\
\text { concerns and } \\
\text { immediate } \\
\text { rewards. } \\
\text { Study } 1 \\
\text { (n=1147) } \\
\text { self-report } \\
\text { measures of } \\
\text { procrastinatio } \\
\text { n and past, } \\
\text { present and } \\
\text { future time } \\
\text { perspectives. } \\
\text { Study } 2 \\
\text { (n=160) } \\
\text { controlled for } \\
\text { negative } \\
\text { affect. }\end{array}$ & & & \\
\hline $\begin{array}{l}\text { Kandemir M. } \\
(2014) \\
\text { Reasons of } \\
\text { academic } \\
\text { procrastinatio } \\
\text { n: self- } \\
\text { regulation, } \\
\text { academic } \\
\text { self-efficacy, } \\
\text { life } \\
\text { satisfaction } \\
\text { and } \\
\text { demographics } \\
\text { variables }\end{array}$ & Turkey & $\begin{array}{l}619 \text { from } \\
\text { Kirikkale } \\
\text { University } \\
\text { took part in a } \\
\text { survey, <one } \\
\text { day }\end{array}$ & $\begin{array}{l}\text { Quantitative } \\
\text { descriptive } \\
\text { study: } \\
\text { questionnaire } \\
\text { s }\end{array}$ & $\begin{array}{l}\text { Explanations } \\
\text { invoking } \\
\text { failures in } \\
\text { self- } \\
\text { regulation or } \\
\text { pre- } \\
\text { occupations } \\
\text { with specious } \\
\text { rewards to } \\
\text { account for } \\
\text { procrastinator } \\
\text { s' reduced } \\
\text { focus on } \\
\text { setting and } \\
\text { carrying out } \\
\text { future goals } \\
\text { require } \\
\text { modification }\end{array}$ & $4 / * * * * * *$ \\
\hline $\begin{array}{l}\text { Milgram, N. } \\
(1995) \text {. } \\
\text { Correlates of } \\
\text { academic } \\
\text { procrastinatio } \\
\text { n: discomfort, } \\
\text { task }\end{array}$ & Israel & $\begin{array}{l}\text { Studied the } \\
\text { relationships } \\
\text { among five } \\
\text { aspects of } \\
\text { academic } \\
\text { procrastinatio } \\
\text { n- }\end{array}$ & $\begin{array}{l}\text { Quantitative } \\
\text { descriptive } \\
\text { study: } \\
\text { Procrastinatio } \\
\text { n assessment } \\
\text { scale students }\end{array}$ & $\begin{array}{l}\text { Delay on } \\
\text { academic } \\
\text { tasks was } \\
\text { affected by } \\
\text { task } \\
\text { aversiveness } \\
\text { but not by }\end{array}$ & $4 / * * * *$ \\
\hline
\end{tabular}




\begin{tabular}{|c|c|c|c|c|c|}
\hline $\begin{array}{l}\text { aversiveness, } \\
\text { and task } \\
\text { capability. }\end{array}$ & & $\begin{array}{l}\text { behavioural } \\
\text { delay, } \\
\text { personal } \\
\text { upset about } \\
\text { the delay, } \\
\text { task } \\
\text { aversiveness, } \\
\text { task } \\
\text { capability, } \\
\text { and the desire } \\
\text { to reduce } \\
\text { behavioural } \\
\text { delay in 10-th } \\
\text { grade Israeli } \\
\text { students } \\
\text { (n=195) }\end{array}$ & & $\begin{array}{l}\text { task } \\
\text { capability, } \\
\text { upset was } \\
\text { adversely } \\
\text { affected by } \\
\text { low } \\
\text { perceived- } \\
\text { task } \\
\text { capability and } \\
\text { in turn } \\
\text { increased } \\
\text { interest in } \\
\text { reducing } \\
\text { delaying } \\
\text { behaviour, } \\
\text { and the kind } \\
\text { of reasons } \\
\text { given for } \\
\text { delay had } \\
\text { more to do } \\
\text { with social } \\
\text { desirability } \\
\text { than with } \\
\text { delay and } \\
\text { upset patterns }\end{array}$ & \\
\hline $\begin{array}{l}\text { Özer B. U. } \\
2005 \\
\text { Academic } \\
\text { procrastinatio } \\
\text { n: Prevalence, } \\
\text { self-reported } \\
\text { reasons, } \\
\text { gender } \\
\text { difference } \\
\text { and it's } \\
\text { relation with } \\
\text { academic } \\
\text { achievement } \\
\text { (Unpublished } \\
\text { doctorate } \\
\text { dissertation } \\
\text { Unpublished } \\
\text { master } \\
\text { thesis). } \\
\text { Middle East }\end{array}$ & Turkey & $\begin{array}{l}\text { Purpose of } \\
\text { the study is to } \\
\text { examine level } \\
\text { of academic } \\
\text { procrastinatio } \\
\mathrm{n} \text {, prevalence } \\
\text { of } \\
\text { procrastinatio } \\
\mathrm{n} \text { in six } \\
\text { aspects of } \\
\text { academic } \\
\text { work, the } \\
\text { relationship } \\
\text { between } \\
\text { academic } \\
\text { procrastinatio } \\
\text { n score and } \\
\text { academic } \\
\text { achievement, } \\
\text { and }\end{array}$ & $\begin{array}{l}\text { Quantitative } \\
\text { descriptive } \\
\text { study: survey } \\
\text { and } \\
\text { correlational } \\
\text { study. Used } \\
\text { Procrastinatio } \\
\text { n Assessment } \\
\text { Scale- } \\
\text { Students } \\
\text { (PASS), } \\
\text { Aitken } \\
\text { Procrastinatio } \\
\text { n Inventory } \\
\text { (API), } \\
\text { Students } \\
\text { Opinion } \\
\text { Questionnaire }\end{array}$ & $\begin{array}{l}52 \% \text { of } \\
\text { METU } \\
\text { students } \\
\text { procrastinate } \\
\text { on their } \\
\text { academic } \\
\text { tasks and the } \\
\text { findings } \\
\text { revealed that } \\
\text { male students } \\
\text { procrastinate } \\
\text { more than } \\
\text { female } \\
\text { students. } \\
\text { Students } \\
\text { always or } \\
\text { nearly always } \\
\text { engage in } \\
\text { procrastinatio } \\
\text { n in the area }\end{array}$ & $4 / * * * * * *$ \\
\hline
\end{tabular}




\begin{tabular}{|c|c|c|c|c|c|}
\hline $\begin{array}{l}\text { Technical } \\
\text { University } \\
\text { Ankara, } \\
\text { Ankara }\end{array}$ & & $\begin{array}{l}\text { reasonings } \\
\text { behind } \\
\text { procrastinatio } \\
\mathrm{n} \text { all in } \\
\text { relation to } \\
\text { gender in } \\
\text { undergraduat } \\
\text { e students } \\
(\mathrm{n}=784)\end{array}$ & & $\begin{array}{l}\text { of studying } \\
\text { for an exam, } \\
\text { writing a term } \\
\text { paper, and } \\
\text { reading } \\
\text { weekly } \\
\text { reading } \\
\text { assignments } \\
\text { more than the } \\
\text { other three } \\
\text { areas: } \\
\text { academic } \\
\text { administrativ } \\
\text { e tasks, } \\
\text { attendance } \\
\text { tasks and the } \\
\text { tasks related } \\
\text { to school } \\
\text { activities. } \\
\text { There is a } \\
\text { significant } \\
\text { negative } \\
\text { relationship } \\
\text { between } \\
\text { academic } \\
\text { procrastinatio } \\
\text { n and } \\
\text { academic } \\
\text { achievement. }\end{array}$ & \\
\hline $\begin{array}{l}\text { Rozental A. } \\
(2014) \text {. } \\
\text { Understandin } \\
\text { g and treating } \\
\text { procrastinatio } \\
\text { n: a review of } \\
\text { a common } \\
\text { self- } \\
\text { regulatory } \\
\text { failure. }\end{array}$ & Sweden & $\begin{array}{l}\text { Literature } \\
\text { review }\end{array}$ & $\begin{array}{l}\text { Literature } \\
\text { review } \\
\text { explores the } \\
\text { evidence on } \\
\text { using } \\
\text { cognitive } \\
\text { behaviour } \\
\text { therapy for } \\
\text { procrastinatio } \\
\text { n, discussing } \\
\text { scarcity of } \\
\text { randomized } \\
\text { controlled } \\
\text { trials and lack } \\
\text { of validated } \\
\text { outcome }\end{array}$ & $\begin{array}{l}\text { Different } \\
\text { perspectives } \\
\text { from } \\
\text { motivational } \\
\text { theory, } \\
\text { learning } \\
\text { theory, self- } \\
\text { efficacy } \\
\text { theory, and } \\
\text { biases could } \\
\text { help in } \\
\text { designing } \\
\text { interventions } \\
\text { for } \\
\text { procrastinatio } \\
\mathrm{n}\end{array}$ & $5 / * *$ \\
\hline
\end{tabular}




\begin{tabular}{|c|c|c|c|c|c|}
\hline & & & measures & & \\
\hline $\begin{array}{l}\text { Webb T., } \\
\text { (2010). Using } \\
\text { the internet to } \\
\text { promote } \\
\text { health } \\
\text { behavior } \\
\text { change: a } \\
\text { systematic } \\
\text { review and } \\
\text { meta-analysis } \\
\text { of the impact } \\
\text { of theoretical } \\
\text { basis, use of } \\
\text { behavior } \\
\text { change } \\
\text { techniques, } \\
\text { and mode of } \\
\text { delivery on } \\
\text { efficacy. }\end{array}$ & UK & $\begin{array}{l}\text { Systematic } \\
\text { review: } \\
\text { computerized } \\
\text { search of } \\
\text { databases of } \\
\text { literature } \\
\text { between } 2000 \\
\text { and } 2008\end{array}$ & $\begin{array}{l}\text { Studies that } \\
\text { explored } \\
\text { interventions } \\
\text { via the } \\
\text { Internet with } \\
\text { random } \\
\text { assignment of } \\
\text { conditions } \\
\text { and } \\
\text { measurement } \\
\text { of health- } \\
\text { related } \\
\text { behaviors }\end{array}$ & $\begin{array}{l}85 \text { studies } \\
\text { satisfied the } \\
\text { inclusion } \\
\text { criteria; total } \\
\text { sample size } \\
43,236 \text {. } \\
\text { Interventions } \\
\text { had a } \\
\text { statistically } \\
\text { significant } \\
\text { effect on } \\
\text { health-related } \\
\text { behaviour. }\end{array}$ & $4 / * * * * * *$ \\
\hline
\end{tabular}

*Chart continued below

\begin{tabular}{|c|c|c|c|c|c|}
\hline $\begin{array}{l}\text { Wohl M. J. } \\
\text { A. (2010). I } \\
\text { forgive } \\
\text { myself, now I } \\
\text { can study: } \\
\text { how self- } \\
\text { forgiveness } \\
\text { for } \\
\text { procrastinatin } \\
\text { g can reduce } \\
\text { future } \\
\text { procrastinatio } \\
\text { n. }\end{array}$ & & $\begin{array}{l}\text { University } \\
\text { students } \\
(\mathrm{n}=119) \\
\text { completed } \\
\text { questionnaire } \\
\text { s before each } \\
\text { of their } \\
\text { midterms } \\
\text { (total 2) in } \\
\text { their } \\
\text { introductory } \\
\text { psychology } \\
\text { course }\end{array}$ & $\begin{array}{l}\text { Quantitative } \\
\text { descriptive } \\
\text { study: } \\
\text { questionnaire } \\
\text { s measuring } \\
\text { procrastinatio } \\
\text { n and self- } \\
\text { forgiveness }\end{array}$ & $\begin{array}{l}\text { Students who } \\
\text { reported } \\
\text { higher levels } \\
\text { of self- } \\
\text { forgiveness } \\
\text { for } \\
\text { procrastinatin } \\
\text { g on studying } \\
\text { for their first } \\
\text { midterm } \\
\text { showed } \\
\text { reduced } \\
\text { levels of } \\
\text { procrastinatio } \\
n \text { for their } \\
\text { second } \\
\text { midterm }\end{array}$ & $4 / * * * *$ \\
\hline $\begin{array}{l}\text { Aznar-Díaz, } \\
\text { I.(2020) } \\
\text { Mexican and } \\
\text { Spanish }\end{array}$ & $\begin{array}{l}\text { Mexico and } \\
\text { Spain }\end{array}$ & $\begin{array}{l}\text { Cross } \\
\text { sectional } \\
\text { study design } \\
\text { using an }\end{array}$ & $\begin{array}{l}\text { The } \\
\text { questionnaire } \\
\text { contained } \\
\text { three }\end{array}$ & $\begin{array}{l}\text { There were } \\
\text { similar } \\
\text { prevalence } \\
\text { rates of daily }\end{array}$ & $4 / * * * * * *$ \\
\hline
\end{tabular}




\begin{tabular}{|c|c|c|c|c|c|}
\hline $\begin{array}{l}\text { university } \\
\text { students' } \\
\text { Internet } \\
\text { addiction and } \\
\text { academic } \\
\text { procrastinatio } \\
\text { n: Correlation } \\
\text { and potential } \\
\text { factors. }\end{array}$ & & $\begin{array}{l}\text { online } \\
\text { questionnaire } \\
\text { to explore } \\
\text { academic } \\
\text { procrastinatio } \\
n \text { in } \\
\text { university } \\
\text { students } \\
(\mathrm{n}=758)\end{array}$ & $\begin{array}{l}\text { sections: } \\
\text { sociodemogra } \\
\text { phic data, } \\
\text { Internet } \\
\text { Addiction } \\
\text { Test, and } \\
\text { Academic } \\
\text { Procrastinatio } \\
\text { n Scale }\end{array}$ & $\begin{array}{l}\text { internet use in } \\
\text { both students } \\
\text { from Mexico, } \\
\text { Spain, and in } \\
\text { total.Positive } \\
\text { correlation } \\
\text { between } \\
\text { problematic } \\
\text { internet use } \\
\text { and academic } \\
\text { procrastinatio } \\
\text { n. }\end{array}$ & \\
\hline $\begin{array}{l}\text { Balkis, M. } \\
\text { (2012) } \\
\text { Analysis of } \\
\text { the relation } \\
\text { between } \\
\text { academic } \\
\text { procrastinatio } \\
\text { n, academic } \\
\text { rational/irrati } \\
\text { onal beliefs, } \\
\text { time } \\
\text { preferences to } \\
\text { study for } \\
\text { exams, and } \\
\text { academic } \\
\text { achievement: } \\
\text { a structural } \\
\text { model. }\end{array}$ & Turkey & $\begin{array}{l}\text { Questionnaire } \\
\text { s were filled } \\
\text { out by } \\
\text { undergraduat } \\
\text { e students } \\
\text { (n=281) at a } \\
7 \text {-week long } \\
\text { summer } \\
\text { course }\end{array}$ & $\begin{array}{l}\text { Quantitative } \\
\text { descriptive } \\
\text { study: } \\
\text { questionnaire } \\
\text { s measuring } \\
\text { academic } \\
\text { procrastinatio } \\
\text { n, academic } \\
\text { rational/irrati } \\
\text { onal beliefs, } \\
\text { and time } \\
\text { preferences to } \\
\text { study for } \\
\text { exams }\end{array}$ & $\begin{array}{l}\text { Rational } \\
\text { academic } \\
\text { beliefs have a } \\
\text { direct impact } \\
\text { on academic } \\
\text { procrastinatio } \\
\text { n and time } \\
\text { preferences to } \\
\text { study for } \\
\text { exams. } \\
\text { Academic } \\
\text { procrastinatio } \\
\text { n had a direct } \\
\text { impact on } \\
\text { academic } \\
\text { achievement. }\end{array}$ & $4 / * * * * * *$ \\
\hline $\begin{array}{l}\text { Balkis, M. } \\
\text { Procrastinatio } \\
\mathrm{n} \text { and } \\
\text { Rational/Irrati } \\
\text { onal Beliefs: } \\
\text { A Moderated } \\
\text { Mediation } \\
\text { Model. }\end{array}$ & Turkey & $\begin{array}{l}\text { Undergraduat } \\
\text { e students } \\
\text { ( } n=293 \text { ) filled } \\
\text { out a series of } \\
\text { questionnaire } \\
\text { s and } \\
\text { demographic } \\
\text { information } \\
\text { for } 20 \\
\text { minutes }\end{array}$ & $\begin{array}{l}\text { Quantitative } \\
\text { descriptive } \\
\text { study: } \\
\text { questionnaire } \\
\text { s about } \\
\text { demographic, } \\
\text { self-doubt, } \\
\text { general } \\
\text { attitude and } \\
\text { belief, pure } \\
\text { procrastinatio } \\
\text { n, and } \\
\text { performance } \\
\text { failure }\end{array}$ & $\begin{array}{l}\text { Results show } \\
\text { that self } \\
\text { doubt and } \\
\text { irrational } \\
\text { beliefs have } \\
\text { direct and } \\
\text { interactive } \\
\text { effects on } \\
\text { fear of } \\
\text { failure, fear } \\
\text { of failure } \\
\text { mediates } \\
\text { relationship } \\
\text { between self- }\end{array}$ & $4 / * * * * * *$ \\
\hline
\end{tabular}




\begin{tabular}{|c|c|c|c|c|c|}
\hline & & & $\begin{array}{l}\text { appraisal } \\
\text { inventory }\end{array}$ & $\begin{array}{l}\text { doubt and } \\
\text { procrastinatio } \\
\mathrm{n} \text {, rational } \\
\text { beliefs } \\
\text { moderated } \\
\text { the predictive } \\
\text { effect of fear } \\
\text { of failure on } \\
\text { procrastinatio } \\
\mathrm{n} \text {, and the } \\
\text { indirect effect } \\
\text { of self-doubt } \\
\text { on } \\
\text { procrastinatio } \\
\mathrm{n} \text { via fear } \\
\text { may depend } \\
\text { on the level } \\
\text { of rational } \\
\text { and irrational } \\
\text { beliefs }\end{array}$ & \\
\hline $\begin{array}{l}\text { Bennett, C., } \\
\text { \& Bacon, A. } \\
\text { M. (2019). At } \\
\text { long last-A } \\
\text { reinforcement } \\
\text { sensitivity } \\
\text { theory } \\
\text { explanation } \\
\text { of } \\
\text { procrastinatio } \\
\text { n. Journal of } \\
\text { Individual } \\
\text { Differences. }\end{array}$ & England & $\begin{array}{l}\text { Students } \\
(\mathrm{n}=336) \text { and } \\
\text { non-students } \\
(\mathrm{n}=187) \text { ) } \\
\text { completed a } \\
\text { series of } \\
\text { questionnaire } \\
\text { s to examine } \\
\text { procrastinatio } \\
\text { n through the } \\
\text { lens of } \\
\text { reinforcement } \\
\text { sensitivity } \\
\text { theory }\end{array}$ & $\begin{array}{l}\text { Quantitative } \\
\text { descriptive } \\
\text { study: } \\
\text { questionnaire } \\
\text { s about } \\
\text { personality, } \\
\text { procrastinatio } \\
\text { n, high } \\
\text { impulsivity } \\
\text { and } \\
\text { behavioural } \\
\text { inhibition etc. }\end{array}$ & $\begin{array}{l}\text { high } \\
\text { impulsivity } \\
\text { and high } \\
\text { behavioural } \\
\text { inhibition } \\
\text { system } \\
\text { activation } \\
\text { were } \\
\text { associated } \\
\text { with reports } \\
\text { of general } \\
\text { procrastinatio } \\
\text { n. } \\
\text { Unintentional } \\
\text { reward } \\
\text { reactivity was } \\
\text { associated } \\
\text { with } \\
\text { unintentional } \\
\text { procrastinatio } \\
\text { n in students. }\end{array}$ & $4 / * * * * * *$ \\
\hline $\begin{array}{l}\text { Blouin } \square \text { Hud } \\
\text { on, E. M. C. } \\
\text { (2017). A }\end{array}$ & Canada & $\begin{array}{l}\text { Undergraduat } \\
\text { e students } \\
(n=193) \text { were }\end{array}$ & $\begin{array}{l}\text { They } \\
\text { completed } \\
\text { pre and post- }\end{array}$ & $\begin{array}{l}\text { Perceiving } \\
\text { future self } \\
\text { from a }\end{array}$ & $2 / * * * * * *$ \\
\hline
\end{tabular}




\begin{tabular}{|c|c|c|c|c|c|}
\hline $\begin{array}{l}\text { mental } \\
\text { imagery } \\
\text { intervention } \\
\text { to increase } \\
\text { future } \\
\text { self } \square \text { continui } \\
\text { ty and reduce } \\
\text { procrastinatio } \\
\text { n }\end{array}$ & & $\begin{array}{l}\text { randomly } \\
\text { assigned to a } \\
\text { present- } \\
\text { focused } \\
\text { meditation or } \\
\text { future- } \\
\text { focused } \\
\text { mental } \\
\text { imagery } \\
\text { condition for } \\
\text { four weeks, } \\
\text { twice a week. }\end{array}$ & $\begin{array}{l}\text { intervention } \\
\text { questionnaire } \\
\text { s and a half- } \\
\text { point } \\
\text { questionnaire. }\end{array}$ & $\begin{array}{l}\text { cognitive } \\
\text { perspective } \\
\text { seems to be } \\
\text { the most } \\
\text { effective at } \\
\text { increasing an } \\
\text { altruistic } \\
\text { motivation } \\
\text { towards that } \\
\text { self by } \\
\text { procrastinatin } \\
\text { g less in the } \\
\text { present }\end{array}$ & \\
\hline $\begin{array}{l}\text { Boysan, M. } \\
(2017) \text {. } \\
\text { Associations } \\
\text { between } \\
\text { procrastinatio } \\
\text { n, } \\
\text { personality, } \\
\text { perfectionism } \\
\text {, self-esteem } \\
\text { and locus of } \\
\text { control. }\end{array}$ & UK & $\begin{array}{l}\text { Young adults } \\
(\mathrm{n}=242) \\
\text { completed } \\
\text { questionnaire } \\
\mathrm{s}\end{array}$ & $\begin{array}{l}\text { Quantitative } \\
\text { descriptive } \\
\text { study: } \\
\text { questionnaire } \\
\text { s about } \\
\text { procrastinatio } \\
\text { n, } \\
\text { perfectionism } \\
\text {, big five } \\
\text { personality, } \\
\text { locus of } \\
\text { control, and } \\
\text { self esteem }\end{array}$ & $\begin{array}{l}\text { Personality- } \\
\text { based } \\
\text { intervention } \\
\text { approaches } \\
\text { with the } \\
\text { inclusion of } \\
\text { family can be } \\
\text { more } \\
\text { influential in } \\
\text { preventing } \\
\text { procrastinatio } \\
n,\end{array}$ & $4 / * * * * * *$ \\
\hline $\begin{array}{l}\text { Bytamar, J. } \\
\text { M.(2020). } \\
\text { Emotion } \\
\text { Regulation } \\
\text { Difficulties } \\
\text { and } \\
\text { Academic } \\
\text { Procrastinatio } \\
\text { n. }\end{array}$ & Iran & $\begin{array}{l}\text { cross } \\
\text { sectional } \\
\text { study design. } \\
\text { Students } \\
\text { (n=250) } \\
\text { completed the } \\
\text { Tuckman } \\
\text { Procrastinatio } \\
\text { n Scale (TPS) } \\
\text { and } \\
\text { Difficulties in } \\
\text { Emotion } \\
\text { Regulation } \\
\text { Scale (DERS) }\end{array}$ & $\begin{array}{l}\text { Quantitative } \\
\text { descriptive } \\
\text { study: } \\
\text { questionnaire } \\
\text { s about } \\
\text { procrastinatio } \\
\text { n and } \\
\text { emotion } \\
\text { regulation, } \\
\text { depression, } \\
\text { and anxiety }\end{array}$ & $\begin{array}{l}\text { Tuckman } \\
\text { Procrastinatio } \\
\mathrm{n} \text { Scale has a } \\
\text { significant } \\
\text { positive } \\
\text { association } \\
\text { on overall } \\
\text { Difficulties in } \\
\text { Emotion } \\
\text { Regulation } \\
\text { Scale }\end{array}$ & $4 / * * * * * *$ \\
\hline $\begin{array}{l}\text { Eckert, M. } \\
\text { (2018). Does } \\
\text { sms-support }\end{array}$ & Germany & $\begin{array}{l}\text { Two week } \\
\text { internet based } \\
\text { training }\end{array}$ & $\begin{array}{l}\text { Randomized } \\
\text { control trial: } \\
\text { three-armed }\end{array}$ & $\begin{array}{l}\text { Short } \\
\text { internet-based } \\
\text { intervention }\end{array}$ & $2 / * * *$ \\
\hline
\end{tabular}




\begin{tabular}{|c|c|c|c|c|c|}
\hline $\begin{array}{l}\text { make a } \\
\text { difference? } \\
\text { effectiveness } \\
\text { of a two- } \\
\text { week online- } \\
\text { training to } \\
\text { overcome } \\
\text { procrastinatio } \\
\text { n. a } \\
\text { randomized } \\
\text { controlled } \\
\text { Trial. }\end{array}$ & & $\begin{array}{l}\text { program } \\
\text { (ON.TOP.) to } \\
\text { overcome } \\
\text { procrastinatio } \\
n \text { in } \\
\text { university } \\
\text { student } \\
(n=161)\end{array}$ & $\begin{array}{l}\text { RCT the } \\
\text { effects of the } \\
\text { intervention } \\
\text { alone and } \\
\text { intervention } \\
\text { with daily } \\
\text { SMS-support } \\
\text { were } \\
\text { compared to a } \\
\text { waiting list } \\
\text { control } \\
\text { condition in a } \\
\text { sample of } \\
\text { students }\end{array}$ & $\begin{array}{l}\text { can help } \\
\text { students } \\
\text { reduce } \\
\text { procrastinatio } \\
\text { n. SMS } \\
\text { support can } \\
\text { increase } \\
\text { efficacy of } \\
\text { interventions }\end{array}$ & \\
\hline $\begin{array}{l}\text { Eisenbeck, } \\
\text { N.(2019). } \\
\text { From } \\
\text { psychological } \\
\text { distress to } \\
\text { academic } \\
\text { procrastinatio } \\
\mathrm{n} \text { : Exploring } \\
\text { the role of } \\
\text { psychological } \\
\text { inflexibility. }\end{array}$ & Spain & $\begin{array}{l}\text { University } \\
\text { students } \\
(\mathrm{n}=429) \\
\text { completed a } \\
\text { series of } \\
\text { questionnaire } \\
\mathrm{s}\end{array}$ & $\begin{array}{l}\text { Quantitative } \\
\text { descriptive } \\
\text { study: } \\
\text { questionnaire } \\
\text { s about } \\
\text { academic } \\
\text { procrastinatio } \\
\text { n, } \\
\text { psychological } \\
\text { inflexibility } \\
\text { and general } \\
\text { psychological } \\
\text { distress } \\
\text { (depression, } \\
\text { anxiety, and } \\
\text { stress) }\end{array}$ & $\begin{array}{l}\text { Higher levels } \\
\text { of } \\
\text { procrastinatio } \\
\text { n were related } \\
\text { to } \\
\text { psychological } \\
\text { distress and } \\
\text { both } \\
\text { procrastinatio } \\
\text { n and } \\
\text { psychological } \\
\text { distress were } \\
\text { related to } \\
\text { psychological } \\
\text { inflexibility }\end{array}$ & $4 / * * * * *$ \\
\hline $\begin{array}{l}\text { Fritzsche, B. } \\
\text { A.(2003). } \\
\text { Individual } \\
\text { differences in } \\
\text { academic } \\
\text { procrastinatio } \\
\text { n tendency } \\
\text { and writing } \\
\text { success. }\end{array}$ & United States & $\begin{array}{l}\text { University } \\
\text { students } \\
(\mathrm{n}=206) \text { in } \\
\text { writing- } \\
\text { intensive } \\
\text { classes } \\
\text { completed a } \\
\text { series of } \\
\text { questionnaire } \\
\mathrm{s}\end{array}$ & $\begin{array}{l}\text { Quantitative } \\
\text { descriptive } \\
\text { study: } \\
\text { questionnaire } \\
\text { s about } \\
\text { procrastinatio } \\
\text { n, anxiety, } \\
\text { writing- } \\
\text { related delay } \\
\text { behaviours } \\
\text { and GPA }\end{array}$ & $\begin{array}{l}\text { The tendency } \\
\text { to } \\
\text { procrastinate } \\
\text { on writing } \\
\text { tasks was } \\
\text { associated } \\
\text { with general } \\
\text { anxiety, anxiet } \\
\text { y about } \\
\text { writing the } \\
\text { paper, less } \\
\text { satisfaction } \\
\text { with writing } \\
\text { the paper, and }\end{array}$ & $4 / * * * * * *$ \\
\hline
\end{tabular}




\begin{tabular}{|c|c|c|c|c|c|}
\hline & & & & lower grades & \\
\hline $\begin{array}{l}\text { Grund, } \\
\text { A.(2018). } \\
\text { Understandin } \\
\text { g } \\
\text { procrastinatio } \\
\text { n: A } \\
\text { motivational } \\
\text { approach. } \\
\text { Personality } \\
\text { and } \\
\text { Individual } \\
\text { Differences, } \\
\text { 121, 120-130. }\end{array}$ & Germany & $\begin{array}{l}\text { Three studies: } \\
\text { Study } 1 \\
\text { (n=223) } \\
\text { completed } \\
\text { questionnaire } \\
\text { s individually } \\
\text {, study } 2 \\
\text { (n=35) } \\
\text { completed } \\
\text { questionnaire } \\
\text { s for seven } \\
\text { days in a } \\
\text { "diary", study } \\
3\end{array}$ & $\begin{array}{l}\text { About self- } \\
\text { determination } \\
\text { and dilatory } \\
\text { about value } \\
\text { orientations } \\
\text { of } \\
\text { procrastinator } \\
\text { s about } \\
\text { ideological } \\
\text { differences in } \\
\text { the attribution } \\
\text { of dilatory } \\
\text { behaviour }\end{array}$ & $\begin{array}{l}\text { Students who } \\
\text { are seen as } \\
\text { problematic } \\
\text { procrastinator } \\
\text { s differ in } \\
\text { their basic } \\
\text { value } \\
\text { orientation } \\
\text { regarding } \\
\text { achievement } \\
\text { and well- } \\
\text { being from } \\
\text { those who } \\
\text { engage less in } \\
\text { dilatory } \\
\text { behaviours. } \\
\text { Levels of self } \\
\text { determination } \\
\text { in everyday } \\
\text { activities are } \\
\text { associated } \\
\text { with } \\
\text { momentary } \\
\text { dilatory } \\
\text { behaviour } \\
\text { and trait } \\
\text { procrastinatio } \\
\text { n. Strong } \\
\text { general } \\
\text { tendency to } \\
\text { attribute } \\
\text { academic } \\
\text { procrastinatio } \\
\text { n to } \\
\text { dispositional } \\
\text { cause. }\end{array}$ & $4 / * * * *$ \\
\hline $\begin{array}{l}\text { Grunschel, } \\
\text { C.(2016) } \\
\text { Exploring } \\
\text { reasons and } \\
\text { consequences } \\
\text { of academic } \\
\text { procrastinatio }\end{array}$ & Germany & $\begin{array}{l}\text { Standardized } \\
\text { interviews } \\
\text { with } \\
\text { university } \\
\text { students } \\
\text { (n=36) who } \\
\text { either }\end{array}$ & $\begin{array}{l}\text { Qualitative } \\
\text { data: } \\
\text { qualitative } \\
\text { content } \\
\text { analysis and } \\
\text { frequency } \\
\text { analysis }\end{array}$ & $\begin{array}{l}\text { The } \\
\text { counselling } \\
\text { group } \\
\text { reported more } \\
\text { serious } \\
\text { reasons and } \\
\text { consequences }\end{array}$ & $5 / * * * * * *$ \\
\hline
\end{tabular}




\begin{tabular}{|c|c|c|c|c|c|}
\hline $\begin{array}{l}\mathrm{n}: \text { an } \\
\text { interview } \\
\text { study. }\end{array}$ & & $\begin{array}{l}\text { belonged or } \\
\text { did not } \\
\text { belong to a } \\
\text { counselling } \\
\text { group }\end{array}$ & & $\begin{array}{l}\text { of academic } \\
\text { procrastinatio } \\
\mathrm{n} \text { than the } \\
\text { non- } \\
\text { counselling } \\
\text { group. }\end{array}$ & \\
\hline $\begin{array}{l}\text { Grunschel, C } \\
\text { (2013). } \\
\text { Exploring } \\
\text { different } \\
\text { types of } \\
\text { academic } \\
\text { delayers: A } \\
\text { latent profile } \\
\text { analysis. } \\
\text { Learning and } \\
\text { Individual } \\
\text { Differences, } \\
\text { 23, 225-233. }\end{array}$ & Germany & $\begin{array}{l}\text { University } \\
\text { students } \\
(\mathrm{n}=554) \\
\text { completed } \\
\text { questionnaire } \\
\text { s and } \\
\text { interviews }\end{array}$ & $\begin{array}{l}\text { Mixed } \\
\text { methods; } \\
\text { Interviews } \\
\text { were used to } \\
\text { assess the } \\
\text { validity of the } \\
\text { questionnaire } \\
\text { s. } \\
\text { Questionnaire } \\
\text { s on academic } \\
\text { procrastinatio } \\
\text { n, big five } \\
\text { personality } \\
\text { traits, } \\
\text { academic } \\
\text { performance, } \\
\text { motivation to } \\
\text { change, and } \\
\text { psychological } \\
\text { strain }\end{array}$ & $\begin{array}{l}\text { They } \\
\text { revealed four } \\
\text { distinct types } \\
\text { of academic } \\
\text { delayers with } \\
\text { a thorough } \\
\text { characterizati } \\
\text { on of each } \\
\text { type. }\end{array}$ & $5 / * * * *$ \\
\hline $\begin{array}{l}\text { Grunschel, C. } \\
\text { (2018). "I'll } \\
\text { stop } \\
\text { procrastinatin } \\
\text { g now!" } \\
\text { Fostering } \\
\text { specific } \\
\text { processes of } \\
\text { self-regulated } \\
\text { learning to } \\
\text { reduce } \\
\text { academic } \\
\text { procrastinatio } \\
\text { n. }\end{array}$ & Germany & $\begin{array}{l}\text { Developed } \\
\text { and evaluated } \\
\text { a group } \\
\text { training to } \\
\text { reduce } \\
\text { academic } \\
\text { procrastinatio } \\
\mathrm{n} \text { in students } \\
\text { ( } \mathrm{n}=106 \text { ). The } \\
\text { training was } \\
\text { made of five } \\
\text { sessions, one } \\
90 \text {-min } \\
\text { session per } \\
\text { week in } \\
\text { groups of } 10 \\
\text { students. }\end{array}$ & $\begin{array}{l}\text { The training } \\
\text { was evaluated } \\
\text { using a } \\
\text { comprehensiv } \\
\text { e control } \\
\text { group design } \\
\text { with repeated } \\
\text { measures }\end{array}$ & $\begin{array}{l}\text { The trained } \\
\text { intervention } \\
\text { group } \\
\text { significantly } \\
\text { reduced } \\
\text { academic } \\
\text { procrastinatio } \\
\text { n and } \\
\text { improved } \\
\text { specific } \\
\text { processes of } \\
\text { self regulated } \\
\text { learning }\end{array}$ & $3 / * * * *$ \\
\hline
\end{tabular}




\begin{tabular}{|c|c|c|c|c|c|}
\hline $\begin{array}{l}\text { Jin, H. } \\
\text { (2019). Peer } \\
\text { attachment } \\
\text { and academic } \\
\text { procrastinatio } \\
\text { n in Chinese } \\
\text { college } \\
\text { students: a } \\
\text { moderated } \\
\text { mediation } \\
\text { model of } \\
\text { future time } \\
\text { perspective } \\
\text { and grit. }\end{array}$ & China & $\begin{array}{l}\text { Chinese } \\
\text { college } \\
\text { students } \\
(\mathrm{n}=1098) \\
\text { filled in a } \\
\text { series of self- } \\
\text { report } \\
\text { questionnaire } \\
\text { s. }\end{array}$ & $\begin{array}{l}\text { Self-report } \\
\text { questionnaire } \\
\text { s about } \\
\text { sociodemogra } \\
\text { phic, } \\
\text { academic } \\
\text { procrastinatio } \\
\text { n, peer } \\
\text { attachment, } \\
\text { grit, future } \\
\text { time } \\
\text { perspective } \\
\text { and analytic } \\
\text { plan }\end{array}$ & $\begin{array}{l}\text { Peer } \\
\text { attachment } \\
\text { was } \\
\text { negatively } \\
\text { associated } \\
\text { with } \\
\text { academic } \\
\text { procrastinatio } \\
\text { n, grit } \\
\text { partially } \\
\text { mediated } \\
\text { association } \\
\text { between peer } \\
\text { attachment } \\
\text { and academic } \\
\text { procrastinatio } \\
\text { n, and future } \\
\text { time } \\
\text { perspective } \\
\text { moderated } \\
\text { the associated } \\
\text { between peer } \\
\text { attachment } \\
\text { and grit }\end{array}$ & $4 / * * * * *$ \\
\hline $\begin{array}{l}\text { Job, } \\
\text { V.(2015). } \\
\text { Implicit } \\
\text { theories about } \\
\text { willpower } \\
\text { predict self- } \\
\text { regulation } \\
\text { and grades in } \\
\text { everyday life. }\end{array}$ & United States & $\begin{array}{l}\text { University } \\
\text { students } \\
\text { ( } \mathrm{n}=101 \text { ) } \\
\text { completed an } \\
\text { online } \\
\text { questionnaire } \\
\text { once a week } \\
\text { during the } \\
\text { second half of } \\
\text { a ten week } \\
\text { term }\end{array}$ & $\begin{array}{l}\text { Quantitative } \\
\text { descriptive } \\
\text { study: } \\
\text { questionnaire } \\
\text { s about } \\
\text { willpower, } \\
\text { self- } \\
\text { regulation } \\
\text { failures and } \\
\text { demands, and } \\
\text { academic } \\
\text { performance } \\
\text { and workload }\end{array}$ & $\begin{array}{l}\text { Better self } \\
\text { regulation } \\
\text { was } \\
\text { associated } \\
\text { with students } \\
\text { who faced } \\
\text { high self } \\
\text { regulatory } \\
\text { demands. } \\
\text { Students } \\
\text { taking a } \\
\text { heavy course } \\
\text { load with a } \\
\text { nonlimite } \\
\text { theory earned } \\
\text { higher grades } \\
\text { due to lower } \\
\text { procrastinatio } \\
\text { n }\end{array}$ & $4 / * * * * * *$ \\
\hline Johnson, J. L. & United States & University & Quantitative & Procrastinatio & $4 / * * * * * *$ \\
\hline
\end{tabular}




\begin{tabular}{|c|c|c|c|c|c|}
\hline $\begin{array}{l}(1995) \text {. An } \\
\text { analysis of } \\
\text { the } \\
\text { contribution } \\
\text { of the five } \\
\text { factors of } \\
\text { personality to } \\
\text { variance in } \\
\text { academic } \\
\text { procrastinatio } \\
\text { n. }\end{array}$ & & $\begin{array}{l}\text { students } \\
(\mathrm{n}=202) \\
\text { completed } \\
\text { questionnaire }\end{array}$ & $\begin{array}{l}\text { descriptive } \\
\text { study: } \\
\text { questionnaire } \\
\text { s - NEO } \\
\text { personality } \\
\text { inventory and } \\
\text { a } \\
\text { procrastinatio } \\
\text { n inventory }\end{array}$ & $\begin{array}{l}\text { n scores were } \\
\text { inversely } \\
\text { related to } \\
\text { conscientious } \\
\text { ness and } \\
\text { significantly } \\
\text { correlated } \\
\text { with } \\
\text { neuroticism }\end{array}$ & \\
\hline $\begin{array}{l}\text { Ko, C. Y. A. } \\
\text { (2019). } \\
\text { Investigating } \\
\text { the } \\
\text { relationships } \\
\text { among } \\
\text { resilience, } \\
\text { social } \\
\text { anxiety, and } \\
\text { procrastinatio } \\
\text { n in a sample } \\
\text { of college } \\
\text { students. }\end{array}$ & Taiwan & $\begin{array}{l}\text { Students } \\
(\mathrm{n}=321) \\
\text { completed } \\
\text { questionnaire } \\
\text { s with two } \\
\text { sections: } \\
\text { demographic } \\
\text { details, and } \\
\text { personal } \\
\text { measures }\end{array}$ & $\begin{array}{l}\text { The second } \\
\text { section of the } \\
\text { questionnaire } \\
\text { asked about } \\
\text { resilience, } \\
\text { social } \\
\text { anxiety, } \\
\text { procrastinatio } \\
\text { n using } \\
\text { different } \\
\text { scales }\end{array}$ & $\begin{array}{l}\text { Students with } \\
\text { higher levels } \\
\text { of resilience } \\
\text { reported } \\
\text { lower } \\
\text { frequency of } \\
\text { procrastinatio } \\
\text { n behaviour. } \\
\text { Resiliency } \\
\text { had an } \\
\text { indirect effect } \\
\text { on } \\
\text { procrastinatio } \\
\text { n through } \\
\text { social anxiety }\end{array}$ & $4 / * * * *$ \\
\hline $\begin{array}{l}\text { Krispenz, A. } \\
\text { (2019). How } \\
\text { to reduce test } \\
\text { anxiety and } \\
\text { academic } \\
\text { procrastinatio } \\
\text { n through } \\
\text { inquiry of } \\
\text { cognitive } \\
\text { appraisals: A } \\
\text { pilot study } \\
\text { investigating } \\
\text { the role of } \\
\text { academic } \\
\text { self-efficacy. }\end{array}$ & Germany & $\begin{array}{l}\text { Longitudinal } \\
\text { quasi- } \\
\text { randomized } \\
\text { intervention } \\
\text { control trial } \\
\text { on } \\
\text { participants } \\
\text { (n=40) who } \\
\text { explored their } \\
\text { worry } \\
\text { thoughts } \\
\text { through IBSR } \\
\text { methods. } \\
\text { Attended a 3- } \\
\text { h IBSR } \\
\text { seminar and } \\
\text { were then } \\
\text { given a diary }\end{array}$ & $\begin{array}{l}\text { Measures } \\
\text { were done } \\
\text { after the } 7- \\
\text { day period } \\
\text { and } \\
\text { immediately } \\
\text { before exams. } \\
\text { Series of } \\
\text { questionnaire } \\
\text { s about } \\
\text { academic self } \\
\text { efficacy, test } \\
\text { anxiety, and } \\
\text { academic } \\
\text { procrastinatio } \\
\text { n }\end{array}$ & $\begin{array}{l}\text { IBSR } \\
\text { intervention } \\
\text { reduced test } \\
\text { anxiety and } \\
\text { academic } \\
\text { procrastinatio } \\
\text { n }\end{array}$ & $2 / * * *$ \\
\hline
\end{tabular}




\begin{tabular}{|l|l|l|l|l|}
\hline & to explore & & \\
worry & & & \\
thoughts via & & & \\
IBSR & & & \\
methods for & & & & \\
\hline
\end{tabular}

\section{* Chart continued below}

\begin{tabular}{|c|c|c|c|c|c|}
\hline $\begin{array}{l}\text { Küchler, A. } \\
\text { M. (2019). } \\
\text { Effectiveness } \\
\text { of an internet- } \\
\text { based } \\
\text { intervention } \\
\text { for } \\
\text { procrastinatio } \\
\text { n in college } \\
\text { students } \\
\text { (StudiCare } \\
\text { Procrastinatio } \\
\text { n): Study } \\
\text { protocol of a } \\
\text { randomized } \\
\text { controlled } \\
\text { trial. }\end{array}$ & $\begin{array}{l}\text { Germany and } \\
\text { Austria }\end{array}$ & $\begin{array}{l}\text { Two armed } \\
\text { randomized } \\
\text { controlled } \\
\text { trials } \\
(\mathrm{n}=120) \text {. } \\
\text { Intervention } \\
\text { group will } \\
\text { receive the e- } \\
\text { coach guided } \\
5 \text {-week IMI } \\
\text { StudiCare } \\
\text { procrastinatio } \\
\text { n and a } \\
\text { waitlist } \\
\text { control will } \\
\text { be given an } \\
\text { unguided IMI } \\
12 \text { weeks } \\
\text { after } \\
\text { randomizatio } \\
\text { n }\end{array}$ & $\begin{array}{l}\text { Questionnaire } \\
\text { s are used to } \\
\text { measure } \\
\text { irrational } \\
\text { procrastinatio } \\
\text { n, temptation, } \\
\text { depression, } \\
\text { anxiety, well } \\
\text { being and self } \\
\text { efficacy, and } \\
\text { client } \\
\text { satisfaction } \\
\text { was also } \\
\text { measured }\end{array}$ & $\begin{array}{l}\text { If it proves to } \\
\text { be effective, } \\
\text { StudiCare } \\
\text { Procrastinatio } \\
\text { n could } \\
\text { provide a } \\
\text { low- } \\
\text { threshold, } \\
\text { cost-efficient } \\
\text { way to help } \\
\text { the multitude } \\
\text { of students } \\
\text { suffering } \\
\text { from } \\
\text { problems } \\
\text { caused by } \\
\text { procrastinatio } \\
\text { n behavior. }\end{array}$ & $2 / * * *$ \\
\hline $\begin{array}{l}\text { Loeffler, S. } \\
\text { N. (2019). } \\
\text { Fostering } \\
\text { self- } \\
\text { regulation to } \\
\text { overcome } \\
\text { academic } \\
\text { procrastinatio } \\
\text { n using } \\
\text { interactive } \\
\text { ambulatory } \\
\text { assessment. }\end{array}$ & Germany & $\begin{array}{l}\text { Mixed } \\
\text { Methods } \\
\text { Design: A } \\
\text { total of } 98 \\
\text { volunteers } \\
\text { were } \\
\text { randomly } \\
\text { assigned to } \\
\text { the IG and } \\
\text { the CG but } \\
\text { were not told } \\
\text { to which } \\
\text { group they } \\
\text { belonged. }\end{array}$ & $\begin{array}{l}\text { In phase } 1 \\
\text { additional } \\
\text { interactive } \\
\text { features. } \\
\text { Within the } \\
\text { Morning } \\
\text { query, the IG } \\
\text { participants } \\
\text { obtained a } \\
\text { notice that a } \\
\text { feedback link } \\
\text { was available } \\
\text { through the } \\
\text { message }\end{array}$ & $\begin{array}{l}\text { Most existing } \\
\text { programs for } \\
\text { reducing } \\
\text { academic } \\
\text { procrastinatio } \\
\text { n involve } \\
\text { elaborate } \\
\text { face-to-face } \\
\text { training, and } \\
\text { most } \\
\text { interventions } \\
\text { do not take } \\
\text { place in real- } \\
\text { life situations }\end{array}$ & $2 / * * * *$ \\
\hline
\end{tabular}




\begin{tabular}{|c|c|c|c|c|c|}
\hline & & $\begin{array}{l}\text { Four } \\
\text { participants } \\
\text { prematurely } \\
\text { terminated } \\
\text { the study }\end{array}$ & $\begin{array}{l}\text { feature as of } \\
7: 30 \text { a.m. The } \\
\text { link provided } \\
\text { individualize } \\
\text { d feedback } \\
\text { with respect } \\
\text { to study and } \\
\text { procrastinatio } \\
\text { n behavior in } \\
\text { tabular form } \\
\text { presented on } \\
\text { the } \\
\text { smartphone } \\
\text { display. For } \\
\text { the Evening } \\
\text { query, the IG } \\
\text { was requested } \\
\text { to provide } \\
\text { reasons for } \\
\text { their } \\
\text { procrastinatio } \\
\text { n on the } \\
\text { respective } \\
\text { day. }\end{array}$ & $\begin{array}{l}\text { during the } \\
\text { preparation of } \\
\text { academic } \\
\text { deadlines. } \\
\text { Whereas the } \\
\text { contents of } \\
\text { our } \\
\text { interventions } \\
\text { are similar to } \\
\text { prior } \\
\text { trainings, our } \\
\text { approach } \\
\text { accentuates } \\
\text { the transfer of } \\
\text { new SR } \\
\text { strategies into } \\
\text { daily learning } \\
\text { routines by } \\
\text { using } \\
\text { intervening } \\
\text { elements } \\
\text { directly } \\
\text { during } \\
\text { preparation } \\
\text { for academic } \\
\text { deadlines. } \\
\text { The } \\
\text { individualize } \\
\text { d reason- } \\
\text { based } \\
\text { interventions } \\
\text { enabled the } \\
\text { students to } \\
\text { reduce } \\
\text { procrastinatio } \\
\text { n while } \\
\text { increasing } \\
\text { their } \\
\text { completed } \\
\text { workload and } \\
\text { effectively } \\
\text { using study } \\
\text { time. }\end{array}$ & \\
\hline $\begin{array}{l}\text { Malouff, J. } \\
\text { M.(2019). }\end{array}$ & Germany & $\begin{array}{l}\text { Meta } \\
\text { analysis; } 12\end{array}$ & $\begin{array}{l}12 \\
\text { randomized }\end{array}$ & $\begin{array}{l}\text { Significant } \\
\text { meta-analytic }\end{array}$ & $4 / * * * * * *$ \\
\hline
\end{tabular}




\begin{tabular}{|c|c|c|c|c|c|}
\hline $\begin{array}{l}\text { The Efficacy } \\
\text { of } \\
\text { Interventions } \\
\text { Aimed at } \\
\text { Reducing } \\
\text { Procrastinatio } \\
\mathrm{n} \text { : A } \\
\text { Meta } \square \text { Analys } \\
\text { is of } \\
\text { Randomized } \\
\text { Controlled } \\
\text { Trials. }\end{array}$ & & $\begin{array}{l}\text { studies with a } \\
\text { total of } 646 \\
\text { participants }\end{array}$ & $\begin{array}{l}\text { controlled } \\
\text { trials from six } \\
\text { different } \\
\text { countries. } \\
\text { Meta-analytic } \\
\text { effect, } \\
\text { confidence } \\
\text { intervals and } \\
\text { homogeneity } \\
\text { analysis were } \\
\text { done }\end{array}$ & $\begin{array}{l}\text { effect size } \\
\text { indicates that } \\
\text { the } \\
\text { interventions } \\
\text { had large } \\
\text { positive } \\
\text { effects. } \\
\text { Higher } \\
\text { effects were } \\
\text { associated } \\
\text { with in- } \\
\text { person } \\
\text { delivery of } \\
\text { intervention, } \\
\text { student } \\
\text { samples, and } \\
\text { a no } \\
\text { treatment } \\
\text { control }\end{array}$ & \\
\hline $\begin{array}{l}\text { Martinčeková } \\
\text {, L. (2020). } \\
\text { The effects of } \\
\text { self- } \\
\text { forgiveness } \\
\text { and shame- } \\
\text { proneness on } \\
\text { procrastinatio } \\
\text { n: exploring } \\
\text { the mediating } \\
\text { role of affect. }\end{array}$ & $\begin{array}{l}\text { Slovak } \\
\text { Republic }\end{array}$ & $\begin{array}{l}\text { University } \\
\text { students } \\
(\mathrm{n}=217) \\
\text { completed } \\
\text { online survey } \\
\text { which was } \\
\text { distributed } \\
\text { through their } \\
\text { email } \\
\text { addresses }\end{array}$ & $\begin{array}{l}\text { Online survey } \\
\text { asking about } \\
\text { demographic } \\
\text { information, } \\
\text { procrastinatio } \\
\text { n, self- } \\
\text { forgiveness } \\
\text { for } \\
\text { procrastinatio } \\
\text { n, shame- } \\
\text { proneness } \\
\text { and guilt } \\
\text { proneness, } \\
\text { and affect. } \\
\text { Correlational } \\
\text { analyses were } \\
\text { done }\end{array}$ & $\begin{array}{l}\text { Correlational } \\
\text { analyses } \\
\text { showed a } \\
\text { positive } \\
\text { relationship } \\
\text { between } \\
\text { procrastinatio } \\
\text { n with shame- } \\
\text { proneness } \\
\text { mediated } \\
\text { through the } \\
\text { presence of } \\
\text { negative } \\
\text { emotions but } \\
\text { no } \\
\text { relationship } \\
\text { between } \\
\text { procrastinatio } \\
\text { n with guilt } \\
\text { proneness. }\end{array}$ & $4 / * * * *$ \\
\hline $\begin{array}{l}\text { Rozental, A., } \\
\text { Forsell, E., } \\
\text { Svensson, A., } \\
\text { Forsström, } \\
\text { D., }\end{array}$ & Sweden & $\begin{array}{l}\text { Screening } \\
\text { process } \\
\text { recruited } \\
\text { participants } \\
(\mathrm{n}=710) \text { to a }\end{array}$ & $\begin{array}{l}\text { Self-report } \\
\text { measures of } \\
\text { procrastinatio } \\
\mathrm{n} \text {, depression, } \\
\text { anxiety, and }\end{array}$ & $\begin{array}{l}\text { The results } \\
\text { suggest there } \\
\text { exists five } \\
\text { separate } \\
\text { subgroups of }\end{array}$ & $4 / * * * * * *$ \\
\hline
\end{tabular}




\begin{tabular}{|c|c|c|c|c|c|}
\hline $\begin{array}{l}\text { Andersson, } \\
\text { G., \& } \\
\text { Carlbring, P. } \\
\text { (2015). } \\
\text { Differentiatin } \\
\text { g } \\
\text { procrastinator } \\
\text { s from each } \\
\text { other: A } \\
\text { cluster } \\
\text { analysis. }\end{array}$ & & $\begin{array}{l}\text { randomized } \\
\text { controlled } \\
\text { trial of } \\
\text { internet-based } \\
\text { cognitive } \\
\text { behavior } \\
\text { therapy for } \\
\text { procrastinatio } \\
\text { n }\end{array}$ & quality of life & $\begin{array}{l}\text { procrastinator } \\
\text { s in terms of } \\
\text { procrastinatio } \\
\text { n severity, } \\
\text { levels of } \\
\text { depression } \\
\text { and anxiety, } \\
\text { and quality of } \\
\text { life. Tailoring } \\
\text { intervention } \\
\text { to the type of } \\
\text { procrastinator } \\
\text { through a } \\
\text { screening } \\
\text { process might } \\
\text { be helpful }\end{array}$ & \\
\hline $\begin{array}{l}\text { Rozental, A.. } \\
\text { (2018). } \\
\text { Treating } \\
\text { procrastinatio } \\
\text { n using } \\
\text { cognitive } \\
\text { behavior } \\
\text { therapy: a } \\
\text { pragmatic } \\
\text { randomized } \\
\text { controlled } \\
\text { trial } \\
\text { comparing } \\
\text { treatment } \\
\text { delivered via } \\
\text { the internet or } \\
\text { in groups. }\end{array}$ & Sweden & $\begin{array}{l}\text { Pragmatic } \\
\text { randomized } \\
\text { controlled } \\
\text { trial } \\
\text { comparing } \\
\text { treatment } \\
\text { delivered } \\
\text { during } 8 \\
\text { weeks as self- } \\
\text { guided CBT } \\
\text { via the } \\
\text { internet or } \\
\text { group session } \\
\text { in university } \\
\text { students with } \\
\text { severe } \\
\text { procrastinatio } \\
\text { n (n=92(` }\end{array}$ & $\begin{array}{l}\text { Outcome } \\
\text { measures } \\
\text { related to } \\
\text { procrastinatio } \\
\text { n, depression, } \\
\text { anxiety and } \\
\text { well being } \\
\text { were done } \\
\text { before and } \\
\text { after } \\
\text { treatment and } \\
\text { at a 6-month } \\
\text { follow up. } \\
\text { Linear mixed } \\
\text { and fixed } \\
\text { effects } \\
\text { models, } \\
\text { improvement } \\
\text { and } \\
\text { deterioration } \\
\text { rates were } \\
\text { calculated }\end{array}$ & $\begin{array}{l}\text { No } \\
\text { differences } \\
\text { between } \\
\text { conditions } \\
\text { were } \\
\text { observed } \\
\text { after the } \\
\text { treatment } \\
\text { period but } \\
\text { those } \\
\text { participating } \\
\text { in group CBT } \\
\text { had longer } \\
\text { lasting } \\
\text { benefits at } \\
\text { follow up } \\
\text { than internet } \\
\text { CBT } \\
\text { participants. }\end{array}$ & $2 / * * * * *$ \\
\hline $\begin{array}{l}\text { Steel, } \\
\text { P.(2016). } \\
\text { Academic } \\
\text { procrastinatio } \\
\text { n: } \\
\text { Psychological }\end{array}$ & Australia & $\begin{array}{l}\text { University } \\
\text { students } \\
(\mathrm{n}=167) \\
\text { participating } \\
\text { in a } \\
\text { computerized }\end{array}$ & $\begin{array}{l}\text { Big Five } \\
\text { Inventory and } \\
\text { short essays } \\
\text { about reasons } \\
\text { for } \\
\text { procrastinatio }\end{array}$ & $\begin{array}{l}\text { Conscientious } \\
\text { ness and its } \\
\text { facets were } \\
\text { most strongly } \\
\text { correlated } \\
\text { with }\end{array}$ & $5 / * * * * * *$ \\
\hline
\end{tabular}




\begin{tabular}{|c|c|c|c|c|c|}
\hline $\begin{array}{l}\text { antecedents } \\
\text { revisited. }\end{array}$ & & $\begin{array}{l}\text { introductory } \\
\text { psychology } \\
\text { course } \\
\text { reported } \\
\text { about their } \\
\text { procrastinatio } \\
\text { n }\end{array}$ & $\begin{array}{l}\mathrm{n} \text { and } \\
\text { activities } \\
\text { pursued while } \\
\text { procrastinatin } \\
\mathrm{g} \text {. The } \\
\text { reasons and } \\
\text { activities } \\
\text { were coded } \\
\text { according to } \\
\text { their to the } \\
\text { personality } \\
\text { traits }\end{array}$ & $\begin{array}{l}\text { procrastinatio } \\
\text { n. Other } \\
\text { personality } \\
\text { traits did not } \\
\text { predict } \\
\text { procrastinatio } \\
\text { n tendencies. }\end{array}$ & \\
\hline $\begin{array}{l}\text { Vodanovich, } \\
\text { S. J., (1997). } \\
\text { Relationship } \\
\text { between time } \\
\text { structure and } \\
\text { procrastinatio } \\
\text { n. }\end{array}$ & Ireland & $\begin{array}{l}\text { University } \\
\text { students } \\
(\mathrm{n}=115) \text { self- } \\
\text { reported } \\
\text { measures } \\
\text { during their } \\
\text { regular class } \\
\text { time }\end{array}$ & $\begin{array}{l}\text { Self report } \\
\text { measures } \\
\text { using time } \\
\text { structure } \\
\text { questionnaire, } \\
\text { Tuckman } \\
\text { procrastinatio } \\
\text { n scale and } \\
\text { demographic } \\
\text { questions. } \\
\text { Variance and } \\
\text { regression } \\
\text { analyses were } \\
\text { done }\end{array}$ & $\begin{array}{l}\text { Significant } \\
\text { negative zero } \\
\text { order } \\
\text { correlations } \\
\text { were found } \\
\text { among all } \\
\text { five subscales } \\
\text { of time } \\
\text { structure and } \\
\text { procrastinatio } \\
\text { n. Significant } \\
\text { multiple } \\
\text { correlation } \\
\text { between all } \\
\text { five time } \\
\text { structure } \\
\text { subscale } \\
\text { scores and } \\
\text { procrastinatio } \\
\text { n }\end{array}$ & $4 / * * * * * *$ \\
\hline $\begin{array}{l}\text { Wessel, J., } \\
\text { Bradley, G. } \\
\text { L., \& Hood, } \\
\text { M. (2020). A } \\
\text { low } \square \text { intensit } \\
\text { y, } \\
\text { high } \square \text { frequen } \\
\text { cy } \\
\text { intervention } \\
\text { to reduce } \\
\text { procrastinatio } \\
\text { n. Applied }\end{array}$ & Australia & $\begin{array}{l}\text { Randomized } \\
\text { controlled } \\
\text { trial } \\
\text { University } \\
\text { students } \\
\text { (n=107) } \\
\text { participated } \\
\text { in } \\
\text { intervention } \\
\text { and n=315 } \\
\text { did not. } \\
\text { Completed }\end{array}$ & $\begin{array}{l}\text { Self report } \\
\text { measures of } \\
\text { progress } \\
\text { leading up to } \\
\text { an } \\
\text { assignment } \\
\text { and } \\
\text { procrastinatio } \\
\text { n, assignment } \\
\text { marks etc. }\end{array}$ & $\begin{array}{l}\text { Lower } \\
\text { behavioural } \\
\text { delay in the } \\
\text { intervention } \\
\text { condition } \\
\text { compared to } \\
\text { control } \\
\text { condition }\end{array}$ & $2 / * *$ \\
\hline
\end{tabular}




\begin{tabular}{|c|c|c|c|c|c|}
\hline Psychology. & & $\begin{array}{l}\text { baseline } \\
\text { questionnaire } \\
\text { and reported } \\
\text { their cell } \\
\text { phone } \\
\text { numbers. } \\
\text { They } \\
\text { received SMS } \\
\text { texts twice } \\
\text { daily for the } \\
\text { two weeks } \\
\text { leading up to } \\
\text { their } \\
\text { deadline. } \\
\text { Each SMS } \\
\text { included a } \\
\text { questionnaire } \\
\text { to indicate } \\
\text { progress. } \\
\text { Non- } \\
\text { participants } \\
\text { did not get an } \\
\text { SMS text }\end{array}$ & & & \\
\hline $\begin{array}{l}\text { Yile, D. } \\
(2020) \text {. } \\
\text { Characteristic } \\
\text { s and } \\
\text { intervention } \\
\text { of academic } \\
\text { procrastinatio } \\
\text { n of } \\
\text { students-An } \\
\text { analysis based } \\
\text { on } 1192 \\
\text { effective } \\
\text { samples of } \\
\text { Huaqiao } \\
\text { University. }\end{array}$ & China & $\begin{array}{l}\text { University } \\
\text { students } \\
(\mathrm{n}=1192) \\
\text { self-reported } \\
\text { procrastinatio } \\
\text { n measures }\end{array}$ & $\begin{array}{l}\text { Quantitative } \\
\text { descriptive } \\
\text { study: } \\
\text { questionnaire } \\
\text { s about } \\
\text { academic } \\
\text { procrastinatio } \\
\text { n, in } \\
\text { assignment, } \\
\text { exam } \\
\text { preparation, } \\
\text { and self } \\
\text { learning }\end{array}$ & $\begin{array}{l}52.4 \% \text { had } \\
\text { academic } \\
\text { procrastinatio } \\
\mathrm{n} ; 44.1 \% \text { had } \\
\text { procrastinatio } \\
\mathrm{n} \text { in } \\
\text { assignment } \\
\text { completion, } \\
61.2 \% \text { had } \\
\text { procrastinatio } \\
\text { n in exam } \\
\text { preparation, } \\
60.8 \% \text { had } \\
\text { procrastinatio } \\
\text { n in self } \\
\text { learning. }\end{array}$ & $4 / * * * *$ \\
\hline
\end{tabular}

\section{Appendix 3: Excluded Studies}




\begin{tabular}{|c|c|}
\hline Year & Title of Excluded Study \\
\hline 2017 & The role of academic procrastination as factor of university abandonment. \\
\hline 1983 & Procrastination: Why you do it, what to do about it. \\
\hline 1965 & Society and adolescent self-image. \\
\hline 2007 & "Rubicon model of action phases," \\
\hline 2012 & $\begin{array}{l}\text { An investigation into the self-handicapping behaviors of undergraduates in terms } \\
\text { of academic procrastination, the locus of control and academic success. }\end{array}$ \\
\hline 1906 & $\begin{array}{l}\text { Psychology: An introductory study of the structure and function of human } \\
\text { consciousness. }\end{array}$ \\
\hline 2005 & $\begin{array}{l}\text { Psychometric properties of the Spanish version of depression, anxiety and stress } \\
\text { scales. }\end{array}$ \\
\hline 1977 & Self-efficacy: toward a unifying theory of behavioral change. \\
\hline 2005 & $\begin{array}{l}\text { A closer look at the relationships among trait procrastination, neuroticism, and } \\
\text { conscientiousness. Personality and Individual Differences. }\end{array}$ \\
\hline 1993 & $\begin{array}{l}\text { A comparison of two measures of perfectionism. Personality and Individual } \\
\text { Differences. }\end{array}$ \\
\hline 1965 & $\begin{array}{l}\text { A rationale and test for the number of factors in factor analysis. Psychometrika, } \\
\text { v. } 30 \text {, p. } 179,1965 \text {, Horn J. L. }\end{array}$ \\
\hline 1984 & Academic procrastination: Frequency and cognitive-behavioral correlates. \\
\hline 1992 & $\begin{array}{l}\text { Academic procrastination: Personality correlates with Myers-Briggs types, self- } \\
\text { efficacy, and academic locus of control. }\end{array}$ \\
\hline 2016 & $\begin{array}{l}\text { Academic procrastination: Psychological antecedents revisited. Australian } \\
\text { Psychologist. }\end{array}$ \\
\hline 2017 & $\begin{array}{l}\text { Schemas mediate the link between procrastination and depression: Results from } \\
\text { the United States and Pakistan. }\end{array}$ \\
\hline
\end{tabular}




\begin{tabular}{|c|c|}
\hline 1982 & A personality profile of the college student procrastinator. \\
\hline 1991 & The theory of planned behavior. \\
\hline 1969 & The prediction of behavioral intentions in a choice situation. \\
\hline 1991 & Procrastination and obedience. \\
\hline 2006 & $\begin{array}{l}\text { An investigation of the academic procrastination among university students in } \\
\text { related to various variables. }\end{array}$ \\
\hline 2014 & $\begin{array}{l}\text { Advantages and limitations of Internet-based interventions for common mental } \\
\text { disorders. }\end{array}$ \\
\hline 2006 & Amos 7.0 user's guide. \\
\hline 1986 & At last, my research article on procrastination \\
\hline 2020 & $\begin{array}{l}\text { Pathologies associated with Problematic Internet Use. A systematic review and } \\
\text { meta-analysis in WoS and Scopus. }\end{array}$ \\
\hline 2006 & $\begin{array}{l}\text { The relationship between student teachers' procrastination behavior, decision } \\
\text { making and thinking styles. }\end{array}$ \\
\hline 2013 & $\begin{array}{l}\text { Academic procrastination, academic life satisfaction and academic achievement: } \\
\text { The mediation role of rational beliefs about studying. }\end{array}$ \\
\hline 2015 & $\begin{array}{l}\text { The moderator role of rational beliefs in relation to procrastination, academic } \\
\text { achievement and academic satisfaction. }\end{array}$ \\
\hline 2012 & $\begin{array}{l}\text { The direct and indirect role of self-esteem and procrastination in the relation to } \\
\text { fear of failure and self-worth. }\end{array}$ \\
\hline 2018 & $\begin{array}{l}\text { The protective role of rational beliefs on the relationship between irrational } \\
\text { beliefs, emotional states of stress, depression and anxiety. }\end{array}$ \\
\hline 2018 & $\begin{array}{l}\text { Procrastination, self-downing, self-doubt, and rational beliefs: A moderated } \\
\text { mediation model. }\end{array}$ \\
\hline 2018 & $\begin{array}{l}\text { Adolescent Internet abuse: A study on the role of attachment to parents and peers } \\
\text { in a large community sample. }\end{array}$ \\
\hline
\end{tabular}




\begin{tabular}{|l|l|}
1977 & Self-efficacy: toward a unifying theory of behavioral change. \\
\hline 2005 & The primacy of self-regulation in health promotion. \\
\hline 2010 & Psychological connectedness and intertemporal choice. \\
\hline 2011 & $\begin{array}{l}\text { On intertemporal selfishness: How the perceived instability of identity underlies } \\
\text { impatient consumption. }\end{array}$ \\
\hline 1997 & $\begin{array}{l}\text { Perspective taking: Imagining how another feels versus imagining how you } \\
\text { would feel. }\end{array}$ \\
\hline 1991 & Altruism in humans. \\
\hline 1996 & Self-regulation failure: An overview. \\
\hline 2007 & Self-regulation and the executive function: The self as controlling agent. \\
\hline 1996 & Manual for the beck depression inventory-II. \\
\hline 2000 & $\begin{array}{l}\text { Correlates and consequences of behavioral procrastination: the effects of } \\
\text { academic procrastination, self-consciousness, self-esteem and self-handicapping } \\
\text { (special issue). }\end{array}$ \\
\hline 2014 & Affect Regulation Training (ART). \\
\hline 1988 & $\begin{array}{l}\text { Emotion regulation and mental health: recent findings, current challenges, and } \\
\text { future directions. }\end{array}$ \\
\hline 2011 & $\begin{array}{l}\text { Psychological antecedents of student procrastination. } \\
\text { sample of iranian population. }\end{array}$ \\
\hline 1995 & A rational-emotive mental training program for professional athletes. \\
\hline 2015
\end{tabular}




\begin{tabular}{|l|l|}
2016 & $\begin{array}{l}\text { Procrastination, Distress and Life Satisfaction across the Age Range - A German } \\
\text { Representative Community Study }\end{array}$ \\
\hline 2013 & $\begin{array}{l}\text { Big five personality traits as a predictor of general and academic procrastination } \\
\text { behaviours among adolescents. }\end{array}$ \\
\hline 2008 & Autobiographical memory in childhood and the development of a continuous self. \\
\hline 2001 & $\begin{array}{l}\text { Imagining stereotypes away: The moderation of implicit stereotypes through } \\
\text { mental imagery. }\end{array}$ \\
\hline 1967 & Punctual and procrastinating students: A study of temporal parameters. \\
\hline 2015 & $\begin{array}{l}\text { Experiencing the temporally extended self: Initial support for the role of affective } \\
\text { states, vivid mental imagery, and future self } \square \text { continuity in the prediction of }\end{array}$ \\
\hline 2000 & $\begin{array}{l}\text { Task aversiveness and procrastination: a multi-dimensional approach to task } \\
\text { aversiveness across stages of personal projects. }\end{array}$ \\
\hline 1989 & Procrastination, busyness and bingeing. \\
\hline 2018 & $\begin{array}{l}\text { The relationships between problematic internet use, alexithymia levels and } \\
\text { attachment characteristics in a sample of adolescents in a high school, Turkey. }\end{array}$ \\
\hline 1993 & $\begin{array}{l}\text { Testing structural equation models. } \\
\text { davranişinın incelenmesi. }\end{array}$ \\
\hline 1997 & $\begin{array}{l}\text { Academic procrastination and irrational thinking: a reexamination with context } \\
\text { controlled. }\end{array}$ \\
\hline Helping students confront and deal with stress and procrastination. \\
\hline 2009 & $\begin{array}{l}\text { When will I succeed in my first-year diploma? Survival analysis in Dutch higher } \\
\text { education. }\end{array}$ \\
\hline Procrastination: why you do it and what to do about it. \\
\hline 2003
\end{tabular}




\begin{tabular}{|c|c|}
\hline 2006 & $\begin{array}{l}\text { Effects of dynamic and static imagery on vividness of imagery, skiing } \\
\text { performance, and confidence. }\end{array}$ \\
\hline 2003 & $\begin{array}{l}\text { Cognitive flexibility and adaptability to environmental changes in dynamic } \\
\text { complex problem } \square \text { solving tasks. }\end{array}$ \\
\hline 2015 & Internet addiction in university medical students. \\
\hline 2018 & $\begin{array}{l}\text { Problematic Use of the Internet and Smartphones in University Students: 2006- } \\
2017 .\end{array}$ \\
\hline 2006 & Mindfulness and self $\square$ acceptance. \\
\hline 2010 & Rational and irrational beliefs in primary prevention and mental health. \\
\hline 2018 & $\begin{array}{l}\text { Social anxiety, Internet and Cibersex addiction: its relationship with health } \\
\text { perception. }\end{array}$ \\
\hline 2019 & $\begin{array}{l}\text { A latent profile approach for the study of internet gaming disorder, social media } \\
\text { addiction, and psychopathology in a normative sample of adolescents. }\end{array}$ \\
\hline 2009 & $\begin{array}{l}\text { Eğitim fakültesi öğrencilerinin akademik erteleme davranışlarına ilişsin } \\
\text { görüşlerinin incelenmesi. }\end{array}$ \\
\hline 2010 & $\begin{array}{l}\text { Individual differences in undergraduate student athletes: the roles of } \\
\text { perfectionism and trait anxiety on perception of procrastination behavior. }\end{array}$ \\
\hline 1987 & Form, effect size, and power in moderated regression analysis. \\
\hline 2008 & Factor structure for Young's Internet Addiction Test: A confirmatory study. \\
\hline 1990 & The essentials of factor analysis ( $2 \mathrm{nd}$ ed.). \\
\hline 2010 & Internet addiction: a 21st century epidemic? \\
\hline 2009 & Adherence in internet interventions for anxiety and depression. \\
\hline 1997 & $\begin{array}{l}\text { Reinterpreting the empathy-altruism relationship: When one into one equals } \\
\text { oneness. }\end{array}$ \\
\hline
\end{tabular}




\begin{tabular}{|c|c|}
\hline 2018 & $\begin{array}{l}\text { A longitudinal study for the empirical validation of an etiopathogenetic model of } \\
\text { internet addiction in adolescence based on early emotion regulation. }\end{array}$ \\
\hline 2018 & $\begin{array}{l}\text { Relations between student procrastination and teaching styles: autonomy- } \\
\text { supportive and controlling. }\end{array}$ \\
\hline 1988 & Statistical power analysis for the behavioral sciences (2nd ed.). \\
\hline 2008 & $\begin{array}{l}\text { Reading ability as a predictor of academic procrastination among African } \\
\text { American graduate students. }\end{array}$ \\
\hline 2019 & Procrastination and Rational/Irrational Beliefs: A Moderated Mediation Model \\
\hline 1992 & Components of perfectionism and procrastination. \\
\hline 2002 & $\begin{array}{l}\text { Multidimensional fear of failure measurement: The performance failure appraisal } \\
\text { inventory. }\end{array}$ \\
\hline 2008 & Reinforcement sensitivity theory (RST): Introduction. \\
\hline 2013 & Approach and avoidance behavior: Multiple systems and their interactions. \\
\hline 2016 & $\begin{array}{l}\text { Reinforcement sensitivity theory of personality questionnaires: Structural survey } \\
\text { with recommendations. }\end{array}$ \\
\hline 2016 & $\begin{array}{l}\text { The reinforcement sensitivity theory of personality questionnaire (RST-PQ): } \\
\text { Development andvalidation. }\end{array}$ \\
\hline 1993 & Correlates of academic procrastination. \\
\hline 2014 & Trait approach motivation moderates the aftereffects of self-control. \\
\hline 2014 & Trait approach motivation moderates the aftereffects of self-control. \\
\hline 2011 & $\begin{array}{l}\text { Online interventions for social marketing health behavior change campaigns: a } \\
\text { meta-analysis of psychological architectures and adherence factors. }\end{array}$ \\
\hline 1986 & Self esteem in adolescents (Unpublished doctoral dissertation). \\
\hline 2010 & Self comes to mind: Constructing the conscious brain. \\
\hline
\end{tabular}




\begin{tabular}{|c|c|}
\hline 1999 & The feeling of what happens: Body and emotion in the making of consciousness. \\
\hline 2010 & $\begin{array}{l}\text { The role of rational and irrational beliefs in human functioning and disturbances: } \\
\text { Implications for research, theory, and clinical practice. }\end{array}$ \\
\hline 1980 & A multidimensional approach to individual differences in empathy. \\
\hline 1983 & $\begin{array}{l}\text { Measuring individual differences in empathy: Evidence for a multidimensional } \\
\text { approach. }\end{array}$ \\
\hline 2000 & Patterns of academic procrastination. \\
\hline 1997 & $\begin{array}{l}\text { Convergent and divergent validity of the beck anxiety inventory for patients with } \\
\text { panic disorder and agoraphobia. }\end{array}$ \\
\hline 2013 & Experiential avoidance and emotion regulation difficulties in hoarding disorder. \\
\hline 2004 & The functional architecture of human empathy. \\
\hline 1977 & Decision making: A psychological analysis of conflict, choice, and commitment. \\
\hline 1989 & Decisional procrastination: Examining personality correlates. \\
\hline 2007 & $\begin{array}{l}\text { Determining the number of factors to retain in EFA: An easy-to-use computer } \\
\text { program for carrying out parallel analysis. }\end{array}$ \\
\hline 2009 & $\begin{array}{l}\text { Determining the variables which explain the behavior of academic procrastination } \\
\text { in university students. }\end{array}$ \\
\hline 2002 & $\begin{array}{l}\text { Procrastination, temptations, and incentives: the struggle between the present and } \\
\text { the future in procrastinators and the punctual. }\end{array}$ \\
\hline 2018 & Problematic Internet use, maladaptive future time perspective and school context. \\
\hline 2002 & $\begin{array}{l}\text { Family Environment Variables Related to the Development of Failure-Avoidant } \\
\text { Patterns. }\end{array}$ \\
\hline 2018 & Mobile in Spain and in World 2018. \\
\hline
\end{tabular}




\begin{tabular}{|l|l|}
\hline 1998 & Do it now! Break the procrastination habit. \\
\hline 2012 & Does locus of control task interest have an effect on procrastination?. \\
\hline 2012 & Dealing with procrastination: The REBT approach and a demonstration session. \\
\hline 2008 & The fundamental of rational emotive behavior therapy. \\
\hline 2004 & Rational emotive behavioral counselling in action. \\
\hline 2006 & Historical aspects of mindfulness and self $\square$ acceptance in psychotherapy. \\
\hline 2014 & $\begin{array}{l}\text { The roles of academic procrastination tendency on the relationships among self- } \\
\text { doubt, self-esteem, and academic achievement. }\end{array}$ \\
\hline 1994 & $\begin{array}{l}\text { Dysfunctional procrastination and its relationship with self-esteem, interpersonal } \\
\text { dependency, and self-defeating behaviors. }\end{array}$ \\
\hline 2014 & $\begin{array}{l}\text { Efficacy and cost-effectiveness of minimal guided and unguided internet-based } \\
\text { mobile supported stress-management in employees with occupational stress: a } \\
\text { three-armed randomised controlled trial. }\end{array}$ \\
\hline 1997 & $\begin{array}{l}\text { Overcome procrastination: enhancing emotion regulation skills reduce } \\
\text { procrastination. }\end{array}$ \\
\hline 2016 & $\begin{array}{l}\text { Teachers' emotion regulation skills facilitate implementation of health-related } \\
\text { intentions. }\end{array}$ \\
\hline $\begin{array}{l}\text { The academic irrational beliefs scale: development, validation, and implications } \\
\text { for college counselors. }\end{array}$ \\
\hline 1962 & Overcoming Procrastination. \\
\hline 2003 & Discomfort anxiety: A new cognitive-behavioral construct (Part I). \\
\hline 1997
\end{tabular}




\begin{tabular}{|c|c|}
\hline 1997 & Overcoming procrastination. \\
\hline 2009 & $\begin{array}{l}\text { Don't stop thinking about tomorrow: Individual differences in future } \\
\text { self } \square \text { continuity account for saving. }\end{array}$ \\
\hline 2009 & $\begin{array}{l}\text { Saving for the future self: Neural measures of future self } \square \text { continuity predict } \\
\text { temporal discounting. }\end{array}$ \\
\hline 1993 & $\begin{array}{l}\text { Frontal lobe and frontal } \square \text { striatal substrates for different forms of human cognitive } \\
\text { flexibility. }\end{array}$ \\
\hline 1985 & $\begin{array}{l}\text { A Monte Carlo study of the effects of correlated method variance in moderated } \\
\text { multiple regression analysis. }\end{array}$ \\
\hline 2004 & $\begin{array}{l}\text { Maternal personality characteristics, affective state, and psychopathology in } \\
\text { relation to children's attention deficit and hyperactivity disorder and comorbid } \\
\text { symptoms (Unpublished master dissertation). }\end{array}$ \\
\hline 1997 & $\begin{array}{l}\text { Explaining lower-order traits through higher-order factors: The case of trait } \\
\text { procrastination, conscientiousness, and the specificity dilemma. }\end{array}$ \\
\hline 2000 & Procrastination: a means of avoiding shame or guilt? \\
\hline 2000 & Procrastination: A means of avoiding shame and guilt? \\
\hline 1997 & $\begin{array}{l}\text { The double-edged sword of self-handicapping: Discounting, augmentation, and } \\
\text { the protection and enhancement of self-esteem. }\end{array}$ \\
\hline 2015 & $\begin{array}{l}\text { Problematic internet use in university students: associated factors and differences } \\
\text { of gender. }\end{array}$ \\
\hline 2016 & $\begin{array}{l}\text { The contribution of metacognitions and attentional control to decisional } \\
\text { procrastination. }\end{array}$ \\
\hline 2008 & Metacognitions about procrastination: A preliminary investigation. \\
\hline 2017 & The Unintentional Procrastination Scale. \\
\hline 2009 & $\begin{array}{l}\text { Life regrets by avoidant and arousal procrastinators. Why put off today what you } \\
\text { will regret tomorrow? }\end{array}$ \\
\hline
\end{tabular}




\begin{tabular}{|l|l|}
2007 & Procrastination: different time orientations reflect different motives. \\
\hline 2000 & $\begin{array}{l}\text { Examining behavioral processes in indecision: decisional procrastination and } \\
\text { decision-making style }\end{array}$ \\
\hline 1995 & Procrastination and Task Avoidance: Theory, Research, and Treatment. \\
\hline 1995 & Procrastination and Task Avoidance: Theory, Research, and Treatment. \\
\hline 1994 & $\begin{array}{l}\text { Dysfunctional procrastination and its relationship with self-esteem, interpersonal } \\
\text { dependency, and self-defeating behaviors. }\end{array}$ \\
\hline 2000 & $\begin{array}{l}\text { Procrastination and attention: Factor analysis of attention defcit, boredomness, } \\
\text { intelligence, self-esteem, and task delay frequencies. }\end{array}$ \\
\hline 2007 & $\begin{array}{l}\text { Perceptions of self-concept and self-presentation by procrastinators: Further } \\
\text { evidence. }\end{array}$ \\
\hline 1994 & $\begin{array}{l}\text { Procrastination as revenge: do people report using delays as a strategy for } \\
\text { vengeance? }\end{array}$ \\
\hline 1997 & Exploring the time preferences of procrastinators: night or day, which is the one? \\
\hline 1995 & Procrastination and task avoidance: Theory, research, and treatment. \\
\hline 2007 & $\begin{array}{l}\text { Procrastination: Different time orientations reflect different motives. } \\
\text { procrastinatory cognitions inventory. }\end{array}$ \\
\hline 1995 & $\begin{array}{l}\text { Procrastination and task avoidance: theory, research, and treatment. } \\
\text { service. }\end{array}$ \\
\hline 1992 & Componenentination, negative self-judgments, and stress in depression and anxiety: A \\
\hline 2009
\end{tabular}




\begin{tabular}{|c|c|}
\hline 1995 & $\begin{array}{l}\text { Procrastination, negative self } \square \text { evaluation, and stress in depression and anxiety: A } \\
\text { review and preliminary model. }\end{array}$ \\
\hline 2014 & $\begin{array}{l}\text { Construct validity and factor structure of the difficulties in emotion regulation } \\
\text { scale among adults with severe mental illness. }\end{array}$ \\
\hline 1966 & Compliance without pressure: the foot-in-the-door technique. \\
\hline 2003 & Individual differences in academic procrastination tendency and writing success. \\
\hline 2005 & A Brief Introduction to Rational Emotive Behaviour Therapy (3rd Ed.) \\
\hline 2010 & Time management: Procrastination tendency in individual and collaborative tasks. \\
\hline 2018 & $\begin{array}{l}\text { Pilot study of a web-based acceptance and commitment therapy intervention for } \\
\text { university students to reduce academic procrastination. }\end{array}$ \\
\hline 2016 & $\begin{array}{l}\text { Adicción a internet en jóvenes y adultos pertenecientes a la Universidad Nacional } \\
\text { Autónoma de México. }\end{array}$ \\
\hline 2018 & $\begin{array}{l}\text { Internet addiction and procrastination among Chinese young adults: A moderated } \\
\text { mediation model. }\end{array}$ \\
\hline 2005 & $\begin{array}{l}\text { Psychometric properties of a Persian-language version of the beck depression } \\
\text { inventory-second edition: BDI-II-PERSIAN. }\end{array}$ \\
\hline 2012 & $\begin{array}{l}\text { Cultural adaptation of the difficulties in emotion regulation scale: reliability and } \\
\text { validity of an Italian version. }\end{array}$ \\
\hline 2015 & $\begin{array}{l}\text { An investigation of the efficacy of acceptance-based behavioral therapy for } \\
\text { academic procrastination. }\end{array}$ \\
\hline 2009 & $\begin{array}{l}\text { Behavioral self-regulation for weight loss in young adults: a randomized } \\
\text { controlled trial. }\end{array}$ \\
\hline 1995 & Internet addictive disorder (IAD) diagnostic criteria. \\
\hline 2018 & $\begin{array}{l}\text { The relationship between academic procrastination and metacognitive beliefs: [in } \\
\text { persian]. }\end{array}$ \\
\hline
\end{tabular}




\begin{tabular}{|c|c|}
\hline 1999 & Implementation intentions: Strong effects of simple plans. \\
\hline 2006 & $\begin{array}{l}\text { Implementation intentions and goal achievement: a meta-analysis of effects and } \\
\text { processes. }\end{array}$ \\
\hline 1999 & Implementation intentions: Strong effects of simple plans. \\
\hline 2013 & $\begin{array}{l}\text { The effect of the foot-in-the-door technique on sales in a computer-mediated field } \\
\text { setting. }\end{array}$ \\
\hline 2004 & $\begin{array}{l}\text { Multidimensional assessment of emotion regulation and dysregulation: } \\
\text { development, factor structure, and initial validation of the difficulties in emotion } \\
\text { regulation scale. }\end{array}$ \\
\hline 2000 & $\begin{array}{l}\text { The neuropsychology of anxiety: An enquiry into the functions of the Septo- } \\
\text { hippocampal System ( } 2 \text { nd ed.). }\end{array}$ \\
\hline 1984 & $\begin{array}{l}\text { A cognitive-behavioral assessment of problematic academic procrastination: } \\
\text { development of a procrastination self-statement inventory. }\end{array}$ \\
\hline 1980 & The totalitarian ego: Fabrication and revision of personal history. \\
\hline 1986 & $\begin{array}{l}\text { Modeling and mental imagery use by multiply handicapped and learning disabled } \\
\text { preschool children. }\end{array}$ \\
\hline 2015 & Handbook of Emotion Regulation, 2nd Edn. \\
\hline 2002 & Foot-in-the-door technique and computer mediated communication. \\
\hline 1999 & Sequential request strategy: effect on donor generosity. \\
\hline 2008 & Foot-in-the-door technique using a courtship request: a field experiment. \\
\hline 2014 & $\begin{array}{l}\text { Genetic relations among procrastination, impulsivity, and goal } \square \text { management } \\
\text { ability: Implications for the evolutionary origin of procrastination. }\end{array}$ \\
\hline 2015 & $\begin{array}{l}\text { Conceptualization and operationalization of delay: Development and validation of } \\
\text { the multifaceted measure of academic procrastination and the delay questionnaire } \\
\text { (doctoral dissertation). }\end{array}$ \\
\hline
\end{tabular}




\begin{tabular}{|c|c|}
\hline 2012 & The complexity of the relation between fear of failure and procrastination. \\
\hline 2018 & $\begin{array}{l}\text { Psychometric properties of the difficulties in emotion regulation scale (DERS) } \\
\text { and its short forms in adults with emotional disorders. }\end{array}$ \\
\hline 2005 & It's too difficult! Frustration intolerance beliefs and procrastination. \\
\hline 1996 & Prevalence of procrastination among samples of adults. \\
\hline 1996 & $\begin{array}{l}\text { Distractibility, daydreaming, and self-critical cognitions as determinants of } \\
\text { indecision. }\end{array}$ \\
\hline 1996 & Prevalence of procrastination among samples of adults. \\
\hline 1998 & Procrastination in college students: the role of self efficacy and anxiety. \\
\hline 2013 & $\begin{array}{l}\text { Introduction to mediation, moderation, and conditional process analysis: A } \\
\text { regression-based approach. }\end{array}$ \\
\hline 2014 & Statistical mediation analysis with a multicategorical independent variable. \\
\hline 1987 & $\begin{array}{l}\text { Thought contents and cognitive functioning in motivational vs. volitional states of } \\
\text { mind. }\end{array}$ \\
\hline 2011 & $\begin{array}{l}\text { Short horizons and tempting situations: Lack of continuity to our future selves } \\
\text { leads to unethical decision making and behavior. }\end{array}$ \\
\hline 2011 & Increasing saving behavior through age $\square$ progressed renderings of the future self. \\
\hline 2018 & $\begin{array}{l}\text { The efficacy of group acceptance and commitment therapy on reducing academic } \\
\text { procrastination and improving difficulty in emotion regulation: a randomized } \\
\text { clinical trial. }\end{array}$ \\
\hline 2013 & Prokrastination: Ein Manual zur Behandlung des Pathologischen Aufschiebens. \\
\hline 2010 & Mental imagery in emotion and emotional disorders. \\
\hline 2008 & $\begin{array}{l}\text { Looking at or through rose } \square \text { tinted glasses? Imagery perspective and positive } \\
\text { mood. }\end{array}$ \\
\hline
\end{tabular}




\begin{tabular}{|l|l|}
2006 & $\begin{array}{l}\text { Positive interpretation training: Effects of mental imagery versus verbal training } \\
\text { on positive mood. }\end{array}$ \\
\hline 2006 & Academic procrastination. The pattern and correlates of behavioral postponement. \\
\hline 2007 & $\begin{array}{l}\text { Procrastination: associations with achievement goal orientation and learning } \\
\text { strategies. B211 }\end{array}$ \\
\hline 2005 & $\begin{array}{l}\text { The relationship of motivation and flow experience to academic procrastination in } \\
\text { university students }\end{array}$ \\
\hline 2006 & $\begin{array}{l}\text { [The relationship study on the relationship between procrastination behaviors and } \\
\text { bad personality disposition] }\end{array}$ \\
\hline 2008 & $\begin{array}{l}\text { College students who have an eveningness preference report lower self-control } \\
\text { and greater procrastination }\end{array}$ \\
\hline 2012 & $\begin{array}{l}\text { Cognitive behavior therapy via the Internet: a systematic review of applications, } \\
\text { clinical efficacy and cost-effectiveness }\end{array}$ \\
\hline 2013 & $\begin{array}{l}\text { Relationship between procrastination and academic performance among a group } \\
\text { of undergraduate dental students in India }\end{array}$ \\
\hline 2014 & $\begin{array}{l}\text { Internet-based cognitive behavioral therapy for patients with chronic somatic } \\
\text { conditions: a meta-analytic review }\end{array}$ \\
\hline 2014 & $\begin{array}{l}\text { Internet-vs. group-delivered cognitive behavior therapy for insomnia: A } \\
\text { randomized controlled non-inferiority trial }\end{array}$ \\
\hline $\begin{array}{l}\text { Traumatized refugees: morbidity, treatment and predictors of outcome } \\
\text { the irrational procrastination scale, and the susceptibility to temptation scale in a } \\
\text { clinical population }\end{array}$ \\
\hline $\begin{array}{l}\text { Internet-based cognitive-behavior therapy for procrastination: A randomized } \\
\text { controlled trial }\end{array}$ \\
\hline 2016 & $\begin{array}{l}\text { Internet-based cognitive-behavior therapy for procrastination: A randomized } \\
\text { controlled trial }\end{array}$ \\
\hline 2015
\end{tabular}




\begin{tabular}{|l|l|}
2017 & $\begin{array}{l}\text { Overcoming procrastination: one-year follow-up and predictors of change in a } \\
\text { randomized controlled trial of Internet-based cognitive behavior therapy }\end{array}$ \\
\hline 2016 & Prevalence of problematic internet use in Slovenia \\
\hline 2017 & $\begin{array}{l}\text { A Brazilian Investigation of the 36- and 16-Item Difficulties in Emotion } \\
\text { Regulation Scales }\end{array}$ \\
\hline 2018 & Academic interventions for academic procrastination: A review of the literature \\
\hline 2018 & Academic interventions for academic procrastination: A review of the literature \\
\hline 2018 & Academic interventions for academic procrastination: A review of the literature \\
\hline 2018 & Academic interventions for academic procrastination: A review of the literature \\
\hline 2018 & $\begin{array}{l}\text { Roles of Impulsivity, Motivation, and Emotion Regulation in Procrastination - } \\
\text { Path Analysis and Comparison Between Students and Non-students }\end{array}$ \\
\hline 2018 & Hoarding Disorder and Difficulties in Emotion Regulation \\
\hline 2020 & $\begin{array}{l}\text { Contributions of Positive Psychology in Self-Regulated Learning: A Study With } \\
\text { Brazilian Undergraduate Students }\end{array}$ \\
\hline 2020 & $\begin{array}{l}\text { Academic procrastination in nursing students. Spanish adaptation of the } \\
\text { Academic Procrastination Scale-Short Form (APS-SF) }\end{array}$ \\
\hline 2020 & $\begin{array}{l}\text { Mexican and Spanish university students' Internet addiction and academic } \\
\text { procrastination: Correlation and potential factors }\end{array}$ \\
\hline $\begin{array}{l}\text { State and Trait Anxiety Among University Students: A Moderated Mediation } \\
\text { Model of Negative Affectivity, Alexithymia, and Housing Conditions }\end{array}$ \\
\hline $\begin{array}{l}\text { Uncovering the Moderating Role of Grit and Gender in the Association between } \\
\text { Undergraduate Students }\end{array}$ \\
\hline features
\end{tabular}




\begin{tabular}{|l|l|}
2020 & $\begin{array}{l}\text { A Structural Equation Model of Perceived Autonomy Support and Growth } \\
\text { Mindset in Undergraduate Students: The Mediating Role of Sense of Coherence }\end{array}$ \\
\hline 2020 & $\begin{array}{l}\text { Parental Attachment and Problematic Internet Use among Chinese Adolescents: } \\
\text { The Moderating Role of Gender and Grit }\end{array}$ \\
\hline 2021 & $\begin{array}{l}\text { COVID-19 Infection Risk and Depressive Symptoms Among Young Adults } \\
\text { During Quarantine: The Moderating Role of Grit and Social Support }\end{array}$ \\
\hline 2018 & $\begin{array}{l}\text { Does SMS-Support Make a Difference? Effectiveness of a Two-Week Online- } \\
\text { Training to Overcome Procrastination. A Randomized Controlled Trial }\end{array}$ \\
\hline 2018 & $\begin{array}{l}\text { Efficacy of a guided internet-based intervention (iSOMA) for somatic symptoms } \\
\text { and related distress in university students: study protocol of a randomised } \\
\text { controlled trial }\end{array}$ \\
\hline 2019 & $\begin{array}{l}\text { Effectiveness of an internet-based intervention for procrastination in college } \\
\text { students (StudiCare Procrastination): Study protocol of a randomized controlled } \\
\text { trial }\end{array}$ \\
\hline 2014 & $\begin{array}{l}\text { Absorbed in the moment? An investigation of procrastination, absorption and } \\
\text { cognitive failures }\end{array}$ \\
\hline 2015 & $\begin{array}{l}\text { The mystery of "should": Procrastination, delay, and reactance in academic } \\
\text { settings }\end{array}$ \\
\hline 2015 & $\begin{array}{l}\text { Cognitive, emotional, and motivational factors related to procrastination: A } \\
\text { cluster analytic approach } \\
\text { married }\end{array}$ \\
\hline Procrastination as a self-regulation failure: The role of inhibition, negative affect, \\
and gender $\square$
\end{tabular}




\begin{tabular}{|c|c|}
\hline 2018 & $\begin{array}{l}\text { Backward and forward serial recall across modalities: An individual differences } \\
\text { perspective }\end{array}$ \\
\hline 2016 & Problematic Facebook use and procrastination \\
\hline 2016 & $\begin{array}{l}\text { Procrastination and self-efficacy: Tracing vicious and virtuous circles in self- } \\
\text { regulated learning }\end{array}$ \\
\hline 2011 & $\begin{array}{l}\text { Comparing active delay and procrastination from a self-regulated learning } \\
\text { perspective }\end{array}$ \\
\hline 2013 & Children's additive concepts: Promoting understanding and the role of inhibition \\
\hline 2013 & $\begin{array}{l}\text { Motivated or paralyzed? Individuals' beliefs about intelligence influence } \\
\text { performance outcome of expecting rapid feedback }\end{array}$ \\
\hline 2013 & $\begin{array}{l}\text { Considering word characteristics for spelling accuracy: Evidence from Korean- } \\
\text { speaking children }\end{array}$ \\
\hline 2013 & $\begin{array}{l}\text { The role of lexical representations and phonological overlap in rhyme judgments } \\
\text { of beginning, intermediate and advanced readers }\end{array}$ \\
\hline 2013 & The role of explicit need strength for emotions during learning \\
\hline 2013 & $\begin{array}{l}\text { Extending antecedents of achievement goals: The double-edged sword effect of } \\
\text { social-oriented achievement motive and gender differences }\end{array}$ \\
\hline 2014 & $\begin{array}{l}\text { Reconsidering active procrastination: Relations to motivation and achievement in } \\
\text { college anatomy }\end{array}$ \\
\hline 2019 & $\begin{array}{l}\text { Emergency department ergonomic design evaluation: A case study using fuzzy } \\
\text { DEMATEL-focused two-stage methodology }\end{array}$ \\
\hline 2019 & Biases and imperatives in handling medical technology \\
\hline 2014 & $\begin{array}{l}\text { A preliminary investigation of the role of psychological inflexibility in academic } \\
\text { procrastination }\end{array}$ \\
\hline 2016 & $\begin{array}{l}\text { Psychometric properties of the Avoidance and Fusion Questionnaire for Youth: A } \\
\text { psychological measure of psychological inflexibility in youth }\end{array}$ \\
\hline
\end{tabular}




\begin{tabular}{|c|c|}
\hline 2019 & $\begin{array}{l}\text { Assessing psychological inflexibility in university students: Development and } \\
\text { validation of the acceptance and action questionnaire for university students } \\
\text { (AAQ-US) }\end{array}$ \\
\hline 2019 & $\begin{array}{l}\text { The role of present moment awareness and cognitive fusion with food craving in } \\
\text { the relationship between depression and binge eating }\end{array}$ \\
\hline 2019 & $\begin{array}{l}\text { Treatment sensitivity: Its importance in the measurement of psychological } \\
\text { flexibility }\end{array}$ \\
\hline 2019 & $\begin{array}{l}\text { A Service Member's experience of Acceptance and Commitment Therapy for } \\
\text { Moral Injury (ACT-MI) via telehealth: "Learning to accept my pain and injury by } \\
\text { reconnecting with my values and starting to live a meaningful life" }\end{array}$ \\
\hline 2006 & Internet-based exercise intervention systems: Are more interactive designs better? \\
\hline 2014 & $\begin{array}{l}\text { The organization of irrational beliefs in posttraumatic stress symptomology: } \\
\text { Testing the predictions of REBT theory using structural equation modelling. }\end{array}$ \\
\hline 1997 & Empathic accuracy. \\
\hline 2017 & $\begin{array}{l}\text { Predictive Roles of Depression and Demographic Factors in Internet Addiction: A } \\
\text { Cross-Sectional Study of Students in a Nigerian University. }\end{array}$ \\
\hline 2001 & $\begin{array}{l}\text { The impact of hope, procrastination and social activity on academic performance } \\
\text { of Midwestern college students. }\end{array}$ \\
\hline 1085 & Psychology: The briefer course. \\
\hline 2013 & $\begin{array}{l}\text { Potentiating empathic growth: Generating imagery while reading fiction increases } \\
\text { empathy and prosocial behavior. }\end{array}$ \\
\hline 1995 & $\begin{array}{l}\text { An analysis of the contribution of the five factors of personality to variance in } \\
\text { academic procrastination. }\end{array}$ \\
\hline 1995 & $\begin{array}{l}\text { An analysis of the contribution of the fve factors of personality to variance in } \\
\text { academic procrastination. }\end{array}$ \\
\hline 1994 & Wherever you go, there you are: Mindfulness meditation in everyday life. \\
\hline
\end{tabular}




\begin{tabular}{|c|c|}
\hline 2016 & Pathological internet use is on the rise among european adolescents. \\
\hline 1979 & Intuitive prediction: biases and corrective procedures. \\
\hline 2015 & $\begin{array}{l}\text { A randomized controlled behavioral intervention trial to improve medication } \\
\text { adherence in adult stroke patients with prescription tailored Short Messaging } \\
\text { Service (SMS)-SMS4Stroke study. }\end{array}$ \\
\hline 1998 & Internet addiction on campus: The vulnerability of college students. \\
\hline 2012 & $\begin{array}{l}\text { Adaptive persuasive systems: a study of tailored persuasive text messages to } \\
\text { reduce snacking. }\end{array}$ \\
\hline 2004 & Tax Procrastination: Survey Finds 29\% have yet to begin Taxes. \\
\hline 1983 & What does it mean to be a high imager? \\
\hline 2011 & Enhanced active choice: a new method to motivate behavior change. \\
\hline 2019 & $\begin{array}{l}\text { Reductions in transdiagnostic factors as the potential mechanisms of change in } \\
\text { treatment outcomes in the unified protocol: a randomized clinical trial. }\end{array}$ \\
\hline 2012 & $\begin{array}{l}\text { Common and distinct neural mechanisms of attentional switching and response } \\
\text { conflict. }\end{array}$ \\
\hline 2015 & $\begin{array}{l}\text { The relationship between procrastination and academic performance: A meta- } \\
\text { analysis. }\end{array}$ \\
\hline 2006 & $\begin{array}{l}\text { The dark side of internet: Preliminary evidence for the associations of dark } \\
\text { personality traits with specific online activities and problematic internet use. }\end{array}$ \\
\hline 2012 & $\begin{array}{l}\text { The relationship between locus of control and perfectionism perception of the } \\
\text { primary school administrators (Unpublished doctorate dissertation). }\end{array}$ \\
\hline 2008 & $\begin{array}{l}\text { Academic procrastination of undergraduates: low self-efficacy to self-regulate } \\
\text { predicts higher levels of procrastination. }\end{array}$ \\
\hline 2008 & $\begin{array}{l}\text { Academic procrastination of undergraduates: low self-efficacy to self-regulate } \\
\text { predicts higher levels of procrastination. }\end{array}$ \\
\hline 2009 & Academic procrastination and motivation of adolescents in Turkey. \\
\hline
\end{tabular}




\begin{tabular}{|l|l|}
2008 & $\begin{array}{l}\text { Academic procrastination of undergraduates: Low self-efficacy to selfregulate } \\
\text { predicts higher levels of procrastination. }\end{array}$ \\
\hline 2008 & $\begin{array}{l}\text { Academic procrastination of undergraduates: Low self } \square \text { efficacy to self } \square \text { regulate } \\
\text { predicts higher levels of procrastination. }\end{array}$ \\
\hline 2005 & Principles and practice of structural equation modeling (2nd ed.). \\
\hline 2005 & Psychological testing: A practical approach to design and evaluation. \\
\hline 2013 & Procrastination. When good things don’t come to those who wait. \\
\hline 2012 & $\begin{array}{l}\text { Procrastination: development and validation of a German short scale of the } \\
\text { general procrastination scale. }\end{array}$ \\
\hline 2013 & Why students procrastinate: a qualitative approach. \\
\hline 2013 & When good things don’t come to those who wait. \\
\hline 2006 & Frustration tolerance training for children. \\
\hline 2009 & The psychology of emotion regulation: an integrative review. \\
\hline 2008 & $\begin{array}{l}\text { Effectiveness of mobile-phone short message service (SMS) reminders for } \\
\text { ophthalmology outpatient appointments: observational study. }\end{array}$ \\
\hline 2001 & Neural foundations of imagery. \\
\hline 2010 & The future of emotion research in the study of psychopathology. \\
\hline 2009 & Healthcare via cell phones: a systematic review. \\
\hline 1996 & Amagery techniques in the work of Mike and Nancy Samuels. \\
\hline At last, my research article on procrastination.. \\
\hline 2002
\end{tabular}




\begin{tabular}{|c|c|}
\hline 1986 & At last, my research article on procrastination. \\
\hline 1997 & $\begin{array}{l}\text { Conscientiousness, procrastination, and person-task characteristics in job } \\
\text { searching by unemployed adults. }\end{array}$ \\
\hline 1991 & $\begin{array}{l}\text { Intentions and behavior in studying for an examination: the role of trait } \\
\text { procrastination and its interaction with optimism. }\end{array}$ \\
\hline 1993 & Trait procrastination, time management, and academic behavior. \\
\hline 2005 & $\begin{array}{l}\text { The relationship of motivation and fow experience to academic procrastination in } \\
\text { university students. }\end{array}$ \\
\hline 2016 & $\begin{array}{l}\text { Addictive online games: Examining the relationship between game genres and } \\
\text { internet gaming disorder. }\end{array}$ \\
\hline 2015 & $\begin{array}{l}\text { When does the future begin? Time metrics matter, connecting present and future } \\
\text { selves. }\end{array}$ \\
\hline 2011 & $\begin{array}{l}\text { Visual perspective in mental imagery: A representational tool that functions in } \\
\text { judgment, emotion, and self } \square \text { insight. }\end{array}$ \\
\hline 2003 & Is attention to feelings beneficial or detrimental to affective well-being? \\
\hline 2020 & $\begin{array}{l}\text { Academic self-efficacy and postgraduate procrastination: a moderated mediation } \\
\text { model. }\end{array}$ \\
\hline 2013 & $\begin{array}{l}\text { Using mental imagery to deliver self } \square \text { regulation techniques to improve sleep } \\
\text { behaviors. }\end{array}$ \\
\hline 2016 & Traits associated with internet addiction in young adults: Potential risk factors. \\
\hline 2008 & $\begin{array}{l}\text { Phantom limb pain, cortical reorganization and the therapeutic effect of mental } \\
\text { imagery. }\end{array}$ \\
\hline 2011 & Cortical reorganization, mental imagery and pain. \\
\hline 2012 & $\begin{array}{l}\text { Trait procrastination among dental students in India and its influence on academic } \\
\text { performance. }\end{array}$ \\
\hline
\end{tabular}




\begin{tabular}{|c|c|}
\hline 2019 & $\begin{array}{l}\text { Problematic internet use in Bangladeshi students: The role of sociodemographic } \\
\text { factors, depression, anxiety, and stress. }\end{array}$ \\
\hline 1970 & Measures of multivariate skewness and kurtosis with applications. \\
\hline 1973 & Visual imagery differences in the recall of pictures. \\
\hline 1987 & Resolving the unvividness paradox. \\
\hline 2003 & $\begin{array}{l}\text { Self } \square \text { handicapping and defensive pessimism: A model of self } \square \text { protection from a } \\
\text { longitudinal perspective. }\end{array}$ \\
\hline 2015 & Abaris Financial and the revolution in retirement spending. \\
\hline 2011 & Finally, my thesis on academic procrastination (Master's thesis). \\
\hline 2015 & Finally! The development and validation of the Academic Procrastination Scale. \\
\hline 2012 & Content analyses of the beliefs of academic procrastinators. \\
\hline 1995 & $\begin{array}{l}\text { The VVIQ as a psychometric test of individual differences in visual imagery } \\
\text { vividness: A critical quantitative review and plea for direction. }\end{array}$ \\
\hline 2007 & $\begin{array}{l}\text { Delineating components of emotion and its dysregulation in anxiety and mood } \\
\text { psychopathology. }\end{array}$ \\
\hline 2006 & $\begin{array}{l}\text { Effects of guided imagery on outcomes of pain, functional status, and } \\
\text { self } \square \text { efficacy in persons diagnosed with fibromyalgia. }\end{array}$ \\
\hline 2019 & $\begin{array}{l}\text { Mexican Internet Association. } 15^{\circ} \text { study on the habits of internet users in Mexico } \\
2018 .\end{array}$ \\
\hline 1999 & $\begin{array}{l}\text { Academic anxiety, academic procrastination, and parental involvement in } \\
\text { students and their parents. }\end{array}$ \\
\hline 2018 & Action plan on addictions $2018-2020$. \\
\hline 2014 & $\begin{array}{l}\text { The role of positive and negative perfectionism, self-efficacy, worry and emotion } \\
\text { regulation in predicting behavioral and decisional procrastination. }\end{array}$ \\
\hline
\end{tabular}




\begin{tabular}{|l|l|}
2017 & $\begin{array}{l}\text { Comparison of anxiety and depression among students with and without } \\
\text { academic procrastination. }\end{array}$ \\
\hline 2018 & $\begin{array}{l}\text { Prevalence of academic procrastination and its association with metacognitive } \\
\text { beliefs in Zanjan University of medical sciences. }\end{array}$ \\
\hline 2009 & Meditation, mindfulness and cognitive flexibility. \\
\hline 2013 & Imagination and the self. \\
\hline 2007 & $\begin{array}{l}\text { The role of irrational beliefs and aggression on academic performance of grade } \\
\text { male students in Tabriz high schools. }\end{array}$ \\
\hline 2016 & $\begin{array}{l}\text { Procrastination, distress and life satisfaction across the age range: A German } \\
\text { representative community study. }\end{array}$ \\
\hline 1994 & Psychological reactance and brief treatment of academic procrastination. \\
\hline 1973 & Multidimensional locus of control in psychiatric patients. \\
\hline 2014 & $\begin{array}{l}\text { Exploring internet addiction, academic procrastination and general } \\
\text { procrastination among pre-service ICT teachers. }\end{array}$ \\
\hline 2001 & $\begin{array}{l}\text { The structure of common emotion regulation strategies: a meta-analytic } \\
\text { examination. }\end{array}$ \\
\hline 2012 & $\begin{array}{l}\text { On the social nature of personality: Effects of extraversion, agreeableness, and } \\
\text { feedback about collective resource use on cooperation in a resource dilemma. }\end{array}$ \\
\hline 2001 & $\begin{array}{l}\text { Mental practice is modifiable: Changes in perspective maintenance and vividness } \\
\text { post } \square \text { stroke. }\end{array}$ \\
\hline Incentives for procrastinators. \\
\hline Choice and procrastination. \\
\hline 2000
\end{tabular}




\begin{tabular}{|l|l|}
2000 & Academic procrastinators and perfectionistic tendencies among graduate students. \\
\hline 1998 & The causes and consequences of academic procrastination: A research note. \\
\hline 2017 & $\begin{array}{l}\text { Factor structure of the difficulties in emotion regulation scale (DERS) in adult } \\
\text { outpatients receiving dialectical behavior therapy (DBT). }\end{array}$ \\
\hline 1977 & Overcoming procrastination. \\
\hline 2008 & Overcoming procrastination: the effect of implementation intentions. \\
\hline 1997 & Procrastination in high school achievement: a causal structural model. \\
\hline 2011 & $\begin{array}{l}\text { Üniversite ögrencilerinin akademik erteleme nedenler [The reasons for academic } \\
\text { procrastination among university students]. }\end{array}$ \\
\hline 2009 & $\begin{array}{l}\text { Exploring academic procrastination among Turkish students: Possible gender } \\
\text { diferences in prevalence and reasons. }\end{array}$ \\
\hline 2009 & $\begin{array}{l}\text { Exploring academic procrastination among Turkish students: Possible gender } \\
\text { differences in prevalence and reasons. }\end{array}$ \\
\hline 2009 & $\begin{array}{l}\text { Exploring academic procrastination among Turkish students: possible gender } \\
\text { differences in prevalence and reasons. }\end{array}$ \\
\hline 1994 & Parental authority and the development of female dysfunctional procrastination. \\
\hline 1971 & Personal identity. \\
\hline 2016 & $\begin{array}{l}\text { Fear and anxiety as separable emotions: An investigation of the revised } \\
\text { reinforcement sensitivity theory of personality. }\end{array}$ \\
\hline 2007 & Addiction and factors of Internet misuse, in a sample of university students. \\
\hline 2012
\end{tabular}




\begin{tabular}{|l|l|}
1996 & $\begin{array}{l}\text { Personality correlates of depression and health symptoms: A test of a self- } \\
\text { regulation model. }\end{array}$ \\
\hline 2005 & Personality in adulthood: A five-factor theory perspective. \\
\hline 2009 & How do emotion and motivation direct executive control? \\
\hline 2017 & Mobile abuse in university students and profiles of victimization and aggression. \\
\hline 2007 & Self-criticism, goal motivation, and goal progress. \\
\hline 2011 & $\begin{array}{l}\text { Efect size measures for mediation models: Quantitative strategies for } \\
\text { communicating indirect efects. }\end{array}$ \\
\hline 2014 & Predictability of personality traits on the procrastination behaviour. \\
\hline 2009 & $\begin{array}{l}\text { Prevalence of academic procrastination behavior among pre-service teachers, and } \\
\text { its relationships with demographics and individual preferences. }\end{array}$ \\
\hline 1996 & Prevalence of procrastination among samples of adults. \\
\hline 1995 & Procrastination and task avoidance: Theory, research, and treatment. \\
\hline 1998 & Procrastination in college students: The role of self-efficacy and anxiety. \\
\hline 2013 & $\begin{array}{l}\text { Procrastination in different life-domains: Is procrastination domain specific?. } \\
\text { a function of motivation toward college work. }\end{array}$ \\
\hline 2013 & Procrastination when good things don't come to those who wait. \\
\hline 2011 & $\begin{array}{l}\text { Psychometric properties of the Turkish version of the Frost Multidimensional } \\
\text { Perfectionism Scale. }\end{array}$ \\
\hline 2013 & Problematic Internet use in a sample of Colombian university students. \\
\hline 1992
\end{tabular}




\begin{tabular}{|c|c|}
\hline 2012 & Procrastination and self-regulatory failure: an introduction to the special issue. \\
\hline 2000 & $\begin{array}{l}\text { Five days of emotion: an experience sampling study of undergraduate student } \\
\text { procrastination. }\end{array}$ \\
\hline 2016 & Procrastination, emotion regulation, and well-being \\
\hline 2002 & $\begin{array}{l}\text { Parenting and procrastination: Gender diferences in the relations between } \\
\text { procrastination, parenting style and self-worth in early adolescence. }\end{array}$ \\
\hline 2000 & $\begin{array}{l}\text { Five days of emotion: An experience sampling study of undergraduate student } \\
\text { procrastination. }\end{array}$ \\
\hline 2004 & A project $\square$ analytic perspective on academic procrastination and intervention. \\
\hline 2013 & $\begin{array}{l}\text { An investigation into the reliability and validity of beck anxiety inventory among } \\
\text { the university students. }\end{array}$ \\
\hline 2014 & $\begin{array}{l}\text { Reasons of academic procrastination: Self-regulation, academic self-efficacy, life } \\
\text { satisfaction and demographics variables. }\end{array}$ \\
\hline 2018 & $\begin{array}{l}\text { Procrastination as a self-regulation failure: the role of impulsivity and intrusive } \\
\text { thoughts. }\end{array}$ \\
\hline 2015 & $\begin{array}{l}\text { Cognitive, emotional, and motivational factors related to procrastination: a cluster } \\
\text { analytic approach. }\end{array}$ \\
\hline 2012 & $\begin{array}{l}\text { Computer-based psychological treatments for depression: a systematic review and } \\
\text { meta-analysis. }\end{array}$ \\
\hline 2005 & A systematic review of guided imagery as an adjuvant cancer therapy. \\
\hline 2003 & Role conflict and academic procrastination: A self $\square$ determination perspective. \\
\hline 2019 & Analysis of smartphone addiction in university students. \\
\hline 1983 & $\begin{array}{l}\text { "Deep" RET: A reformulation of some psychodynamic explanations of } \\
\text { procrastination. }\end{array}$ \\
\hline 2018 & The internet addiction test in a young adult U.S. population. \\
\hline
\end{tabular}




\begin{tabular}{|l|l|}
1986 & $\begin{array}{l}\text { Affective, cognitive and behavioral differences between high and low } \\
\text { procrastinators. }\end{array}$ \\
\hline 1986 & $\begin{array}{l}\text { Affective, cognitive, and behavioral differences between high and low } \\
\text { procrastinators. }\end{array}$ \\
\hline 2013 & $\begin{array}{l}\text { Internet-based cognitive behavior therapy for procrastination: study protocol for a } \\
\text { randomized controlled trial. }\end{array}$ \\
\hline 2014 & $\begin{array}{l}\text { Understanding and treating procrastination: a review of a common self-regulatory } \\
\text { failure. }\end{array}$ \\
\hline 2015 & $\begin{array}{l}\text { Internet-based cognitive- behavior therapy for procrastination: a randomized } \\
\text { controlled trial. }\end{array}$ \\
\hline 2014 & $\begin{array}{l}\text { Group versus internet-based cognitive-behavioral therapy for procrastination: } \\
\text { study protocol for a randomized controlled trial. }\end{array}$ \\
\hline 2018 & $\begin{array}{l}\text { Social media use in lectures mediates the relationship between procrastination } \\
\text { and problematic smartphone use. }\end{array}$ \\
\hline 2010 & Structural equation models. \\
\hline 2019 & Future teachers' smartphone uses and dependence. \\
\hline 2017 & $\begin{array}{l}\text { Self-Determination Theory: Basic Psychological Needs in Motivation, } \\
\text { Development, and Wellness. }\end{array}$ \\
\hline 1999 & $\begin{array}{l}\text { Predictors of academic procrastination in college students. } \\
\text { satisfaction with life. }\end{array}$ \\
\hline 2002 & $\begin{array}{l}\text { Self continuity: Individual and collective perspectives. } \\
\text { school students who are academically at-risk. }\end{array}$ \\
\hline 2096 & Missing data: our view of the state of the art. \\
\hline
\end{tabular}




\begin{tabular}{|l|l|}
2006 & $\begin{array}{l}\text { New perspectives for the evaluation of training sessions in self-regulated } \\
\text { learning: time-series analyses of diary data. }\end{array}$ \\
\hline 1995 & Academic procrastination: Theoretical notions, measurement, and research. \\
\hline 2004 & $\begin{array}{l}\text { "Procrastination in academic settings: general introduction" in Counseling the } \\
\text { Procrastinator in Academic Settings }\end{array}$ \\
\hline 2001 & Study motivation under social temptation; effects of trait procrastination. \\
\hline 1995 & Trait procrastination and the big five factors of personality. \\
\hline 1995 & Trait procrastination and the big-five factors of personality. \\
\hline 2008 & $\begin{array}{l}\text { Modeling health behavior change: How to predict and modify the adoption and } \\
\text { maintenance of health behaviors. }\end{array}$ \\
\hline 1991 & $\begin{array}{l}\text { Self-handicapping by procrastinators: Protecting self-esteem, social-esteem, or } \\
\text { both. }\end{array}$ \\
\hline 1995 & Self-regulation and academic procrastination. \\
\hline 2003 & Role conflict and academic procrastination: a self-determination perspective. \\
\hline 1995 & $\begin{array}{l}\text { Prevalence of procrastination and the effects of cognitive-behavior and behavior } \\
\text { management therapies on reduction of procrastination in male \& female high } \\
\text { school students in Ahvaz. }\end{array}$ \\
\hline 1995 & $\begin{array}{l}\text { Self-regulation and academic procrastination. } \\
\text { directed framework. }\end{array}$ \\
\hline 2009 & $\begin{array}{l}\text { Assessing the psychometric properties of the Internet Addiction Test: A study on } \\
\text { a sample of Italian university students. }\end{array}$ \\
\hline 2002 & Intention-behavior relations: a conceptual and empirical review. \\
\hline
\end{tabular}




\begin{tabular}{|c|c|}
\hline 1984 & Imagery, physiology, and psychosomatic illness. \\
\hline 2015 & $\begin{array}{l}\text { Resilience and decision-making strategies as predictors of career decision } \\
\text { difficulties. }\end{array}$ \\
\hline 1981 & Procrastinating. \\
\hline 2001 & $\begin{array}{l}\text { New perspectives on autobiographical memory: The integration of narrative } \\
\text { processing and autobiographical reasoning. }\end{array}$ \\
\hline 2004 & $\begin{array}{l}\text { Procrastination and intentions to perform health behaviors: the role of self- } \\
\text { efficacy and the consideration of future consequences. }\end{array}$ \\
\hline 2014 & $\begin{array}{l}\text { Out of sight, out of time? A meta-analytic investigation of procrastination and } \\
\text { time perspective. }\end{array}$ \\
\hline 2014 & Procrastination and stress: exploring the role of self-compassion. \\
\hline 2003 & "I'll look after my health, later": an investigation of procrastination and health. \\
\hline 2013 & $\begin{array}{l}\text { Procrastination and the priority of short-term mood regulation: consequences for } \\
\text { future self. }\end{array}$ \\
\hline 2013 & $\begin{array}{l}\text { Procrastination and the priority of short-term mood regulation: consequences for } \\
\text { future self. }\end{array}$ \\
\hline 2015 & $\begin{array}{l}\text { Is procrastination a vulnerability factor for hypertension and cardiovascular } \\
\text { disease? Testing an extension of the procrastination-health model. }\end{array}$ \\
\hline 2014 & $\begin{array}{l}\text { Out of sight, out of time? A meta } \square \text { analytic investigation of procrastination and } \\
\text { time perspective. }\end{array}$ \\
\hline 2013 & $\begin{array}{l}\text { Procrastination and the priority of short } \square \text { term mood regulation: Consequences for } \\
\text { future self. }\end{array}$ \\
\hline 2012 & $\begin{array}{l}\text { Lost in the moment? An investigation of procrastination, mindfulness, and } \\
\text { well } \square \text { being. }\end{array}$ \\
\hline 2003 & "I'll look after my health, later": An investigation of procrastination and health. \\
\hline
\end{tabular}




\begin{tabular}{|l|l|}
1991 & Imagery in sport: An historical and current overview. \\
\hline 2005 & $\begin{array}{l}\text { Action planning and coping planning for long-term lifestyle change: theory and } \\
\text { assessment. }\end{array}$ \\
\hline 1984 & Academic procrastination: frequency and cognitive-behavioral correlates. \\
\hline 1984 & Academic procrastination: frequency and cognitive-behavioral correlates. \\
\hline 1984 & Academic procrastination: frequency and cognitive-behavioral correlates. \\
\hline 1984 & Academic procrastination: Frequency and cognitive-behavioral correlates. \\
\hline 2000 & Time orientations of procrastinators: Focusing on the past, present, or future? \\
\hline 2012 & Crossmodal mental imagery. \\
\hline 2018 & $\begin{array}{l}\text { Measurement invariance of the internet gaming disorder scale-short-form } \\
\text { (IGDS9-SF) between Australia, the USA, and the UK. }\end{array}$ \\
\hline 2010 & "I'll go to therapy, eventually": procrastination, stress and mental health. \\
\hline 2007 & $\begin{array}{l}\text { The nature of procrastination: a meta-analytic and theoretical review of } \\
\text { quintessential self-regulatory failure. }\end{array}$ \\
\hline 2007 & $\begin{array}{l}\text { The nature of procrastination: a meta-analytic and theoretical review of } \\
\text { quintessential self-regulatory failure. }\end{array}$ \\
\hline 2007 & $\begin{array}{l}\text { The Procrastination Equation: How to Stop Putting Things off and Start Getting } \\
\text { Stuff Done. }\end{array}$ \\
\hline $\begin{array}{l}\text { The nature of procrastination: A meta-analytic and theoretical review of } \\
\text { quintessential self-regulatory failure. }\end{array}$ \\
\hline 2001 & Procrastination and personality, performance, and mood. \\
\hline 2016 & Academic procrastination: psychological antecedents revisited. \\
\hline
\end{tabular}




\begin{tabular}{|c|c|}
\hline 2006 & Integrating theories of motivation. \\
\hline 2007 & $\begin{array}{l}\text { The nature of procrastination: A meta-analytic and theoretical review of } \\
\text { quintessential self-regulatory failure. }\end{array}$ \\
\hline 2007 & $\begin{array}{l}\text { The nature of procrastination: A meta-analytic and theoretical review of } \\
\text { quintessential self-regulatory failure. }\end{array}$ \\
\hline 2007 & $\begin{array}{l}\text { The nature of procrastination: a meta-analytic and theoretical review of } \\
\text { quintessential self regulatory failure. }\end{array}$ \\
\hline 2010 & Arousal, avoidant and decisional procrastinators: Do they exist? \\
\hline 2010 & $\begin{array}{l}\text { The procrastination equation: How to stop putting things off and start getting stuff } \\
\text { done. }\end{array}$ \\
\hline 2018 & $\begin{array}{l}\text { Examining procrastination across multiple goal stages: A longitudinal study of } \\
\text { temporal motivation theory. }\end{array}$ \\
\hline 2001 & $\begin{array}{l}\text { Worry, procrastination, and perfectionism: Differentiating amount of worry, } \\
\text { pathological worry, anxiety, and depression. }\end{array}$ \\
\hline 2015 & $\begin{array}{l}\text { Intervening to enhance proactivity in organizations: Improving the present or } \\
\text { changing the future. }\end{array}$ \\
\hline 2013 & $\begin{array}{l}\text { Development and validation of a } 2 \times 2 \text { model of time-related academic behavior: } \\
\text { procrastination and timely engagement. }\end{array}$ \\
\hline 2000 & $\begin{array}{l}\text { Subjective overachievement: Individual differences in self-doubt and concern } \\
\text { with performance. }\end{array}$ \\
\hline 2010 & Correlates and phenomenology of first and third person memories. \\
\hline 2006 & Using multivariate statistics. \\
\hline 2000 & $\begin{array}{l}\text { Task aversiveness and procrastination: A multi-dimensional approach to task } \\
\text { aversiveness across stages of personal projects. }\end{array}$ \\
\hline 2018 & $\begin{array}{l}\text { Prevalence rate of Internet addiction among Japanese college students: Two } \\
\text { cross-sectional studies and reconsideration of cut-off points of Young's Internet } \\
\text { Addiction Test in Japan. }\end{array}$ \\
\hline
\end{tabular}




\begin{tabular}{|c|c|}
\hline 1999 & The Big Five trait taxonomy: History, measurement, and theoretical perspectives. \\
\hline 1990 & The dimensions of perfectionism. Cognitive Therapy and Research. \\
\hline 1997 & The dispositional causes of job satisfaction: A core evaluations approach. \\
\hline 1999 & The effects of locus of control and task difficulty on procrastination. \\
\hline 2007 & $\begin{array}{l}\text { The evaluation of the major characteristics and aspects of the procrastination in } \\
\text { the framework of psychological counseling and guidance. }\end{array}$ \\
\hline 2007 & $\begin{array}{l}\text { The nature of procrastination: A meta-analytic and theoretical review of } \\
\text { quintessential self-regulatory failure. }\end{array}$ \\
\hline 2010 & $\begin{array}{l}\text { The role of general and performance self esteem in relation academic } \\
\text { procrastination and academic achievement. }\end{array}$ \\
\hline 2014 & $\begin{array}{l}\text { The roles of academic procrastination tendency on the relationships among self } \\
\text { doubt, self esteem and academic achievement. }\end{array}$ \\
\hline 2009 & The social construction of self-esteem, Oxford handbook of positive psychology. \\
\hline 2016 & The unintentional procrastination scale. \\
\hline 2019 & Comparison of internet addiction between teenagers and young adults. \\
\hline 2005 & Dispositional forgiveness of self, others, and situations. \\
\hline 1997 & $\begin{array}{l}\text { Longitudinal study of procrastination, performance, stress, and health: the costs } \\
\text { and benefits of dawdling. }\end{array}$ \\
\hline 2000 & $\begin{array}{l}\text { Giving in to feel good: the place of emotion regulation in the context of general } \\
\text { self-control. }\end{array}$ \\
\hline 2001 & $\begin{array}{l}\text { Emotional distress regulation takes precedence over impulse control: If you feel } \\
\text { bad, do it! }\end{array}$ \\
\hline 1997 & $\begin{array}{l}\text { Longitudinal study of procrastination, performance, stress, and health: the costs } \\
\text { and benefits of dawdling. }\end{array}$ \\
\hline
\end{tabular}




\begin{tabular}{|c|c|}
\hline 1997 & $\begin{array}{l}\text { Longitudinal study of procrastination, performance, stress, and health: The costs } \\
\text { and benefits of dawdling. }\end{array}$ \\
\hline 2000 & $\begin{array}{l}\text { Giving in to feel good: The place of emotion regulation in the context of general } \\
\text { self } \square \text { control. }\end{array}$ \\
\hline 2001 & $\begin{array}{l}\text { Emotional distress regulation takes precedence over impulse control: If you feel } \\
\text { bad, do it! }\end{array}$ \\
\hline 1995 & $\begin{array}{l}\text { Trait procrastination and the big-five factors of personality. Personality and } \\
\text { Individual Differences. }\end{array}$ \\
\hline 1991 & The development and concurrent validity of the procrastination scale. \\
\hline 1991 & The development and concurrent validity of the procrastination scale. \\
\hline 1999 & Underachieving to protect self-worth: Theory, research, and interventions. \\
\hline 1996 & $\begin{array}{l}\text { Patient non-compliance with drug regimens: measurement, clinical correlates, } \\
\text { economic impact. }\end{array}$ \\
\hline 2019 & Use and abuse of social media by adolescents: a study in Mexico. \\
\hline 2014 & $\begin{array}{l}\text { Habitus and flow in primary school musical pratice: relations between family } \\
\text { musical cultural capital, optimal experience and music paticipation. }\end{array}$ \\
\hline 2020 & $\begin{array}{l}\text { Young university students' academic self-regulation profiles and their associated } \\
\text { procrastination: autonomous functioning requires self-regulated operations. }\end{array}$ \\
\hline 2012 & $\begin{array}{l}\text { For fun, love, or money: What drives workaholic, engaged, and burned-out } \\
\text { employees at work? }\end{array}$ \\
\hline 2005 & Empathy gaps in emotional perspective taking. \\
\hline 2000 & Procrastination: self-regulation in initiating aversive goals. \\
\hline 2003 & A meta-analytically derived nomological network of procrastination. \\
\hline 2003 & A meta-analytically derived nomological network of procrastination. \\
\hline
\end{tabular}




\begin{tabular}{|c|c|}
\hline 2005 & $\begin{array}{l}\text { Bridging the gap between intentions and behavior: implementation intentions, } \\
\text { action control, and procrastination. }\end{array}$ \\
\hline 2007 & $\begin{array}{l}\text { Seeing future success: Does imagery perspective influence achievement } \\
\text { motivation? }\end{array}$ \\
\hline 2000 & $\begin{array}{l}\text { An examination of the content and construct validity of four measures of } \\
\text { procrastination (Unpublished master dissertation). }\end{array}$ \\
\hline 1997 & Relation between time structure and procrastination. \\
\hline 1998 & $\begin{array}{l}\text { Volitional action and inaction in the lives of undergraduate students: State } \\
\text { orientation, procrastination and proness to boredom. }\end{array}$ \\
\hline 1976 & Development and validation of the health locus of control (HLC) scale. \\
\hline 2011 & $\begin{array}{l}\text { Effects of group counseling based on self } \square \text { efficacy for self } \square \text { regulated learning in } \\
\text { students with academic procrastination. }\end{array}$ \\
\hline 2014 & $\begin{array}{l}\text { Effects of visual feedback on medical students' procrastination within web-based } \\
\text { planning and reflection protocols. }\end{array}$ \\
\hline 2001 & Procrastination and the five-factor model: a facet level analysis. \\
\hline 2001 & Procrastination and the five-factor model: A facet level analysis. \\
\hline 1977 & Imagery assessment: A survey of self $\square$ report measures. \\
\hline 2004 & The psychometric properties of the Internet addiction test. \\
\hline 2012 & Belonging to tomorrow: An overview of procrastination. \\
\hline 2010 & $\begin{array}{l}\text { I forgive myself, now I can study: how self-forgiveness for procrastinating can } \\
\text { reduce future procrastination. }\end{array}$ \\
\hline 2017 & Life course. \\
\hline 2001 & $\begin{array}{l}\text { Worry, procrastination, and perfectionism: Differentiating amount of worry, } \\
\text { pathological worry, anxiety and depression. }\end{array}$ \\
\hline
\end{tabular}




\begin{tabular}{|c|c|}
\hline 2019 & $\begin{array}{l}\text { An exploration of problematic smartphone use among Chinese university } \\
\text { students: Associations with academic anxiety, academic procrastination, self- } \\
\text { regulation and subjective wellbeing. }\end{array}$ \\
\hline 2016 & Validation of the short form of the academic procrastination scale. \\
\hline 2016 & $\begin{array}{l}\text { Internet addiction and relationships with insomnia, anxiety, depression, stress and } \\
\text { self-esteem in university students: a cross-sectional designed study. }\end{array}$ \\
\hline 1998 & $\begin{array}{l}\text { Caught in the net: How to recognize the signs of Internet addiction and a winning } \\
\text { strategy for recovery. }\end{array}$ \\
\hline 2016 & $\begin{array}{l}\text { Relationship of academic procrastination with metacognitive beliefs, emotion } \\
\text { regulation and tolerance of ambiguity in university students. }\end{array}$ \\
\hline 2015 & $\begin{array}{l}\text { How will I be remembered? Conserving the environment for the sake of one's } \\
\text { legacy. }\end{array}$ \\
\hline 2017 & $\begin{array}{l}\text { Frequency of Internet addiction and development of social skills in adolescents in } \\
\text { an urban area of Lima. }\end{array}$ \\
\hline 2018 & $\begin{array}{l}\text { Internet addiction, problematic Internet use, non problematic Internet use among } \\
\text { Chinese adolescents: Individual, parental, peer, and sociodemographic correlates. }\end{array}$ \\
\hline 2018 & $\begin{array}{l}\text { The development of academic procrastination in first-year secondary education } \\
\text { students: the link with metacognitive self-regulation, self-efficacy, and effort } \\
\text { regulation. }\end{array}$ \\
\hline 2007 & Book Reviews: Editor's Note: Guidelines for Selecting Books to Review \\
\hline 2013 & Book Reviews \\
\hline 2001 & The clause in English: In honour of Rodney Huddleston \\
\hline 2018 & $\begin{array}{l}\text { Is procrastination all that "bad"? A qualitative study of academic procrastination } \\
\text { and self-worth in postgraduate university students }\end{array}$ \\
\hline 2007 & $\begin{array}{l}\text { A Principal Components Analysis and Validation of the Coping with the College } \\
\text { Environment Scale (CWCES) }\end{array}$ \\
\hline
\end{tabular}




\begin{tabular}{|l|l|}
2019 & $\begin{array}{l}\text { Prospective memory (partially) mediates the link between ADHD symptoms and } \\
\text { procrastination }\end{array}$ \\
\hline 2009 & $\begin{array}{l}\text { Social Problem-Solving Processes and Mood in College Students: An } \\
\text { Examination of Self-report and Performance-based Approaches }\end{array}$ \\
\hline 1999 & Correspondence \\
\hline 2012 & $\begin{array}{l}\text { Developments in time preference and their implications for medical decision } \\
\text { making }\end{array}$ \\
\hline 2009 & Recent Studies in the Restoration and Eighteenth Century \\
\hline 2000 & $\begin{array}{l}\text { Staggering under the weight of responsibility: The impact of culture on physical } \\
\text { activity among African American women }\end{array}$ \\
\hline 2009 & $\begin{array}{l}\text { The Influence of Social Problem-Solving Ability on the Relationship Between } \\
\text { Daily Stress and Adjustment }\end{array}$ \\
\hline 1999 & $\begin{array}{l}\text { Drafting the International Criminal Court treaty: Two years to Rome and an } \\
\text { afterword on the Rome dimplomatic conference }\end{array}$ \\
\hline 2019 & Pre-crastination and procrastination effects occur in a reach-to-grasp task \\
\hline 2014 & Perfectionism and Motivation of Adolescents in Academic Contexts \\
\hline 2015 & $\begin{array}{l}\text { Academic procrastination and irrational thinking: A re-examination with context } \\
\text { controlled }\end{array}$ \\
\hline higher education
\end{tabular}




\begin{tabular}{|l|l|}
2017 & $\begin{array}{l}\text { Procrastinating Behavior in Computer-Based Learning Environments to Predict } \\
\text { Performance: A Case Study in Moodle }\end{array}$ \\
\hline 2016 & $\begin{array}{l}\text { Students' LMS interaction patterns and their relationship with achievement: A } \\
\text { case study in higher education }\end{array}$ \\
\hline 2009 & $\begin{array}{l}\text { Social Problem Solving as a Mediator of the Link Between Stress and } \\
\text { Psychological Well-being in Middle-Adulthood }\end{array}$ \\
\hline 2012 & $\begin{array}{l}\text { Co-Occurring Presenting Problems in African American College Clients } \\
\text { Reporting Racial Discrimination Distress }\end{array}$ \\
\hline 2000 & $\begin{array}{l}\text { Book Review: Facing the Decisions One Should Make and the Decisions One } \\
\text { Wants to Make }\end{array}$ \\
\hline 2019 & $\begin{array}{l}\text { Building word knowledge, learning strategies, and metacognition with the Word- } \\
\text { Knowledge e-Book }\end{array}$ \\
\hline 2017 & $\begin{array}{l}\text { Executive Functioning Among College Students With and Without History of } \\
\text { Childhood Maltreatment }\end{array}$ \\
\hline 1997 & The European convention on Bioethics \\
\hline 2020 & $\begin{array}{l}\text { Optimizing Students' Mental Health and Academic Performance: AI-Enhanced } \\
\text { Life Crafting }\end{array}$ \\
\hline 2008 & $\begin{array}{l}\text { Indecision and Avoidant Procrastination: The Role of Morningness-Eveningness } \\
\text { and Time Perspective in Chronic Delay Lifestyles }\end{array}$ \\
\hline How Do the Dimensions of Perfectionism Relate to Mental Health? \\
\hline 2000 & $\begin{array}{l}\text { Self-Critical Perfectionism, Coping, Hassles, and Current Distress: A Structural } \\
\text { Equation Modeling Approach } \\
\text { Unmasking Administrative Evil: The book and its critics / The authors' response }\end{array}$ \\
\hline 2996
\end{tabular}




\begin{tabular}{|l|l|}
2020 & $\begin{array}{l}\text { Instructional disobedience: a largely neglected phenomenon deserving more } \\
\text { systematic research attention }\end{array}$ \\
\hline 2003 & A CASE STUDY OF AN ACADEMIC RETENTION PROGRAM \\
\hline 1997 & Recent studies in tudor and stuart drama \\
\hline 2004 & Enough: THE FAILURE OF THE LIVING WILL \\
\hline 2018 & $\begin{array}{l}\text { Further development and testing of the metacognitive model of procrastination: } \\
\text { Self-reported academic performance }\end{array}$ \\
\hline 1998 & $\begin{array}{l}\text { The antecedents and consequences of academic excuse-making: Examining } \\
\text { individual differences in procrastination }\end{array}$ \\
\hline 2012 & $\begin{array}{l}\text { Procrastination Automatic Thoughts as a Personality Construct: An Analysis of } \\
\text { the Procrastinatory Cognitions Inventory }\end{array}$ \\
\hline 2016 & $\begin{array}{l}\text { Cognitive factors predicting checking, procrastination and other maladaptive } \\
\text { behaviours: Prospective versus Inhibitory Intolerance of Uncertainty }\end{array}$ \\
\hline 2002 & Remarks on introductory syntax \\
\hline 2019 & $\begin{array}{l}\text { Pilot study of a Web-based acceptance and commitment therapy intervention for } \\
\text { university students to reduce academic procrastination }\end{array}$ \\
\hline 2016 & $\begin{array}{l}\text { Treating procrastination chat-based versus face-to-face: An RCT evaluating the } \\
\text { role of self-disclosure and perceived counselor's characteristics }\end{array}$ \\
\hline and individual differences investigation \\
Academic Procrastination
\end{tabular}




\begin{tabular}{|c|c|}
\hline 1999 & An examination of the literature base on the suicidal behaviors of gifted students \\
\hline 2014 & $\begin{array}{l}\text { Avoiding procrastination through time management: an experimental intervention } \\
\text { study }\end{array}$ \\
\hline 2015 & $\begin{array}{l}\text { Decreasing students' stress through time management training: an intervention } \\
\text { study }\end{array}$ \\
\hline 2016 & $\begin{array}{l}\text { Procrastination is linked with insomnia symptoms: The moderating role of } \\
\text { morningness-eveningness }\end{array}$ \\
\hline 1999 & Parental affect and coping \\
\hline 2006 & $\begin{array}{l}\text { Frustration Intolerance Beliefs: Their Relationship with Depression, Anxiety, and } \\
\text { Anger, in a Clinical Population }\end{array}$ \\
\hline 2004 & $\begin{array}{l}\text { Problem-Solving Treatment for Complicated Depression in Late Life: A Case } \\
\text { Study in Primary Care }\end{array}$ \\
\hline 2018 & $\begin{array}{l}\text { Academic procrastination and feelings toward procrastination in LD and non-LD } \\
\text { students: Preliminary insights for future intervention }\end{array}$ \\
\hline 2010 & $\begin{array}{l}\text { Student contribution in asynchronous online discussion: a review of the research } \\
\text { and empirical exploration }\end{array}$ \\
\hline 2003 & $\begin{array}{l}\text { Differential Effect of Mirror Manipulation on Self-Perception in Social Phobia } \\
\text { Subtypes }\end{array}$ \\
\hline 2018 & Empower, not impose!-Preventing academic procrastination \\
\hline 2019 & $\begin{array}{l}\text { Acceptance and Commitment Training (ACT) as a Positive Psychological } \\
\text { Intervention: A Systematic Review and Initial Meta-analysis Regarding ACT's } \\
\text { Role in Well-Being Promotion Among University Students }\end{array}$ \\
\hline 2006 & $\begin{array}{l}\text { ACADEMIC, MOTIVATIONAL, AND EMOTIONAL PROBLEMS } \\
\text { IDENTIFIED BY COLLEGE STUDENTS IN ACADEMIC JEOPARDY }\end{array}$ \\
\hline 2000 & $\begin{array}{l}\text { A follow-up study of one group of achievers and underachivers four years after } \\
\text { high school graduation }\end{array}$ \\
\hline
\end{tabular}




\begin{tabular}{|c|c|}
\hline 1995 & $\begin{array}{l}\text { AN ANALYSIS OF THE CONTRIBUTION OF THE } 5 \text { FACTORS OF } \\
\text { PERSONALITY TO VARIANCE IN ACADEMIC PROCRASTINATION }\end{array}$ \\
\hline 2012 & $\begin{array}{l}\text { Libres Cahiers pour la Psychanalyse No. 21, Printemps 2010: L'angoisse [No. 21, } \\
\text { Spring 2010: Anxiety]1 }\end{array}$ \\
\hline 2019 & School-Related Risk Factors of Internet Use Disorders \\
\hline 2011 & $\begin{array}{l}\text { SUICIDE PRONENESS IN COLLEGE STUDENTS: RELATIONSHIPS WITH } \\
\text { GENDER, PROCRASTINATION, AND ACHIEVEMENT MOTIVATION }\end{array}$ \\
\hline 2013 & Procrastination in Different Life-Domains: Is Procrastination Domain Specific? \\
\hline 2013 & Procrastination When Good Things Don't Come to Those Who Wait \\
\hline 2017 & Rejection Sensitivity and Depression: Indirect Effects Through Problem Solving \\
\hline 2005 & $\begin{array}{l}\text { The Relationship of Motivation and Flow Experience to Academic } \\
\text { Procrastination in University Students }\end{array}$ \\
\hline 2019 & $\begin{array}{l}\text { Investigating and fostering self-regulated learning in higher education using } \\
\text { interactive ambulatory assessment }\end{array}$ \\
\hline 2019 & $\begin{array}{l}\text { Fostering self-regulation to overcome academic procrastination using interactive } \\
\text { ambulatory assessment }\end{array}$ \\
\hline 2018 & $\begin{array}{l}\text { Reducing procrastination using a smartphone-based treatment program: A } \\
\text { randomized controlled pilot study }\end{array}$ \\
\hline 2018 & Spotlight on math anxiety \\
\hline 2015 & Dispositional Optimism as a Correlate of Decision-Making Styles in Adolescence \\
\hline 1998 & $\begin{array}{l}\text { Performance management and welfare reform: The three-contingency model of } \\
\text { performance management applied to welfare reform }\end{array}$ \\
\hline 2019 & $\begin{array}{l}\text { I can't decide, and it upsets me: assessing self-critical cognition, indecision, and } \\
\text { hope among young adults }\end{array}$ \\
\hline 2019 & $\begin{array}{l}\text { The Multilevel Structure of Daily Emotion-Regulation-Strategy Use: An } \\
\text { Examination of Within- and Between-Person Associations in Naturalistic Settings }\end{array}$ \\
\hline
\end{tabular}




\begin{tabular}{|l|l|}
1998 & Women at midlife \\
\hline 2014 & $\begin{array}{l}\text { Psychostimulant use among college students during periods of high and low } \\
\text { stress: An interdisciplinary approach utilizing both self-report and unobtrusive } \\
\text { chemical sample data }\end{array}$ \\
\hline 2016 & $\begin{array}{l}\text { Social Media and Alcohol: Summary of Research, Intervention Ideas and Future } \\
\text { Study Directions }\end{array}$ \\
\hline 1998 & $\begin{array}{l}\text { Clarification of the conceptualization and measurement of denial in psychosocial } \\
\text { oncology research }\end{array}$ \\
\hline 2019 & Playing a video game is more than mere procrastination \\
\hline 2006 & Improved self-control: The benefits of a regular program of academic study \\
\hline 2018 & A Measure of Delay Discounting Within the Academic Domain \\
\hline 2018 & Developing an intervention to overcome procrastination \\
\hline 2013 & $\begin{array}{l}\text { Reducing Academic Procrastination Through a Group Treatment Program: A } \\
\text { Pilot Study }\end{array}$ \\
\hline 1998 & Contractual prayer of Christians and Muslims in Lebanon \\
\hline 2019 & $\begin{array}{l}\text { Inter-Rater Reliability of Cognitive-Behavioral Case Formulations of Depression: } \\
\text { A Replication }\end{array}$ \\
\hline A Meta-Analysis \\
\hline 2019 & $\begin{array}{l}\text { Psychological Counseling in Portuguese Higher Education: What are the } \\
\text { Second Edition }\end{array}$ \\
\hline Prevalents' Needs?
\end{tabular}




\begin{tabular}{|l|l|}
2016 & $\begin{array}{l}\text { Comprehending Poetry: What Literature Instructors Can Learn from Students' } \\
\text { Strategies }\end{array}$ \\
\hline 2000 & $\begin{array}{l}\text { Five days of emotion: An experience sampling study of undergraduate student } \\
\text { procrastination }\end{array}$ \\
\hline 2011 & $\begin{array}{l}\text { Academic procrastination in college students: The role of self-reported executive } \\
\text { function }\end{array}$ \\
\hline 2017 & How I Learned to Stop Worrying and Love Nudges \\
\hline 2018 & $\begin{array}{l}\text { Procrastination as a Self-Regulation Failure: The Role of Impulsivity and } \\
\text { Intrusive Thoughts }\end{array}$ \\
\hline 2018 & $\begin{array}{l}\text { Some Middle School Students Want Behavior Commitment Devices (but Take- } \\
\text { Up Does Not Affect Their Behavior) }\end{array}$ \\
\hline 2002 & Measures for Clinical Practice: A Sourcebook, 3rd ed \\
\hline 2001 & Adverb placement: A case study in antisymmetric syntax \\
\hline 2001 & Is the best good enough? Optimality and competition in syntax \\
\hline 2001 & Urban Jamaican Creole: Variation in the mesolect \\
\hline 2007 & $\begin{array}{l}\text { The Role of Experiential Avoidance in the Relationship Between Maladaptive } \\
\text { Perfectionism and Worry }\end{array}$ \\
\hline 2013 & $\begin{array}{l}\text { Reflexive violence } \\
\text { measurement and correlates of procrastination among school-aged children }\end{array}$ \\
\hline 2009 & Predictably Irrational: The Hidden Forces That Shape Our Decisions \\
\hline Measuring Learning in Depression Treatment: A Cognitive Bibliotherapy Test
\end{tabular}




\begin{tabular}{|c|c|}
\hline 2020 & $\begin{array}{l}\text { Piloting a New Model for Treating Music Performance Anxiety: Training a } \\
\text { Singing Teacher to Use Acceptance and Commitment Coaching With a Student }\end{array}$ \\
\hline 2001 & $\begin{array}{l}\text { Archaeology and language, vol. III: Artifacts, languages and texts / vol. IV: } \\
\text { Language change and cultural transformation }\end{array}$ \\
\hline 2019 & $\begin{array}{l}\text { COPD patients' experiences, self-reported needs, and needs-driven strategies to } \\
\text { cope with self-management }\end{array}$ \\
\hline 1999 & In memoriam: Mara Selvini-Palazzoli, M.D. (1946-1999) \\
\hline 2019 & $\begin{array}{l}\text { The Distinct Roles of Proximal and Distal Utility Values in Academic Behaviors: } \\
\text { Future Time Perspective as a Moderator }\end{array}$ \\
\hline 2003 & Cognitive Aspects of Compulsive Hoarding \\
\hline 1998 & Teacher-selected strategies for improving homework completion: RASE \\
\hline 2012 & $\begin{array}{l}\text { Understanding of and attitudes to academic ethics among first-year university } \\
\text { students }\end{array}$ \\
\hline 2006 & RESEARCH AND PRACTICE OF STUDENT RETENTION: WHAT NEXT? \\
\hline 2001 & Slips of the tongue: Speech errors in first and second language production \\
\hline 2018 & $\begin{array}{l}\text { A critical review of the literature on academic self-handicapping: theory, } \\
\text { manifestations, prevention and measurement }\end{array}$ \\
\hline 2020 & $\begin{array}{l}\text { Using mental contrasting with implementation intentions to reduce bedtime } \\
\text { procrastination: two randomised trials }\end{array}$ \\
\hline 2015 & Childhood attention-deficit/hyperactivity disorder \\
\hline 2017 & $\begin{array}{l}\text { A Field Experimental Design of a Strengths-Based Training to Overcome } \\
\text { Academic Procrastination: Short- and Long-Term Effect }\end{array}$ \\
\hline 2010 & Women With ADHD: It Is an Explanation, Not the Excuse Du Jour \\
\hline 1999 & Roosevelt and the Munich Crisis: A Study of Political Decision-Making \\
\hline
\end{tabular}




\begin{tabular}{|l|l|}
2018 & $\begin{array}{l}\text { Perfectionism as a Predictor of Anxiety in Foreign Language Classrooms among } \\
\text { Russian College Students }\end{array}$ \\
\hline 2000 & $\begin{array}{l}\text { Diabetes through the life span: Psychological ramifications for patients and } \\
\text { professionals }\end{array}$ \\
\hline 2014 & $\begin{array}{l}\text { Pharmacological interventions for adolescents and adults with ADHD: stimulant } \\
\text { and nonstimulant medications and misuse of prescription stimulants }\end{array}$ \\
\hline 2009 & Recent Studies in Tudor and Stuart Drama \\
\hline 2003 & $\begin{array}{l}\text { Of human bondage and literary triumphs: Hannah Crafts and the morphology of } \\
\text { the slave narrative }\end{array}$ \\
\hline 1999 & The emperor Mobutu \\
\hline 2019 & $\begin{array}{l}\text { Who is better adapted in learning online within the personal learning } \\
\text { environment? Relating gender differences in cognitive attention networks to } \\
\text { digital distraction }\end{array}$ \\
\hline 2007 & $\begin{array}{l}\text { Measuring Homework Utility in Psychotherapy: Cognitive-Behavioral Therapy } \\
\text { for Adult Attention-Deficit Hyperactivity Disorder as an Example }\end{array}$ \\
\hline 1998 & Direction of Fit and Normative Functionalism \\
\hline 2020 & $\begin{array}{l}\text { Effects of smartphone addiction on sleep quality among Chinese university } \\
\text { students: The mediating role of self-regulation and bedtime procrastination }\end{array}$ \\
\hline 2019 & $\begin{array}{l}\text { Self-efficacy for self-regulation and fear of failure as mediators between self- } \\
\text { esteem and academic procrastination among undergraduates in health professions }\end{array}$ \\
\hline The role of personality traits and need for cognition in active procrastination \\
\hline 2015 & APAGS Position Statement on the Rights of Psychology Graduate Students \\
\hline 2014 & APA/APAGS Statement on the Latest APPIC Internship Match \\
\hline 20 tuds
\end{tabular}




\begin{tabular}{|l|l|}
2010 & Doctoral Internships in Professional Psychology \\
\hline 2017 & Teaching Principles for Creative, Talented and Gifted Students \\
\hline 2008 & Recognized Specialties, Subspecialties and Proficiencies \\
\hline 2017 & GPE Grants-at-a-Glance \\
\hline 2017 & $\begin{array}{l}\text { Request for Applications: Building Research Infrastructure and Capacity Speaker } \\
\text { Series at Minority Serving Institutions }\end{array}$ \\
\hline 2015 & Practitioner Pointer: Supervising an intern or postdoctoral trainee \\
\hline 2018 & $\begin{array}{l}\text { Frequently Asked Questions: BEA Task Force to Develop a Blueprint for APA } \\
\text { Accreditation of Master's Programs in Health Service Psychology }\end{array}$ \\
\hline 2008 & Education and Training Resource Documents \\
\hline 2008 & APAGS elections and appointments \\
\hline 2018 & $\begin{array}{l}\text { WikiPizza: Increasing student engagement and learning about psychological } \\
\text { assessment }\end{array}$ \\
\hline 2011 & $\begin{array}{l}\text { Postdoctoral Education and Training Standards for The Specialty Practice of } \\
\text { Psychoanalysis in Psychology }\end{array}$ \\
\hline 2014 & Graduate Programs in Media Psychology \\
\hline 2013 & Students \\
\hline 2012 & Student and Career Information \\
\hline 52 Resolutions and Motions Regarding the Status of Women in Psychology: \\
\hline
\end{tabular}




\begin{tabular}{|l|l|}
2013 & Interviews with professionals in psychology and law \\
\hline 2012 & $\begin{array}{l}\text { Information on Undergraduate and Graduate Programs with Some Focus on } \\
\text { Religion }\end{array}$ \\
\hline 2018 & No Guns in Schools \\
\hline 2011 & Psychoanalytic-Friendly Graduate Programs in Psychoanalysis \\
\hline 2011 & Feminist Psychology Links \& Resources \\
\hline 2017 & 2017 Doctoral ARO Summary Data [PDF] \\
\hline 2018 & 2018 Doctoral ARO Summary Data \\
\hline 2013 & Resources for Teaching Psychology and Law \\
\hline 2013 & Education and Training in Psychology and Law \\
\hline n.d. & Overview of the Graduate Psychology Education (GPE) Program \\
\hline n.d. & $\begin{array}{l}\text { Guidelines for Reviewing Manuscripts for Training and Education in Professional } \\
\text { Psychology (TEPP) }\end{array}$ \\
\hline 2012 & Division of Adult Development and Aging Publications \\
\hline 2017 & 2017 Postdoctoral ARO Summary Data \\
\hline 2018 & 2018 Postdoctoral ARO Summary Data \\
\hline 2011 & Implementing Regulation C-20 Analysis: Internship Placement 2008-2010 \\
\hline 2018 & Div. 43 Postdoctoral Fellowship \\
\hline 2018 & Southern Region Internships \\
\hline 2018 & Midwest Region Internships \\
\hline 2018
\end{tabular}




\begin{tabular}{|l|l|}
2007 & $\begin{array}{l}\text { Council of Chairs of Training Councils (CCTC) Recommendations for } \\
\text { Communication }\end{array}$ \\
\hline 2014 & Webinar Series \\
\hline 2017 & Open Meeting Minutes 2017: RxP Designation Committee \\
\hline 2013 & Teaching Techniques \\
\hline 2017 & Welcome New Members \\
\hline 2018 & 2018 Internship ARO Summary Data \\
\hline 2017 & 2017 Internship ARO Summary Data \\
\hline 2008 & $\begin{array}{l}\text { The Impact of FERPA/HIPAA Regulations on Addressing Trainees with } \\
\text { Problems of Professional Competence (2008 Notes) }\end{array}$ \\
\hline 2017 & Teaching Quantitative and Qualitative Methods \\
\hline 2013 & Teaching Tips \\
\hline
\end{tabular}

